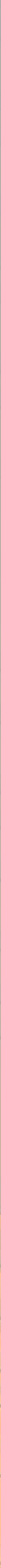




\title{
Fourier-Jacobi coefficients of Eisenstein series on unitary groups
}

\author{
Bei Zhang
}

This paper studies the Fourier-Jacobi expansions of Eisenstein series on $U(3,1)$. I relate the Fourier-Jacobi coefficients of the Eisenstein series with special values of $L$-functions. This relationship can be applied to verify the existence of certain Eisenstein series on $\mathrm{U}(3,1)$ that do not vanish modulo $p$. This is a crucial step towards one divisibility of the main conjecture for $\mathrm{GL}_{2} \times K^{\times}$using the method of Eisenstein congruences.

1. Introduction

283

2. Eisenstein series on $\mathrm{U}(3,1) \quad 288$

3. Theta functions 294

4. Fourier-Jacobi coefficients of Eisenstein series 303

5. Applications to Eisenstein series nonvanishing modulo $p \quad 324$

Appendix A. Proof of the Siegel-Weil formula for $(U(2,2), U(1)) 327$

Appendix B. Integral theta functions 332

Acknowledgments 336

References $\quad 336$

\section{Introduction}

Eisenstein congruence and Iwasawa main conjecture. Eisenstein series have been intensively used in constructions of $p$-adic $L$-functions and in the Iwasawa main conjectures. Ribet [1976] used the congruences between Eisenstein series and cusp forms to prove the converse of the Herbrand theorem. This idea was extended to congruences between $p$-adic families of modular forms, which was successfully used, first by Mazur and Wiles [1984] to prove the main conjecture for real abelian fields, then by Wiles [1990] for all totally real fields. Subsequently, Skinner and Urban used this technique to study the main conjectures for the motives attached to modular forms; see [Urban 2001; Urban 2006; Skinner and Urban 2012].

MSC2010: primary 11F55; secondary 11F30, 11F27, 11R23.

Keywords: Iwasawa main conjecture, unitary groups, Eisenstein series, Fourier-Jacobi expansion, doubling method, nonvanishing modulo $p$. 
In the ongoing joint project with Hsieh, we want to apply Eisenstein congruences to the following main conjecture for elliptic curves. Take an imaginary quadratic field $K$ in which $p$ splits. Fix an embedding $i_{\infty}: \overline{\mathbb{Q}} \rightarrow \mathbb{C}$ and an isomorphism $i: \mathbb{C} \cong \mathbb{C}_{p}$. Let

$$
\rho_{E}: \operatorname{Gal}(\overline{\mathbb{Q}} / \mathbb{Q}) \rightarrow \operatorname{GL}\left(H_{\mathrm{et}}^{1}\left(E, \mathbb{Q}_{p}\right)\right)
$$

be the $p$-adic Galois representation associated to an elliptic curve $E$ over $\mathbb{Q}$ with good ordinary reduction at $p$. Let $\eta: \mathbb{A}_{K}^{\times} / K^{\times} \rightarrow \mathbb{C}^{\times}$be a Hecke character with the infinity type $(k, 0)$, where $k$ is an integer and $k>1$. Put $0=\mathbb{Z}_{p}[\operatorname{Im} \eta]$, the ring of values of $\eta$. Let $K_{\max }$ be the unique $\mathbb{Z}_{p}^{2}$-extension of $K$, and let $\Gamma=\operatorname{Gal}\left(K_{\max } / K\right)$. Define $\Lambda=\mathcal{O} \llbracket \Gamma \rrbracket$ the Iwasawa algebra of $\Gamma$. Given $\Psi$ a $\Lambda$-valued character of $\operatorname{Gal}(\overline{\mathbb{Q}} / K)$ that interpolates $\eta$, there exists a unique element $L_{p}\left(\rho_{E} \otimes \Psi\right)$ in $\Lambda$ interpolating $\left((2 \pi i)^{2 k} / \Omega_{K}^{2 k}\right) L_{K}(0, E \otimes \eta)$, where $\Omega_{K}$ is the CM-period associated to $K$. (About the precise normalization of $L_{K}(E \otimes \eta, 0)$, one may check [Hsieh 2011 b, Definition 1]. Especially, since the weight of the CM form associated to $\eta$ is greater than 2, the period appearing in the denominator of the normalization of $L_{K}(E \otimes \eta, 0)$ is not the period of $E$, but the CM period attached to $\eta$ and $K$.) Let $\mathfrak{p}$ and $\overline{\mathfrak{p}}$ be the two primes in $K$ above $p$. Let $K_{S}$ be the maximal unramified extension of $K$ outside $S$, where

$$
S=\{\mathfrak{p}, \overline{\mathfrak{p}}\} \cup\left\{v \text { finite } \mid \eta_{v} \text { is ramified, or } E \text { has a bad reduction at } v\right\} \cup\{\infty\} \text {. }
$$

Use $M^{*}$ to denote the Pontryagin dual of a $\Lambda$-module $M$. Define the nonprimitive $\Lambda$-adic Selmer group to $\rho_{E} \otimes \Psi$ by

$$
\operatorname{Sel}_{K}\left(\rho_{E} \otimes \Psi\right)=\operatorname{ker}\left\{H^{1}\left(\operatorname{Gal}\left(K_{S} / K\right), T \otimes \Lambda^{*}\right) \rightarrow H^{1}\left(I_{\bar{p}}, T_{\overline{\mathfrak{p}}} \otimes \Lambda^{*}\right)\right\},
$$

where $T=H_{\mathrm{et}}^{1}\left(E, \mathbb{Z}_{p}\right), I_{\overline{\mathfrak{p}}}$ is the inertia group at $\overline{\mathfrak{p}} ;$ see [Hsieh $\left.2011 \mathrm{~b}\right]$. The following conjecture is formulated in [Greenberg 1994]:

Conjecture 1.1. $\operatorname{Sel}_{K}\left(\rho_{E} \otimes \Psi\right)$ is cotorsion over $\Lambda$, and for any height 1 prime $P$,

$$
\operatorname{ord}_{P} L_{p}\left(\rho_{E} \otimes \Psi\right)=l_{P}\left(\operatorname{Sel}_{K}\left(\rho_{E} \otimes \Psi\right)\right) \text {, }
$$

where $l_{P}\left(\operatorname{Sel}_{K}\left(\rho_{E} \otimes \Psi\right)\right)=\operatorname{length}_{\Lambda_{P}}\left(\operatorname{Sel}_{K}\left(\rho_{E} \otimes \Psi\right)^{*} \otimes_{\Lambda} \Lambda_{P}\right)$.

Remark 1.2. Though they have similar formulations, the conjecture above is different from the main conjecture of elliptic curves studied in [Skinner and Urban 2012], because the specializations of $\Psi$ are different. Our $\Psi$ interpolates Hecke characters over $K$ with the infinity type $(k, 0)$ for an integer $k>1$; the infinity types in [ibid.] are different. In addition, we consider different $L_{p}\left(\rho_{E} \otimes \Psi\right)$ and $\operatorname{Sel}_{K}\left(\rho_{E} \otimes \Psi\right)$. 
Nonvanishing modulo $p$ of Eisenstein series. Both for Ribet's original argument and for various cases of Iwasawa main conjectures, we crucially need to guarantee the Eisenstein series (or the $p$-adic Eisenstein series) used in the proofs does not vanish modulo $p$, for the naive reason that this is sufficient to deduce that the congruent cusp form is nontrivial. A more technical reason is that it is necessary to show that the constant term of this Eisenstein series divides the Eisenstein ideal, which is the first step towards one divisibility of the main conjecture.

For main conjectures of different cuspidal representations, Eisenstein series on different reductive groups are constructed so that the $p$-adic $L$-functions in the main conjectures interpolate the constant terms of the Eisenstein series. There is no consistent way to argue nonvanishing modulo $p$ of an Eisenstein series on a general reductive group. This question has been one of the obstacles of Iwasawa theory for $L$-functions of higher degrees. Urban [2006] argued that an Eisenstein-Klingen series on $\mathrm{GSp}(4)$ is nontrivial modulo $p$ in the following way: Find an algebraic linear combination of its Fourier coefficients (this is essentially a period integral of the Eisenstein series) that turns out to be a special $L$-value, and use Vatsal's result [2003] that this $L$-value does not vanish modulo $p$. A similar argument is used as well by Skinner and Urban [2012] to show an Eisenstein series on U(2,2) does not vanish modulo $p$.

To solve the main conjecture in our ongoing project, we need to construct an Eisenstein series on $\mathrm{U}(3,1)$. To attack the question about nonvanishing modulo $p$ of this Eisenstein series, the method is a bit different from other cases. Because $\mathrm{U}(3,1)$ is nonquasisplit, the Eisenstein series has a Fourier-Jacobi expansion where the coefficients are theta functions.

Results on Fourier-Jacobi coefficients. From now on, let $K$ be an imaginary quadratic extension of a totally real field $F$. Assume the degree of $F$ over $\mathbb{Q}$ is $r$. Let $P$ be the minimal parabolic subgroup of $\mathrm{U}(3,1)$ with $\mathrm{U}(2) \times K^{\times}$as the Levi part. Let $\Pi$ be an automorphic representation of $\mathrm{U}(2)(F) \backslash U(2)\left(\mathbb{A}_{F}\right)$, which corresponds to a holomorphic weight 2 cuspidal eigenform on $\mathrm{GL}_{2}(F)$. Take a Hecke character $\eta$ of $K^{\times}$such that $\eta_{\infty}\left(z_{\infty}\right)=\left|z_{\infty}\right|^{k} / z_{\infty}{ }^{k}$. The desirable Eisenstein series $E_{k}(\cdot, \Pi, \eta)$ of weight $k$ on $\mathrm{U}(3,1)$ is defined by pulling back a Siegel-Eisenstein series on $\mathrm{U}(3,3)$. This pullback idea was due to Shimura and was applied in the constructions of Eisenstein series in many cases; see [Urban 2006; Skinner and Urban 2012; Hsieh 2011b].

Let $\mathcal{M}_{k}(U(3,1))$ be the space of weight $k$ automorphic forms on $\mathrm{U}(3,1)$. A linear functional associated to a Bruhat-Schwartz function $\phi$ on $\mathbb{A}^{2}$ (where $\mathbb{A}$ is the adèle of $F$ ) can be defined by

$$
l_{\phi}: \mu_{k}(\mathrm{U}(3,1)) \rightarrow \mathbb{C}, \quad \mathbf{F} \mapsto \frac{\left\langle\mathbf{F}_{\psi}, \theta_{\phi}\right\rangle \cdot \theta_{\phi}^{\Pi}(1)}{\left\langle\theta_{\phi}, \theta_{\phi}\right\rangle},
$$


where $\theta_{\phi}^{\Pi}$ is the theta lifting from the cuspidal representation $\Pi$ on $\mathrm{U}(2)$ to $\mathrm{U}(1)$ defined by $\theta_{\phi}$, and $\mathbf{F}_{\psi}$ is the Fourier-Jacobi coefficient of $\mathbf{F}$ attached to the additive character $\psi$. When defining the theta lifting, a Hecke character $\chi$ will be introduced. So in the following Theorem 1.3, $\chi$ is implicit in the left side of the equation, but appears in the right. For the precise definition of the Fourier-Jacobi coefficient of $\mathbf{F}$, one can refer to $(25)$, and change $E(\cdot, f, s)$ in that equation to $\mathbf{F}$. Let $T_{\mathrm{A}}(\psi)$ be the space of adelic theta functions on $N(F) U(2)(F) \backslash N(\mathbb{A}) U(2)(\mathbb{A})$ with a well-defined inner product $\langle\cdot, \cdot\rangle$. Then $\mathbf{F}_{\psi}$ and $\theta_{\phi}$ are both elements inside.

In the following theorem, $S$ denotes the set of ramified places (the ramified places include places where any data used in the computation is ramified, for example, the characters $\eta$ and $\chi$ are ramified, the field extension $K / F$ is ramified, the representation $\Pi$ is ramified, and so on). We use $L^{S}$ to denote the partial $L$-function omitting the local $L$-factors at primes in $S$.

Theorem 1.3. For $E_{k}(\cdot, \Pi, \eta)$,

$$
\begin{aligned}
& l_{\phi}\left(E_{k}(\cdot, \Pi, \eta)\right) \\
& \quad=C \cdot \frac{(2 \pi i)^{(2 k-4) r} L^{S}\left(\eta \chi^{-1}, \frac{1}{2}(k-2)\right) L^{S}\left(\eta^{-1} \chi, \frac{1}{2}(k-4)\right) L^{S}(\Pi, \chi, 1)}{\Omega_{K}^{2 k}},
\end{aligned}
$$

where $C$ is a nonzero constant which can be explicitly calculated, $r=[F: \mathbb{Q}]$ and $\Omega_{K}$ is the CM-period associated to $K$.

The nature of $l_{\phi}\left(E_{k}(\cdot, \Pi, \eta)\right)$ depends on the normalization of $E_{k}(\cdot, \Pi, \eta)$. However, when we choose $E_{k}(\cdot, \Pi, \eta)$ to be rational, we can then show that $l_{\phi}\left(E_{k}(\cdot, \Pi, \eta)\right) \in \overline{\mathbb{Q}}$. So $C$ must be an algebraic number.

In the paper, the value of $l_{\phi}\left(E_{k}(\cdot, \Pi, \eta)\right)$ can simply be obtained by detailed computations of $\left\langle E_{k}(\cdot, \Pi, \eta), \theta_{\phi}\right\rangle$, from which one can prove Theorem 4.12. The proof uses computations of Fourier-Jacobi coefficients of the Siegel-Eisenstein series on $\mathrm{U}(3,3)$, whose definition can be found in (21); pullback formulas; the theta liftings for unitary groups; and the Siegel-Weil formula. By unfolding integrals step by step, the questions are translated to computations of Rallis inner product type. Then by studying the integral structure of the space of theta functions $T_{\mathbb{A}}(\psi)$, we can prove:

Proposition 1.4. For the p-integral holomorphic Eisenstein series $E_{k}, l_{\phi}\left(E_{k}\right)$ is a p-integer.

Conjecture 1.5. $E_{k}(\cdot, \Pi, \eta)$ does not vanish modulo $p$.

By the nonvanishing modulo $p$ of Hecke $L$-values (see [Hida 2004a]), we see that this conjecture is reduced to the question "Does there exist a Hecke character $\chi$ such that $L^{S}(\Pi, \chi, 1)$ does not vanish modulo $p$ ?" There have been many results 
of this flavor by Vatsal, Hida, Sun and Brakočević. I will consider this question in the near future.

The method introduced in this paper for calculating Fourier-Jacobi coefficients of Eisenstein series on $\mathrm{U}(3,1)$ can be generalized well to other nonquasisplit unitary groups. I will briefly explain the generalizations in Remark 5.7 at the end of this paper. For example, this method gives an alternative way to calculate the FourierJacobi coefficients of the Eisenstein series on $\mathrm{U}(2,1)$ other than the one given in [Murase and Sugano 2002], and recovers the proof of nonvanishing modulo $p$ of this Eisenstein series (compare to the discussion in [Mainardi 2004], which uses results in [Murase and Sugano 2002]).

The generalized computational results for Fourier-Jacobi coefficients of certain Eisenstein series on arbitrary unitary groups might inspire a new argument on the nonvanishing modulo $p$ of the Eisenstein series $E_{k}(\cdot, \Pi, \eta)$ on $\mathrm{U}(3,1)$. Briefly, one can construct an Eisenstein series on $\mathrm{U}(4,2)$ of the type explained in Remark 5.7. Thus the linear functional on this Eisenstein series will be the product of central special $L$-values, whose $p$-adic properties are much easier to study. Especially, we can first obtain nonvanishing modulo $p$ of this Eisenstein series. Then by studying the relations between Fourier-Jacobi coefficients of $E_{k}(\cdot, \Pi, \eta)$ on $\mathrm{U}(3,1)$ and this Eisenstein series on $\mathrm{U}(4,2)$, we can furthermore argue the nonvanishing modulo $p$ of $E_{k}(\cdot, \Pi, \eta)$. I hope to address this question soon in another paper.

Structure of the paper and some notation. Section 2 is about the theory of Eisenstein series on unitary groups. Two types of Eisenstein series are defined: one is on $\mathrm{U}(3,1)\left(\mathrm{U}\left(I_{\zeta}\right)\right)$, and the other is the Siegel type on $\mathrm{U}(3,3)$. Section $2 \mathrm{C}$ discusses the pullback formula. Theorem 2.6 gives the precise form, which is actually an adelic counterpart of "pullback of Eisenstein series" in [Shimura 1997].

Section 3 recalls the theory of Weil representations and theta functions. First, Section 3A introduces the Schrödinger representation $\rho_{\psi}$ of the Heisenberg group and the Weil representation $\omega_{\psi}$ of the metaplectic group. For this paper, we mainly need the Weil representation restricted to the dual reductive pair of unitary groups. The needed results are summarized in Section 3B, which also explains the relation between $\left.\omega_{\psi}\right|_{U(2)}$ and $\left.\omega_{\psi}\right|_{U(2,2)}$. Section $3 \mathrm{C}$ briefly recalls the theta lifting that appears in (1) and the Siegel-Weil formula, which is used to calculate the theta lifting.

Section 4 is about computations of Fourier-Jacobi coefficients of the two types of Eisenstein series used in the paper. The very important result that helps give a nice expression of the Fourier-Jacobi coefficients of $E_{k}(\cdot, \Pi, \eta)$ is summarized in Theorem 4.9.

Section 5, gives a strategic answer to the question of how to apply the results in Section 4 to show $\left.E_{k}(\cdot, \Pi, \eta)\right)$ on $\mathrm{U}(3,1)$ does not vanish modulo $p$. 
Unfortunately, the Siegel-Weil formula for the reductive pair $(\mathrm{U}(2), \mathrm{U}(1))$ that I use in this paper is not to be found in the literature - I give a proof in Appendix A. Appendix B gives a very brief discussion about integral theta functions, which is used in Section 5.

Let $G$ be an algebraic group defined over the totally real number field $F$. In this paper, we use $G(\mathbb{A})$ and $G(F)$ to denote the groups of adelic points and $F$-points of $G$, respectively. We use $[G]$ to denote the quotient $G(F) \backslash G(\mathbb{A})$. Given an arbitrary number field $L$ (for example, $\mathbb{Q}, F, K$ ), for each place $v$, we choose the additive Haar measure on $L_{v}$ so that, if $L_{v} \simeq \mathbb{R}$, the measure is the usual Lebesgue measure, if $L_{v} \simeq \mathbb{C}$, the measure is $2 d x d y(z=x+i y \in \mathbb{C})$, and if $v$ is a finite place, the measure gives the volume of the integer ring of $L_{v}$ to be $D_{L_{v}}^{-1 / 2}\left(D_{L_{v}}\right.$ is the absolute discriminant of $L_{v}$ ). The product of these local measures gives a measure on $\mathbb{A}_{L}$, and thus induces a measure on $L \backslash \mathbb{A}_{L}$. At each finite place $v$, the multiplicative measure on $L_{v}^{\times}$is taken such that $\operatorname{vol}\left(\mathcal{O}_{L_{v}}^{\times}\right)=1$, from which we obtain the measure on $\mathbb{A}_{L}^{\times}$. Notice that this multiplicative measure and the measure chosen in [Skinner and Urban 2012, 8.2.1] differ by a constant. The Haar measures on local and adelic points of algebraic groups should be clear from the definitions above and from the context.

\section{Eisenstein series on $U(3,1)$}

2A. Unitary groups. Let $K$ be a totally imaginary quadratic extension of a totally real number field $F$. Consider an $n$-dimensional $K$-vector space $V$ with an $\epsilon$-Hermitian form $\sigma$ for $\epsilon= \pm 1$. Without loss of generality, from now on we fix $\epsilon=-1$. The unitary group associated to $(V, \sigma)$ can be defined as follows:

$$
\mathrm{U}(\sigma)=\{g \in \mathrm{GL}(V) \mid \sigma(x g, y g)=(x, y) \text { for all } x, y \in V\} .
$$

To obtain a good matrix representation of $\mathrm{U}(\sigma)$, let us fix a Witt decomposition of $V$, with $V=J+Z+J^{\prime}$, where $J$ and $J^{\prime}$ are maximal totally $\sigma$-isotropic subspaces, and $Z$ is anisotropic or empty, so that $\operatorname{dim} J=\operatorname{dim} J^{\prime}=r$ and $\operatorname{dim} Z=t=n-2 r$. Under a suitable basis of $V$ consistent with the Witt decomposition, $\sigma$ can be expressed by the matrix

$$
I_{\zeta}=\left(\begin{array}{ccc}
0 & 0 & I_{r} \\
0 & \zeta & 0 \\
-I_{r} & 0 & 0
\end{array}\right)
$$

with $\zeta=-\zeta^{*} \in \mathrm{GL}_{t}(K)$. (From now on, we use $x^{*}$ to denote $\bar{x}^{t}$ for a matrix $x$.) Then:

$$
U(\sigma)=U\left(I_{\zeta}\right)=\left\{g \in \mathrm{GL}_{n}(K) \mid g I_{\zeta} g^{*}=I_{\zeta}\right\}
$$


Accordingly, the adelic unitary group $\mathrm{U}\left(I_{\zeta}\right)(\mathbb{A})$ and local groups $\mathrm{U}\left(I_{\zeta}\right)_{v}$ can be defined. Given a totally $\sigma$-isotropic subspace $J$ (which may not be maximal), put $P_{J}=\left\{g \in U\left(I_{\zeta}\right) \mid J g=J\right\}$. Then $P_{J}$ is the parabolic subgroup of $\mathrm{U}\left(I_{\zeta}\right)$ associated to $J$.

The Hermitian domain associated to $\mathrm{U}\left(I_{\zeta}\right)$ is defined as

$$
\mathfrak{Z}=\mathfrak{Z}(r, \zeta)=\left\{\left(\begin{array}{l}
x \\
y
\end{array}\right) \in \mathbb{C}_{r}^{r+t} \mid x \in \mathbb{C}_{r}^{r}, y \in \mathbb{C}_{r}^{t}, i\left(x^{*}-x\right)>i y^{*} \zeta^{-1} y\right\}
$$

Let

$$
g_{\infty}=\left(\begin{array}{lll}
a & b & c \\
d & e & f \\
h & l & p
\end{array}\right) \in U\left(I_{\zeta}\right)_{\infty}
$$

with $a, p \in \mathbb{C}_{r}^{r}$ and $e \in \mathbb{C}_{t}^{t}$. The action of $g_{\infty}$ on $\mathfrak{Z}(r, \zeta)$ is

$$
g_{\infty}\left(\begin{array}{l}
x \\
y
\end{array}\right)=\left(\begin{array}{c}
(a x+b y+c)(h x+l y+p)^{-1} \\
(d x+e y+f)(h x+l y+p)^{-1}
\end{array}\right) .
$$

The automorphy factor is

$$
j\left(g_{\infty},\left(\begin{array}{l}
x \\
y
\end{array}\right)\right)=\operatorname{det}(h x+l y+p) .
$$

Proposition 2.1. (1) Pick the origin $\boldsymbol{i}$ of $\mathfrak{Z}$ and put $C=\left\{g_{\infty} \in U\left(I_{\zeta}\right)_{\infty} \mid g_{\infty} \boldsymbol{i}=\boldsymbol{i}\right\}$. Then $C$ is a maximal compact subgroup of $\mathrm{U}\left(I_{\zeta}\right)_{\infty}$.

(2) Let $P=P_{J}$, where $J$ is a maximal totally isotropic subspace of $V$. Then $\mathrm{U}\left(I_{\zeta}\right)_{\infty}=P_{\infty} C$.

Assume $\operatorname{dim} V=4$ so that $J$ and $J^{\prime}$ are two isotropic lines, and $\mathrm{Z}$ is a 2dimensional anisotropic space; then $\mathrm{U}\left(I_{\zeta}\right) \subset \mathrm{GL}_{4}(K)$ is a degree 4 unitary group. From now on, whenever we write $\mathrm{U}\left(I_{\zeta}\right)$, I mean particularly this unitary group. To $\mathrm{U}\left(I_{\zeta}\right)$, there is only one nontrivial parabolic subgroup up to conjugation that is $P_{J}$. We can simply denote it by $P$. It consists of such elements

$$
p=\left(\begin{array}{ccc}
a & * & * \\
& u & * \\
& & \left(a^{*}\right)^{-1}
\end{array}\right),
$$

where $a \in K^{\times}$and

$$
u \in U(\zeta)=\left\{u \mid u \zeta u^{*}=\zeta\right\}
$$


2B. Eisenstein series. Following the notation above, $P$ is the parabolic subgroup of $\mathrm{U}\left(I_{\zeta}\right)$. It has the Levi decomposition $P=M \cdot N$, where $\mathrm{M}$ is the Levi part which is isomorphic to $\mathrm{U}(\zeta) \times \mathbb{G}_{m} / K$, and $N$ is the unipotent radical, consisting of elements like

$$
\left(\begin{array}{lll}
1 & * & * \\
& I_{2} & * \\
& & 1
\end{array}\right)
$$

Given a cuspidal representation $\Pi$ of $\mathrm{U}(\zeta)$ and a Hecke character $\eta$ of $K$, we get a cuspidal representation $\Pi \otimes \eta$ on the Levi part $M$. Then one has the induced representation

$$
I_{P}^{U\left(I_{\zeta}\right)}(\Pi \otimes \eta, s):=\operatorname{Ind}_{P(A)}^{U\left(I_{\zeta}\right)(A)} \delta_{P}^{\frac{1}{2}+s} \cdot \Pi \otimes \eta,
$$

where $\delta_{P}$ is the modulus character on $P$. If we denote by $V_{\Pi}$ the representation space of $\Pi$, then the representation space for $I_{P}^{U\left(I_{\zeta}\right)}(\Pi \otimes \eta, s)$ is the set of smooth functions $\tilde{f}_{s}: U\left(I_{\zeta}\right)(\mathbb{A}) \rightarrow V_{\Pi}$ such that

(1) $\tilde{f}_{s}(p g)=\delta_{P}(p)^{\frac{1}{2}+s} \cdot \Pi \otimes \eta(p)\left(\tilde{f}_{s}(g)\right), p \in P(\mathbb{A})$,

(2) $\tilde{f}_{s}$ is right $\mathbf{K}$-finite with $\mathbf{K}$ a maximal open compact subgroup of $\mathrm{U}\left(I_{\zeta}\right)(\mathbb{A})$.

The action of $\mathrm{U}\left(I_{\zeta}\right)(\mathbb{A})$ on $I_{P}^{U(3,1)}(\Pi \otimes \eta, s)$ is by right translation.

If we further assume that $\Pi$ is an irreducible submodule of $\mathscr{A}(M)$, where $\mathscr{A}(M)$ denotes the set of automorphic forms on $M$, we can realize $I_{P}^{U\left(I_{\zeta}\right)}(\Pi \otimes \eta, s)$ as $\mathbb{C}$-valued functions rather than functions valued in $V_{\Pi}$. For $\tilde{f}_{s} \in I_{P}^{U\left(I_{\zeta}\right)}(\Pi \otimes \eta, s)$, let $f_{s}(g)=\left(\tilde{f}_{s}(g)\right)(1)$. Then $f_{s}(g)$ satisfies $f_{s}(n m g)=\tilde{f}_{s}(g)(m)$. Define the Eisenstein series

$$
E(g, f, s)=\sum_{\gamma \in P(F) \backslash U\left(I_{\zeta}\right)(F)} f_{s}(\gamma g) .
$$

It can be proved that the sum converges absolutely when $\operatorname{Re}(s) \gg 0$, and can be continued to a meromorphic function on $\mathbb{C}$.

Aside from the Eisenstein series on $\mathrm{U}\left(I_{\zeta}\right)$, we also want to define an Eisenstein series on $U(3,3)$, which we are going to use in next section. Following definitions of unitary groups in the previous section,

$$
U(3,3)=\left\{g \in \mathrm{GL}_{6}(K) \mid g\left(\begin{array}{cc}
0 & I_{3} \\
-I_{3} & 0
\end{array}\right) g^{*}=\left(\begin{array}{cc}
0 & I_{3} \\
-I_{3} & 0
\end{array}\right)\right\} .
$$

The Siegel parabolic subgroup $\mathscr{P}$ of $\mathrm{U}(3,3)$ is the one that fixes the maximal totally isotropic space of dimension 3 in a 6-dimensional Hermitian vector space. The Levi part of $\mathscr{P}$ is isomorphic to $\mathrm{GL}_{3}(K)$. Take the one-dimensional representation 
$\eta($ det $\cdot)$ on the Levi part. Then it induces the representation on $\mathrm{U}(3,3)$ as

$$
I_{\mathscr{P}}^{U(3,3)}(\eta, s):=\operatorname{Ind}_{\mathscr{P}(\mathrm{A})}^{U(3,3)(\mathrm{A})} \delta_{\mathscr{P}}^{\frac{1}{2}+s} \cdot \eta(\mathrm{det} \cdot) .
$$

Pick a section $\mathbb{E}_{s}$ in the induced representation. The Siegel-Eisenstein series is defined by

$$
\mathbb{E}(g, \mathbb{E}, s)=\sum_{\gamma \in \mathscr{P}(F) \backslash U(3,3)(F)} \mathbb{\mathbb { N }}_{s}(\gamma g) \quad \text { for } g \in U(3,3)(\mathbb{A}) .
$$

It satisfies analytic properties similar to $E(\cdot, f, s)$.

2C. Pullback formulas. In this section, the Eisenstein series $E(g, f, s)$ on $\mathrm{U}\left(I_{\zeta}\right)$ is constructed using the pullback of a Siegel Eisenstein series on $U(3,3)$.

The unitary group $\mathrm{U}(\zeta)$ is closely related to the quaternion algebra, about which, let me quote two lemmas from [Shimura 1997].

Lemma 2.2. $V$ is a 2-dimensional $K$-vector space with a nondegenerate Hermitian pairing described by $\zeta$. Then $V$ is anisotropic if and only if $\operatorname{det} \zeta$ is represented by -1 in $K^{\times} / N_{K / F}\left(K^{\times}\right)$.

Lemma 2.3. Let $(V, \zeta)$ be anisotropic.

(1) $D:=\left\{\alpha \in \operatorname{End}(V) \mid \sigma\left(\alpha^{l} x, y\right)=\sigma(x, \alpha y)\right.$ for all $\left.x, y \in V\right\}$, where เ is the main involution of $\operatorname{End}(V)$ such that $\left(\begin{array}{ll}a & b \\ c & d\end{array}\right)^{l}=\left(\begin{array}{cc}d & -b \\ -c & a\end{array}\right)$. Then $D$ is a definite quaternion algebra over $F$.

(2) $\mathrm{U}(\zeta)=\left\{s \alpha \mid s \in K^{\times}, \alpha \in D^{\times}, s s^{\rho} \operatorname{det} \alpha=1\right\}$.

So, locally:

(1) When $K_{v}$ splits, namely $K_{v}=F_{v} \times F_{v}$, we have $\mathrm{U}(\zeta)_{v} \simeq \mathrm{GL}_{2}\left(F_{v}\right)$.

(2) When $K_{v}$ is a field and $d\left(\sigma_{v}\right)$ is represented by 1 , we have $\mathrm{U}(\zeta)_{v}=U(1,1)_{v}$.

(3) When $K_{v}$ is a field and $d\left(\sigma_{v}\right)$ is represented by -1 (including archimedean places), we have $\mathrm{U}(\zeta)_{v} \subset K_{v}^{\times} \cdot D_{v}^{\times}$, and $D_{v}$ is a quaternion algebra. In this case, $\mathrm{U}(\zeta)_{v}$ is compact.

The following theorem about the Jacquet-Langlands correspondence relates automorphic forms on $D^{\times}$to automorphic forms on $\mathrm{GL}_{2}(F)$.

Theorem 2.4 [Gelbart 1975]. $S$ is the set of places of $F$ where $D$ is ramified. To each irreducible unitary admissible representation $\pi=\otimes_{v} \pi_{v}$ of $D_{A}^{\times}$, let $\pi^{\prime}$ denote the representation of $\mathrm{GL}_{2}(\mathbb{A})$ whose $v$-th component is equivalent to $\pi_{v}$ if $v \notin S$, and special or supercuspidal if $v \in S$.

(1) The map $\pi \rightarrow \pi^{\prime}$ restricted to the collection of (greater than one-dimensional) cusp forms on $D^{\times}$is one-to-one onto the collection of all (equivalence classes of) cusp forms on $\mathrm{GL}_{2}(F)$ such that $\pi_{v}^{\prime}$ is square-integrable for each $v \in S$. 
(2) If we require further that $\pi \subset L^{2}\left(D^{\times}(F) \backslash D^{\times}(\mathbb{A})\right)$, then it implies that $\pi_{v}^{\prime}$ is one-dimensional for $v \in S$.

For an irreducible representation $\pi$ on the definite quaternion algebra $D, \pi_{\infty}$ as a finite-dimensional representation of $D_{\infty}^{\times}$is equivalent to $|\operatorname{det}|^{r} \otimes \rho_{n}$, where $\rho_{n}$ is the $n$-th symmetric tensor product. It corresponds to the discrete series $\sigma\left(\mu_{1}, \mu_{2}\right)$ of $\mathrm{GL}_{2}(\mathbb{R}) \quad$ with $\mu_{1}(x)=|x|^{r+n+\frac{1}{2}} \quad$ and $\quad \mu_{2}(x)=|x|^{r-\frac{1}{2}} \operatorname{sgn}(x)^{n}$.

Globally, $\pi$ corresponds to a cuspidal newform $f$ that is also a Hecke eigenform of weight $k=n+2$.

Take a representation $\Pi$ of $\mathrm{U}(\zeta)$ in this way: First pick an irreducible representation $\lambda \cdot \pi$ on $\mathbb{A}_{K}^{\times} \cdot D_{\mathbb{A}}^{\times}$satisfying $\left.\lambda\right|_{\mathbb{A}^{\times}}=\chi_{\pi}$, where $\chi_{\pi}$ is the central character of $\pi$. Then $\Pi=\left.(\lambda \cdot \pi)\right|_{U(\zeta)(\mathbb{A})}$ gives an irreducible representation on $\mathrm{U}(\zeta)$. For our later application, the cusp form $f$ that $\pi$ corresponds to comes from an elliptic curve. So $f$ has weight $k=2$. Then $\Pi_{\infty}$ must be one-dimensional. In this case, the pullback formula will have a simple form. So in this paper, we always assume $\Pi$ satisfies this condition.

Define an embedding

$$
\mathfrak{e}: U\left(I_{\zeta}\right) \times U(\zeta) \rightarrow U(3,3), \quad(g, u) \mapsto \mathfrak{e}(g, u)=A_{\zeta}\left({ }^{g}{ }_{u}\right) A_{\zeta}^{-1},
$$

where

$$
A_{\zeta}=\left(\begin{array}{cccc}
1 & & & \\
& \zeta^{-1} & & -\zeta^{-1} \\
& & 1 & \\
& \frac{1}{2} & & \frac{1}{2}
\end{array}\right) .
$$

Pay attention that $\mathrm{U}(\zeta)$ has no nontrivial parabolic subgroup. If $\mathscr{P}$ is the Siegel parabolic subgroup of $\mathrm{U}(3,3)$ and $P$ is the only nontrivial parabolic subgroup of $\mathrm{U}\left(I_{\zeta}\right)$, we have the following lemma:

Lemma 2.5 [Shimura 1997]. $\mathscr{P}(F) \backslash U(3,3)(F)=\mathfrak{e}\left(P(F) \backslash U\left(I_{\zeta}\right)(F), U(\zeta)(F)\right)$.

Take $\boldsymbol{\bigcirc}_{s} \in I_{\mathscr{P}}^{U(3,3)}(\eta, s)$. Define the Eisenstein series $\mathbb{E}(g, \mathbb{E}, s)$ on $\mathrm{U}(3,3)$ by (7). Theorem 2.6. Take $\beta \in V_{\Pi} \subset L^{2}(U(\zeta)(F) \backslash U(\zeta)(\mathbb{A}))$. Then the integral

$$
\int_{U(\zeta)(F) \backslash U(\zeta)(\mathbb{A})} \mathbb{E}(\mathfrak{e}(g, u), \mathbb{f}, s) \beta(u) \eta^{-1}(\operatorname{det} u) d u
$$

gives an Eisenstein series $E(g, f, s)$ on $\mathrm{U}\left(I_{\zeta}\right)$ associated to $f_{s} \in I_{P}^{U\left(I_{\zeta}\right)}(\Pi \otimes \eta, s)$, and

$$
f_{s}(g)=\int_{U(\zeta)(\mathbb{A})} \mathbb{E}_{s}(\mathfrak{e}(g, u)) \beta(u) \eta^{-1}(\operatorname{det} u) d u .
$$

Here we understand $\beta(u)$ as $(\Pi(u) \beta)(1)$. 
Proof. General results about the pullback formula at infinity are essentially discussed in [Shimura 1997]. Here I give a proof using the adelic language. Using Lemma 2.5,

$$
\begin{aligned}
\mathbb{E}(\mathfrak{e}(g, u), \mathbb{F}, s) & =\sum_{\gamma \in \mathscr{P}(F) \backslash U(3,3)(F)} \mathbb{E}_{s}(\gamma \mathfrak{e}(g, u)) \\
& =\sum_{\gamma_{1} \in P(F) \backslash U\left(I_{\zeta}\right)(F)} \sum_{\gamma_{2} \in U(\zeta)(F)} \mathbb{a}_{s}\left(\mathfrak{e}\left(\gamma_{1} g, \gamma_{2} u\right)\right),
\end{aligned}
$$

unfold the integral:

$$
\begin{aligned}
\int_{U(\zeta)(F) \backslash U(\zeta)(\mathbb{A})} & \mathbb{E}(\mathfrak{e}(g, u), \mathbb{F}, s) \beta(u) \eta^{-1}(\operatorname{det} u) d u \\
= & \int_{U(\zeta)(F) \backslash U(\zeta)(\mathbb{A})} \sum_{\gamma_{1} \in P(F) \backslash U\left(I_{\zeta}\right)(F)} \sum_{\gamma_{2} \in U(\zeta)(F)} \mathbb{E}_{s}\left(\mathfrak{e}\left(\gamma_{1} g, \gamma_{2} u\right)\right) d u \\
= & \int_{U(\zeta)(\mathbb{A})} \sum_{\gamma_{1} \in P(F) \backslash U\left(I_{\zeta}\right)(F)} \mathbb{E}_{s}\left(\mathfrak{e}\left(\gamma_{1} g, u\right)\right) \beta(u) \eta^{-1}(\operatorname{det} u) d u \\
= & \sum_{\gamma_{1} \in P(F) \backslash U(3,1)(F)} \int_{U(\zeta)(\mathbb{A})} \mathbb{E}_{s}\left(\mathfrak{e}\left(\gamma_{1} g, u\right)\right) \beta(u) \eta^{-1}(\operatorname{det} u) d u .
\end{aligned}
$$

In the last step, we suppose $s$ is in a proper range so that there are no convergence problems. Then we can change the order of the integral and the summation. Formally, (*) looks like the Eisenstein series $E(g, f, s)$ on $\mathrm{U}\left(I_{\zeta}\right)$ defined by (5) with

$$
f_{s}=\int_{U(\zeta)(\mathbb{A})} \mathbb{F}_{s}(\mathfrak{e}(g, u)) \beta(u) \eta^{-1}(\operatorname{det} u) d u
$$

We are left to show that $f_{s} \in I_{P}^{U\left(I_{\zeta}\right)}(\Pi \otimes \eta, s)$. Take

$$
p=\left(\begin{array}{ccc}
a & * & * \\
& v & * \\
& & \left(a^{*}\right)^{-1}
\end{array}\right) \in P(\mathbb{A}),
$$

and also note that

$$
\mathfrak{e}\left(\left(\begin{array}{ccc}
a & * & * \\
& v & * \\
& \left(a^{*}\right)^{-1}
\end{array}\right), v\right)=\left(\begin{array}{cccc}
a & * & * & * \\
& \zeta^{-1} v \zeta & * & * \\
& & \left(a^{*}\right)^{-1} & * \\
& & & v
\end{array}\right) \in \mathscr{P}(\mathbb{A})
$$


by the embedding formula (8). So

$$
\begin{aligned}
& f_{s}(p g)=\int_{U(\zeta)(\mathbb{A})} \mathbb{E}_{s}(\mathfrak{e}(p g, u)) \beta(u) \eta^{-1}(\operatorname{det} u) d u \\
& =\int_{U(\zeta)(\mathbb{A})} \mathbb{E}_{s}(\mathfrak{e}(p g, v u)) \beta(v u) \eta^{-1}(\operatorname{det} v u) d u \\
& =\int_{U(\zeta)(\mathbb{A})} \mathbb{E}_{s}\left(\mathfrak{e}\left(\left(\begin{array}{ccc}
a & * & * \\
& v & * \\
& & \left(a^{*}\right)^{-1}
\end{array}\right), v\right) \cdot \mathfrak{e}(g, u)\right) \beta(v u) \eta^{-1}(\operatorname{det} v u) d u
\end{aligned}
$$

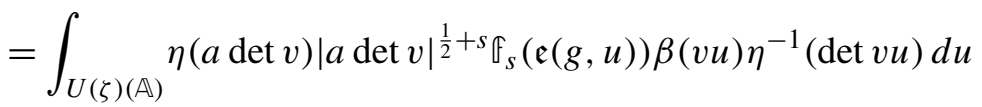

$$
\begin{aligned}
& =\Pi \otimes \eta(p) f_{s}(g)|a|^{\frac{1}{2}+s} \text {. }
\end{aligned}
$$

This means that $f_{s} \in I_{P}^{U\left(I_{\zeta}\right)}(\Pi \otimes \eta, s)$.

\section{Theta functions}

3A. Weil representations. Suppose $V$ is a finite-dimensional vector space over a field $\mathbb{F}$. When $\mathbb{F}$ is a nonarchimedean local field, use $S(V)$ to denote the space of Bruhat-Schwartz functions (locally constant compactly supported functions) on $V$. If $F=\mathbb{R}$ or $\mathbb{C}$, we first take $S(V)$ to be $L^{2}(V)$ (but later, we may add holomorphic conditions when needed).

Following the notation in previous sections, $F$ is a totally real number field. Let $W$ be a finite-dimensional symplectic vector space over $F_{v}$ with a nondegenerate alternating form $\langle\cdot, \cdot\rangle$. The Heisenberg group $H(W)$ associated to $W$ is a nontrivial central extension of $W$ by $F_{v}$ and is defined to be a group of pairs $\{(w, t) \mid w \in$ $\left.W, t \in F_{v}\right\}$ with the law of multiplication

$$
\left(w_{1}, t_{1}\right)\left(w_{2}, t_{2}\right)=\left(w_{1}+w_{2}, t_{1}+t_{2}+\frac{1}{2}\left\langle w_{1}, w_{2}\right\rangle\right) .
$$

Fix an additive character $\psi$ of $F_{v}$ and a complete polarization of $W$ as $W=X \oplus Y$ where $X$ and $Y$ are maximal totally isotropic subspaces of $W$. Define the Schrödinger representation $\rho_{\psi}$ of $H(W)$ on $S(X)$ as follows:

$$
\begin{array}{ll}
\rho_{\psi}(x) f(z)=f(x+z) & \text { for } x \in X, \\
\rho_{\psi}(y) f(z)=\psi(\langle z, y\rangle) f(z) & \text { for } y \in Y, \\
\rho_{\psi}(t) f(z)=\psi(t) f(z) & \text { for } t \in F_{v} .
\end{array}
$$

Theorem 3.1 (Stone, von Neumann). $H(W)$ has a unique irreducible smooth representation on which $F_{v}$ operates via the character $\psi$. 
One may have seen other constructions of smooth irreducible representations of $H(W)$ on which the center acts by the character $\psi$. However, by Theorem 3.1, they are isomorphic to one another. In this paper, I only use $\left(\rho_{\psi}, S(X)\right)$ defined above, which is so-called the Schrödinger model.

The symplectic group $\mathrm{Sp}(W)$ has an action on $H(W)$ as $g \cdot(w, t)=(g w, t)$. By the uniqueness theorem of Stone and von Neumann, there is an operator $\omega_{\psi}(g)$ on $S(X)$ that it is unique up to scalar and satisfies

$$
\rho_{\psi}(g w, t) \omega_{\psi}(g)=\omega_{\psi}(g) \rho_{\psi}(w, t) \quad \text { for all }(w, t) \in H(W) .
$$

Define the metaplectic group

$$
\widetilde{\mathrm{Sp}}_{\psi}(W)=\left\{\left(g, \omega_{\psi}(g)\right) \text { such that }(10) \text { holds }\right\}
$$

which fits in the following exact sequence:

$$
1 \rightarrow \mathbb{C}^{\times} \rightarrow{\widetilde{\mathrm{Sp}_{\psi}}}(W) \stackrel{\text { proj }}{\longrightarrow} \mathrm{Sp}(W) \rightarrow 1
$$

The Weil representation of $\tilde{\mathrm{Sp}}_{\psi}(W)$ is the one obtained by projecting to the second factor $\left(g, \omega_{\psi}(g)\right) \rightarrow \omega_{\psi}(g)$. Under the Schrödinger model $\left(\rho_{\psi}, S(X)\right)$, the Weil representation can be explicitly written down:

$$
\begin{aligned}
\omega_{\psi}\left(\begin{array}{ll}
A & \\
& \left(A^{t}\right)^{-1}
\end{array}\right) \phi(x) & =|\operatorname{det} A|^{\frac{1}{2}} \phi(x A), \\
\omega_{\psi}\left(\begin{array}{rr}
I & B \\
I
\end{array}\right) \phi(x) & =\psi\left(\frac{x B x^{t}}{2}\right) \phi(x), \\
\omega_{\psi}\left(\begin{array}{rr}
I \\
-I
\end{array}\right) \phi(x) & =\gamma \hat{\phi}(x),
\end{aligned}
$$

where $\phi \in S(X), \hat{\phi}$ is the Fourier transform

$$
\hat{\phi}(x)=\int_{F_{v}^{n}} \phi(y) \psi\left(\sum_{i=1}^{n} x_{i} y_{i}\right) d y,
$$

the Haar measure $d y$ is chosen so that $\hat{\hat{\phi}}=\phi(-x)$, and $\gamma$ is an 8-th root of unity determined by $\psi$.

The discussion above in the local case can mostly be generalized to the global case. The global Schrödinger representation $\rho_{\psi}$ of $H(W)(\mathbb{A})$ on $S(X(\mathbb{A}))$ can be defined, where

$$
S(X(\mathbb{A}))=\left\{\otimes_{v} \phi_{v} \mid \phi_{\infty} \in L^{2}\left(X_{\infty}\right), \phi_{v} \in S\left(X_{v}\right) \text {, and for a.a. } v, \hat{\phi}_{v}=\phi_{v}\right\} .
$$


Also we have the global Weil representation $\omega_{\psi}$ of $\widetilde{\mathrm{Sp}}(W)(\mathbb{A})$ on $S(X(\mathbb{A}))$, and for each place $v$ of $F$, one has the following commutative diagram:

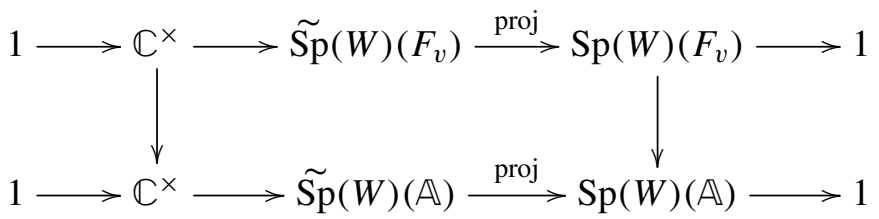

\section{B. Dual reductive pair.}

Definition. A dual reductive pair is a pair of subgroups $\left(G, G^{\prime}\right)$ of the symplectic group $\mathrm{Sp}(W)$ such that

(1) $G$ is the centralizer of $G^{\prime}$ in $\operatorname{Sp}(W)$ and vice versa, and

(2) the actions of $G$ and $G^{\prime}$ are completely reducible on $W$. (An action is called completely reducible if every invariant subspace has an invariant complement.)

Obviously this definition can be applied locally or globally.

In this paper, the types of reductive pairs used are the following: $K$ is again an imaginary quadratic extension of $F,\left(V_{1},(\cdot, \cdot)_{1}\right)$ is a skew-Hermitian space over $K$, and $\left(V_{2},(\cdot, \cdot)_{2}\right)$ is a Hermitian space over $K$. Take $W=V_{1} \otimes V_{2}$, and on $W$ define an alternating form $\frac{1}{2} \operatorname{tr}_{K / F}(\cdot, \cdot)_{1} \otimes{\overline{(\cdot, \cdot)_{2}}}_{2}$. Then the unitary groups $\mathrm{U}\left(V_{1}\right)$ and $\mathrm{U}\left(V_{2}\right)$ form a dual reductive pair in $\operatorname{Sp}(W)$. We have the embedding

$$
e: U\left(V_{1}\right) \times U\left(V_{2}\right) \rightarrow \operatorname{Sp}(W), \quad\left(g_{1}, g_{2}\right) \cdot\left(v_{1} \otimes v_{2}\right) \mapsto=v_{1} g_{1} \otimes g_{2}^{-1} v_{2} .
$$

In this paper, we use $e$ to denote the embeddings of unitary groups into symplectic groups, and use $\mathfrak{e}$ to denote the embeddings of the same type of groups, such as the embedding of one unitary group into another unitary group, or the embedding of one symplectic group into another symplectic group.

Splittings. For a fixed Weil representation $g \rightarrow \omega(g)$ (for example, the Weil representation $\omega_{\psi}$ defined through the Schrödinger model), we can define $c\left(g_{1}, g_{2}\right)$ so that

$$
\omega\left(g_{1}\right) \omega\left(g_{2}\right)=c\left(g_{1}, g_{2}\right) \omega\left(g_{1} g_{2}\right) .
$$

Then $c: \operatorname{Sp}(W) \times \mathrm{Sp}(W) \rightarrow \mathbb{C}^{\times}$is a 2-cocycle whose class is in the cohomology group $H^{2}\left(\operatorname{Sp}(W), \mathbb{C}^{\times}\right)$. When the additive character $\psi$ of $F$ and one maximal isotropic subspace of $W$ are fixed, $c$ is determined using the Leray invariant. The following proposition claims that under certain condition, $c$ could be a coboundary. Returning to the dual reductive pair $\left.\left(U\left(V_{1}\right), U\left(V_{2}\right)\right)\right)$ of $\operatorname{Sp}(W)$, we have this:

Proposition 3.2 [Harris et al. 1996]. $\widetilde{\mathrm{Sp}}(W)$ splits over $\mathrm{U}\left(V_{i}\right)$ compatibly with respect to rational points for $i=1,2$. In particular, there is a splitting homomorphism 
$s_{i}: U\left(V_{i}\right)(\mathbb{A}) \rightarrow \widetilde{\mathrm{Sp}}(W)(\mathbb{A})$ such that we have the commutative diagram

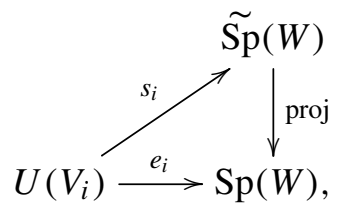

where $e_{i}$ is the embedding of $\mathrm{U}\left(V_{i}\right)$ into $\operatorname{Sp}(W)$, which is the restriction of e defined in (11). Further, $s_{i}\left(U\left(V_{i}\right)(F)\right) \subset \widetilde{\mathrm{Sp}}(W)(F)$.

If $\operatorname{dim}_{K} V_{2}=m$, we choose a character $\chi_{V_{2}}$ of $K^{\times}$such that $\left.\chi_{V_{2}}\right|_{F^{\times}}=\epsilon_{K / F}^{m}$, where $\epsilon_{K / F}$ is the quadratic character associated to the extension $K / F$. This choice determines a lifting $s_{1}: U\left(V_{1}\right) \rightarrow \widetilde{\mathrm{Sp}}(W)$, which can be explicitly formulated. Notice that two choices $\chi$ and $\chi^{\prime}$ of $\chi_{V_{2}}$ differ by a character $\mu$ of $K^{\times}$, namely $\chi=\mu \chi^{\prime}$ and $\left.\mu\right|_{F^{\times}}=1$. Then $\mu$ defines a character $\mu^{\prime}$ on $K^{1}$ by $\mu^{\prime}(x / \bar{x})=\mu(x)$, with $x \in K^{\times}$, and we have:

Lemma 3.3 [Harris et al. 1996]. $s_{1, \chi}=\left(\mu^{\prime} \circ\right.$ det $) \cdot s_{1, \chi^{\prime}}$

Doubling method. From the symplectic space $W=V_{1} \otimes V_{2}$, we can create a new symplectic space $\mathbb{W}$, which is essentially two copies of $W$, in this way: Take $W^{-}=V_{1}^{-} \otimes V_{2}$. As a vector space, $V_{1}^{-}$is the same as $V_{1}$, but the skew-Hermitian form defined on it is $-(\cdot, \cdot)_{1}$. To $\mathbb{W}=W \oplus W^{-}$, we have one dual reductive paier $\left(U\left(V_{1} \oplus V_{1}^{-}\right), U\left(V_{2}\right)\right)$. We have the following commutative diagram:

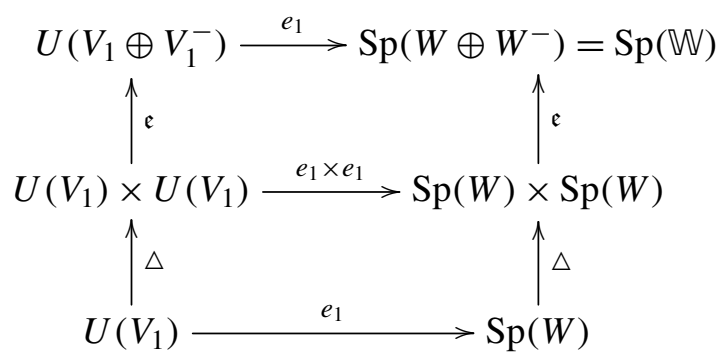

By Proposition 3.2, $\widetilde{\mathrm{Sp}}(\mathbb{W})$ splits over each of the unitary groups in the diagram above. We want to determine the compatibility among the splittings. The Weil representation of $\widetilde{\mathrm{Sp}}(\mathbb{W})$ determines an isomorphism

$$
\tilde{\mathrm{Sp}}(\mathbb{W}) \simeq \mathrm{Sp}(\mathbb{W}) \times \mathbb{C}^{\times}
$$

Group multiplications on the right hand side are described by the cocycle $c\left(g_{1}, g_{2}\right)$ :

$$
\left(g_{1}, c_{1}\right)\left(g_{2}, c_{2}\right)=\left(g_{1} g_{2}, c\left(g_{1}, g_{2}\right) c_{1} c_{2}\right)
$$


The inverse image of $\mathfrak{e}(\operatorname{Sp}(W) \times 1)$ in $\widetilde{\mathrm{Sp}}\left(W \oplus W^{-}\right)$is isomorphic to $\widetilde{\mathrm{Sp}}(W)$. We choose a lift $\tilde{\mathfrak{e}}$ of $\mathfrak{e}$ so that

$$
\tilde{\mathfrak{e}}: \widetilde{\mathrm{Sp}}(W) \times \tilde{\mathrm{Sp}}(W) \rightarrow \widetilde{\mathrm{Sp}}\left(W \oplus W^{-}\right)
$$

restricted to the $\mathbb{C}^{\times}$-component is

$$
\mathbb{C}^{\times} \times \mathbb{C}^{\times} \rightarrow \mathbb{C}^{\times}, \quad\left(c_{1}, c_{2}\right) \mapsto c_{1} \bar{c}_{2} .
$$

Since $\left(U\left(V_{1} \oplus V_{1}^{-}\right), U\left(V_{2}\right)\right)$ is a dual reductive pair of $\operatorname{Sp}(\mathbb{W})$, and $\operatorname{dim}_{K} V_{2}=m$, choose $\chi$ such that $\left.\chi\right|_{A^{x}}=\epsilon_{K / F}^{m}$. We can obtain an explicit homomorphism

$$
s_{\chi}: U\left(V_{1} \oplus V_{1}^{-}\right) \rightarrow \tilde{\mathrm{Sp}}(\mathbb{W}),
$$

so that

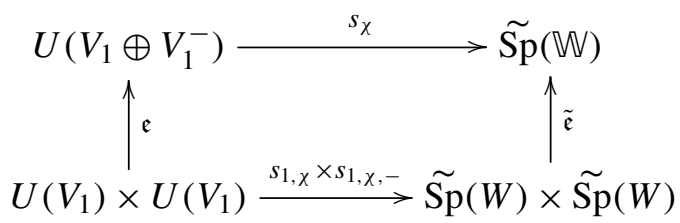

Lemma 3.4 [Harris et al. 1996]. In the commutative diagram above,

$$
\left.s_{\chi}\right|_{U\left(V_{1}\right) \times 1}=s_{1, \chi} \text { and } s_{1, \chi,-}=\left(\chi^{-1} \text { odet }\right) \cdot s_{1, \chi} .
$$

Weil representations on dual reductive pairs. Let $W=X \oplus Y$ be the complete polarization. Then $\mathbb{W}$ naturally has the polarization $\mathbb{W}=(X \oplus X) \oplus(Y \oplus Y)$. Now take the Weil representation $\omega_{\psi}$ of $\tilde{\mathrm{Sp}}(\mathbb{W})$ constructed from the standard Schrödinger model associated to this polarization. Fix a pair of characters $\chi_{n}$ and $\chi_{m}$ of $K^{\times}$with $\left.\chi_{n}\right|_{A^{\times}}=\epsilon_{K / F}^{n}$ and $\left.\chi_{m}\right|_{A^{\times}}=\epsilon_{K / F}^{m}$, where $n=\operatorname{dim}_{K} V_{1}$ and $m=\operatorname{dim}_{K} V_{2}$. These characters determine the splitting homomorphisms

$$
\begin{aligned}
s_{\chi_{m}} & : U\left(V_{1} \oplus V_{1}^{-}\right) \rightarrow \widetilde{\mathrm{Sp}}(\mathbb{W}), \\
s_{1, \chi_{m}} & : U\left(V_{1}\right) \rightarrow \widetilde{\mathrm{Sp}}(W), \\
s_{2, \chi_{m}} & : U\left(V_{2}\right) \rightarrow \widetilde{\mathrm{Sp}}(W) .
\end{aligned}
$$

Define the representation of $\mathrm{U}\left(V_{1} \oplus V_{1}^{-}\right)$to be $\omega_{\chi_{m}}=\omega_{\psi} \circ s_{\chi_{m}}$; the representations $\omega_{1, \chi_{m}}$ and $\omega_{2, \chi_{n}}$ of $\mathrm{U}\left(V_{1}\right)$ and $\mathrm{U}\left(V_{2}\right)$ can be defined similarly.

There is another polarization of $\mathbb{W}$ that we are also interested in. The skewHermitian space $V_{1} \oplus V_{1}^{-}$has the decomposition $V_{1} \oplus V_{1}^{-}=V_{d} \oplus V^{d}$ where $V_{d}=\left\{(x, x) \mid x \in V_{1}\right\}$ and $V^{d}=\left\{(x,-x) \mid x \in V_{1}\right\}$. They are maximal isotropic subspaces. Thus $\mathbb{W}=V_{d} \otimes V_{2} \oplus V^{d} \otimes V_{2}=\mathbb{W}_{d} \oplus \mathbb{W}^{d}$ is a complete polarization. We abuse the notation and still use $\omega_{\psi}$ to denote the Weil representation defined from this polarization, since one can easily distinguish representations attached to two polarizations from the context. First, let us write down the Weil representation 
$\omega_{\chi_{m}}$ of $\mathrm{U}\left(V_{1} \oplus V_{1}^{-}\right)$on $S\left(\mathbb{W}_{d}\right)$, which we are going to use in later calculations. By the Witt decomposition $V_{1} \oplus V_{1}^{-}=V_{d} \oplus V^{d}, \quad\left({ }^{A}{ }_{\left(A^{*}\right)^{-1}}\right) \in U\left(V_{1} \oplus V_{1}^{-}\right)$for $A \in \mathrm{GL}\left(V_{d}\right)$, and $\left(\begin{array}{cc}1 & B \\ & 1\end{array}\right) \in U\left(V_{1} \oplus V_{1}^{-}\right)$if $B=B^{*}$. Given $\phi(x) \in S\left(V_{d} \otimes V_{2}\right)$, we have

$$
\begin{aligned}
& \omega_{\chi_{m}}\left(\begin{array}{ll}
A & \\
& \left(A^{*}\right)^{-1}
\end{array}\right) \phi(x)=\chi_{m}(\operatorname{det} A)|\operatorname{det} A|_{K}^{m / 2} \phi\left(A^{t} x\right), \\
& \omega_{\chi_{m}}\left(\begin{array}{cc}
1 & B \\
& 1
\end{array}\right) \phi(x)=\psi\left(x^{*} B x\right) \phi(x), \\
& \omega_{\chi_{m}}\left(\begin{array}{c}
1 \\
-1
\end{array}\right) \phi(x)=\hat{\phi}(x) \text {. }
\end{aligned}
$$

Proposition 3.5. (1) Under the homomorphism $\tilde{\mathfrak{e}}: \tilde{\mathrm{Sp}}(W) \times \widetilde{\mathrm{Sp}}(W) \rightarrow \widetilde{\mathrm{Sp}}(\mathbb{W})$, we have $\omega_{\psi} \circ \tilde{\mathfrak{e}}=\omega_{\psi} \otimes \check{\omega}_{\psi}$, where $\check{\omega}_{\psi}$ is the contragredient of $\omega_{\psi}$.

(2) As the representation of $\mathrm{U}\left(V_{1}\right) \times U\left(V_{1}\right)$, we have $\omega_{\chi_{m}} \circ \mathfrak{e} \simeq \omega_{1, \chi_{m}} \otimes\left(\chi_{m} \circ \check{\omega}_{1, \chi_{m}}\right)$.

(3) The representation $\omega_{\chi_{m}} \circ \mathfrak{e} \circ \Delta$ of $\mathrm{U}\left(V_{1}\right)$ is isomorphic to the twist by $\chi_{m}$ of the linear action of $\mathrm{U}\left(V_{1}\right)$ on $S\left(\mathbb{W}_{d}\right)$, that is, for $\phi \in S\left(\mathbb{W}_{d}\right)$ and $x \in \mathbb{W}_{d}$,

$$
\omega_{\chi_{m}}(\mathfrak{e}(g, g)) \phi(x)=\chi_{m}(\operatorname{det} g) \cdot \phi(x g) .
$$

Using two polarizations of $\mathbb{W}$, two Weil representations of $\mathrm{U}\left(V_{1} \oplus V_{1}^{-}\right)$are defined above. An isometry between the two representation spaces $S((X \oplus X)(\mathbb{A}))$ and $S\left(\mathbb{W}_{d}(\mathbb{A})\right)$ can be given so that it intertwines the two representations on the spaces. Let

$$
\delta_{\psi}: S((X \oplus X)(\mathbb{A})) \rightarrow S\left(\mathbb{W}_{d}(\mathbb{A})\right)
$$

be the intertwining isometry. Identify $\mathbb{W}_{d}$ with $W$ via the map $(w,-w) \rightarrow w$ and write $w \in W$ as $w=(x, y)$ with respect to the polarization $W=X \oplus Y$. Then for $\phi \in S((X \oplus X)(\mathbb{A}))$, one has

$$
\delta_{\psi}(\phi)(w)=\int_{X(\mathbb{A})} \psi(2\langle u, y\rangle) \phi(u+x, u-x) d u,
$$

where $\langle\cdot, \cdot\rangle$ is the alternating pair on $W$. The map $\delta_{\psi}$ intertwines the action of $\widetilde{\mathrm{Sp}}(\mathbb{W})$ on those two spaces. In particular, if $\phi=\phi_{1} \otimes \bar{\phi}_{2}$ for $\phi_{1}, \phi_{2} \in S(X(\mathbb{A}))$,

$$
\delta_{\psi}\left(\phi_{1} \otimes \bar{\phi}_{2}\right)(0)=\left(\phi_{1}, \phi_{2}\right)
$$

where $(\cdot, \cdot)$ is the Hermitian inner product in the Hilbert space $L^{2}(X(\mathbb{A}))$, and

$$
\omega_{\chi_{m}}\left(i\left(g_{1}, g_{2}\right)\right) \delta_{\psi}\left(\phi_{1} \otimes \bar{\phi}_{2}\right)=\delta_{\psi}\left(\omega_{1, \chi_{m}}\left(g_{1}\right) \phi_{1} \otimes \chi_{m}\left(\operatorname{det} g_{2}\right) \check{\omega}_{1, \chi_{m}}\left(g_{2}\right) \bar{\phi}_{2}\right) .
$$


3C. Theta functions and applications. In this section, I will recall some facts about theta functions that are used in calculations of Fourier-Jacobi coefficients of Eisenstein series. More discussions about arithmetic properties of theta functions will be left to Appendix B.

Introduction. Recall $W$ has a complete polarization $W=X \oplus Y$. On $S(X(\mathbb{A}))$, there is a distribution $\theta$ defined by

$$
\theta(\phi)=\sum_{l \in X(F)} \phi(l)
$$

It can be proved that for each $\phi \in S(X(\mathbb{A}))$, the sum on the right converges absolutely. Let the Jacobi group $J(W)$ be the semidirect product of $H(W)$ and $\operatorname{Sp}(W)$. Put $\widetilde{J}(W)(\mathbb{A})=H(W)(\mathbb{A}) \cdot \widetilde{\mathrm{Sp}}(W)(\mathbb{A})$. Use $\tilde{g} \cdot \phi$ to represent the Weil representation of $\tilde{g} \in \tilde{\operatorname{Sp}}(W)(\mathbb{A})$ on $\phi$. So for each $\phi \in S(X(\mathbb{A}))$, the theta function $\theta_{\phi}$ on $\widetilde{J}(W)(\mathbb{A})$ is defined as

$$
\theta_{\phi}((w, t) \tilde{g})=\sum_{l \in X(F)} \rho_{\psi}(w, t)(\tilde{g} \cdot \phi)(l) .
$$

It is $J(W)(F)$-invariant.

When $\chi_{m}$ is chosen, the splitting $s_{1, \chi_{m}}: U\left(V_{1}\right) \rightarrow \widetilde{\mathrm{sp}}(W)$ is fixed as stated in Proposition 3.2. Let

$$
\omega\left(g_{1}, g_{2}\right) \phi(x)=\omega_{1, \chi_{m}}\left(g_{1}\right) \phi\left(g_{2}^{-1} x\right)
$$

for $g_{1} \in U\left(V_{1}\right)$ and $g_{2} \in U\left(V_{2}\right)$. Thus $\theta_{\phi}$ can also be regarded as a function on the dual reductive pair $\left(U\left(V_{1}\right), U\left(V_{2}\right)\right)$, and

$$
\theta_{\phi}\left(g_{1}, g_{2}\right)=\theta\left(\omega\left(g_{1}, g_{2}\right) \phi\right) \text {. }
$$

Theta liftings. From now on, assume that $\operatorname{dim}_{K} V_{1}=2, \operatorname{dim}_{K} V_{2}=1$ and assume $\left(V_{1},(\cdot, \cdot)_{1}\right)$ is an anisotropic skew-Hermitian space. This is exactly the situation that one will see in next sections.

Let $\Pi$ be a cuspidal representation contained in $L^{2}\left(U\left(V_{1}\right)(F) \backslash U\left(V_{1}\right)(\mathbb{A})\right)$. For convenience, given a reductive group $G$ over $F$, we use $[G]$ to denote the quotient $G(F) \backslash G(\mathbb{A})$. For each smooth $\beta \in V_{\Pi}$, where $V_{\Pi}$ is the representation space of $\Pi$, the function

$$
\theta_{\phi}^{\beta}\left(g_{2}\right)=\int_{\left[U\left(V_{1}\right)\right]} \theta_{\phi}\left(g_{1}, g_{2}\right) \beta\left(g_{1}\right) d g_{1}
$$

is well-defined, where $\theta_{\phi}\left(g_{1}, g_{2}\right)$ is given in (15). Actually, $\theta_{\phi}^{\beta}$ determines a slowly increasing function on $\left[U\left(V_{2}\right)\right]$. It is expected that $\theta_{\phi}^{\beta}\left(g_{2}\right)$ is nonzero and generates an irreducible automorphic representation on $\mathrm{U}\left(V_{2}\right)$. So $\theta_{\phi}^{\beta}$ defines a theta lifting from $\mathrm{U}\left(V_{1}\right)$ to $\mathrm{U}\left(V_{2}\right)$. 
By $(11), e\left(g_{1}, g_{2}\right) \cdot\left(v_{1} \otimes v_{2}\right)=v_{1} g_{1} \otimes g_{2}^{-1} v_{2}$. So

$$
\theta_{\phi}^{\beta}\left(g_{2}\right)=\chi_{\Pi}\left(g_{2}\right) \int_{\left[U\left(V_{1}\right)\right]} \theta_{\phi}\left(g_{1}, 1\right) \beta\left(g_{1}\right) d g_{1},
$$

where $\chi_{\Pi}$ is the central character of $\Pi$. Whether $\theta_{\phi}^{\beta}$ is zero or not only depends on the scalar $\int_{\left[U\left(V_{1}\right)\right]} \theta_{\phi}\left(g_{1}, 1\right) \beta\left(g_{1}\right) d g_{1}$. In fact, $\left|\int_{\left[U\left(V_{1}\right)\right]} \theta_{\phi}\left(g_{1}, 1\right) \beta\left(g_{1}\right) d g_{1}\right|^{2}$ can be transformed into the Rallis inner product using the Siegel-Weil formula. I am going to introduce the Siegel-Weil formula in the following and show its application in the calculation of $\left|\int_{\left[U\left(V_{1}\right)\right]} \theta_{\phi}\left(g_{1}, 1\right) \beta\left(g_{1}\right) d g_{1}\right|^{2}$.

The Siegel-Weil formula. The Siegel-Weil formula on unitary groups relates the value of a Siegel-Eisenstein series to the integral of a theta function. We temporarily return to the general case. Consider the reductive pair $(U(n, n), U(V))$ with $\operatorname{dim}_{K} V=m$. Use $S\left(V^{n}(\mathbb{A})\right)$ to denote the space of the Weil representation $\omega_{\psi}$. Here we take $S\left(V_{\infty}^{n}\right)$ to be the subspace of $L^{2}\left(V_{\infty}^{n}\right)$ called the Fock space, that is,

$$
\begin{aligned}
S\left(V_{\infty}^{n}\right)=\left\{\phi: V_{\infty}^{n} \rightarrow \mathbb{C} \mid \phi\left(v_{1}, \ldots, v_{n}\right) \psi\left(i \sum_{i=1}^{n}\left|v_{i}\right|^{2}\right)\right. & \text { is antiholomorphic as } \\
& \text { a function of } \left.v_{i} \text { for } v_{i} \in V_{\infty}\right\} .
\end{aligned}
$$

Fix $\chi_{m}$ and $\chi_{2 n}$ such that the Weil representation on the reductive pair can be defined. For $\phi \in S\left(V^{n}(\mathbb{A})\right), g \in U(n, n)(\mathbb{A})$ and $u \in U(V)(\mathbb{A})$, define such a theta integral:

$$
I(g, \phi)=\int_{[U(V)]} \theta_{\phi}(g, u) d u .
$$

On the other hand, let $\mathscr{P}^{\prime}$ be the Siegel parabolic subgroup of $\mathrm{U}(n, n)$ and $\mathscr{K}^{\prime}$ be the maximal open compact subgroup. So $\mathrm{U}(n, n)(\mathbb{A})=\mathscr{P}^{\prime}(\mathbb{A}) \mathscr{K}^{\prime}(\mathbb{A})$. For $g=p k$ with $p=\left(\begin{array}{cc}A & * \\ \left(A^{*}\right)^{-1}\end{array}\right) \in \mathscr{P}^{\prime}(\mathbb{A})$ and $k \in \mathscr{K}^{\prime}(\mathbb{A})$, put $|a(g)|_{K}=|\operatorname{det} A|_{K}$. Given $\phi \in S\left(V^{n}(\mathbb{A})\right)$, let

$$
f_{\phi, s}(g)=|a(g)|_{K}^{s-s_{0}} \omega(g, 1) \phi(0),
$$

where $s_{0}=(m-n) / 2$. Then $f_{\phi, s} \in I_{\mathscr{P}^{\prime}}^{U(n, n)}\left(\chi_{m}, s\right)$. The Siegel-Eisenstein series can be defined as

$$
E\left(g, f_{\phi, s}\right)=\sum_{\gamma \in \mathscr{P}^{\prime}(F) \backslash U(n, n)(F)} f_{\phi, s}(\gamma g) .
$$

It has been proved in many cases that $E\left(g, f_{\phi, s_{0}}\right)=I(g, \phi)$ if $E\left(g, f_{\phi, s}\right)$ is holomorphic at $s=s_{0}$ and $I(g, \phi)$ is absolutely convergent. The case we are interested in is when $n=2$ and $m=1$. By a result of Weil [1965], $I(g, \phi)$ is absolutely convergent if $V$ is anisotropic. So it is automatically satisfied if $\operatorname{dim}_{K} V=1$. 
Theorem 3.6. When $n=2$ and $m=1, E\left(g, f_{\phi, s}\right)$ is holomorphic at $s=-\frac{1}{2}$ for all $\phi \in S\left(V^{n}(\mathbb{A})\right)$, and

$$
\left.E\left(g, f_{\phi, s}\right)\right|_{s=-\frac{1}{2}}=2 I(g, \phi) .
$$

For the reductive pair $\left(\mathrm{Sp}_{n}, \mathrm{O}(V)\right)$ when $V$ is an anisotropic $F$-vector space, the Siegel-Weil formula has been proved by Kudla and Rallis [1988]. The proof of Theorem 3.6 is very similar to theirs, and is found in Appendix A. Let us return to the theta lifting question. With the Siegel-Weil formula, we are ready to calculate $\left|\int_{\left[U\left(V_{1}\right)\right]} \theta_{\phi}(g, 1) \beta(g) d g\right|^{2}$. According to (16), we have

$$
\left|\int_{\left[U\left(V_{1}\right)\right]} \theta_{\phi}(g, 1) \beta(g) d g\right|^{2}=\left(\theta_{\phi}^{\beta}, \theta_{\phi}^{\beta}\right),
$$

where $\left(\theta_{\phi}^{\beta}, \theta_{\phi}^{\beta}\right)=\int_{[U(1)]} \theta_{\phi}^{\beta}(u) \overline{\theta_{\phi}^{\beta}}(u) d u$.

Proposition 3.7. We have

$$
\left(\theta_{\phi}^{\beta}, \theta_{\phi}^{\beta}\right)=\left.\frac{1}{2} \int_{U\left(V_{1}\right)(\mathbb{A})} f_{\delta_{\psi}(\phi \otimes \bar{\phi}), s}(\mathfrak{e}(g, 1))\langle\Pi(g) \beta, \beta\rangle d g\right|_{s=-\frac{1}{2}},
$$

where $\langle\Pi(g) \beta, \beta\rangle=\int_{\left[U\left(V_{1}\right)\right]} \beta\left(g^{\prime} g\right) \overline{\beta\left(g^{\prime}\right)} d g^{\prime}$ is the matrix coefficient of the representation $\Pi$.

Proof. First,

$$
\begin{aligned}
\left(\theta_{\phi}^{\beta}, \theta_{\phi}^{\beta}\right) & =\int_{[U(1)]} \int_{\left[U\left(V_{1}\right)\right]} \theta_{\phi}(g, u) \beta(g) \int_{\left[U\left(V_{1}\right)\right]} \overline{\theta_{\phi}\left(g^{\prime}, u\right) \beta\left(g^{\prime}\right)} d g d g^{\prime} d u \\
& =\int_{\left[U\left(V_{1}\right) \times U\left(V_{1}\right)\right]} \beta(g) \overline{\beta\left(g^{\prime}\right)}\left(\int_{[U(1)]} \theta_{\phi}(g, u) \overline{\theta_{\phi}\left(g^{\prime}, u\right)} d u\right) d g d g^{\prime} .
\end{aligned}
$$

Notice that

$$
\theta_{\phi}(g, u) \overline{\theta_{\phi}\left(g^{\prime}, u\right)}=\chi_{1}^{-1}\left(\operatorname{det} g^{\prime}\right) \theta_{\delta_{\psi}(\phi \otimes \bar{\phi})}\left(\mathfrak{e}\left(g, g^{\prime}\right), u\right)
$$

where $\delta_{\psi}$ is defined by (13), and $\mathfrak{e}\left(g, g^{\prime}\right) \in U\left(V_{1} \oplus V_{1}^{-}\right)(\mathbb{A}) \simeq U(2,2)(\mathbb{A})$. So $\theta_{\delta_{\psi}(\phi \times \bar{\phi})}$ is a theta function of the reductive pair $(U(2,2), U(1))$. Applying Theorem 3.6, we have

$$
\begin{aligned}
\int_{[U(1)]} \theta_{\phi}(g, u) \overline{\theta_{\phi}\left(g^{\prime}, u\right)} d u & =\chi_{1}^{-1}\left(\operatorname{det} g^{\prime}\right) \int_{[U(1)]} \theta_{\delta_{\psi}(\phi \otimes \bar{\phi})}\left(\mathfrak{e}\left(g, g^{\prime}\right), u\right) d u \\
& =\left.\frac{1}{2} \chi_{1}^{-1}\left(\operatorname{det} g^{\prime}\right) E\left(\mathfrak{e}\left(g, g^{\prime}\right), f_{\delta_{\psi}(\phi \otimes \bar{\phi}), s}\right)\right|_{s=-\frac{1}{2}}
\end{aligned}
$$


Unfolding the Eisenstein series, we have

$$
\begin{aligned}
\int_{\left[U\left(V_{1}\right) \times U\left(V_{1}\right)\right]} \beta(g) \overline{\beta\left(g^{\prime}\right)} \chi_{1}^{-1}\left(\operatorname{det} g^{\prime}\right) E\left(\mathfrak{e}\left(g, g^{\prime}\right), f_{\delta_{\psi}(\phi \times \bar{\phi}), s}\right) d g d g^{\prime} \\
=\int_{U\left(V_{1}(\mathbb{A})\right)} f_{\delta_{\psi}(\phi \times \bar{\phi}), s}(i(g, 1))\langle\Pi(g) \beta, \beta\rangle d g .
\end{aligned}
$$

\section{Fourier-Jacobi coefficients of Eisenstein series}

In Sections $2 \mathrm{~A}$ and $2 \mathrm{~B}$, we first define the unitary group $\mathrm{U}\left(I_{\zeta}\right)$ for

$$
I_{\zeta}=\left(\begin{array}{ccc}
0 & 0 & 1 \\
0 & \zeta & 0 \\
-1 & 0 & 0
\end{array}\right)
$$

with $\zeta \in \mathrm{GL}_{2}(K)$ such that $\zeta=-\zeta^{*}$ and $\operatorname{det} \zeta \notin N_{K / F}\left(K^{\times}\right)$, and then define an Eisenstein series $E(g, f, s)$ on $\mathrm{U}\left(I_{\zeta}\right)$. In this section, we are going to use the pullback formula introduced in Section $2 \mathrm{C}$ (refer to Theorem 2.6) to calculate the Fourier-Jacobi coefficients of $E(g, f, s)$.

4A. Fourier-Jacobi coefficients of Siegel-Eisenstein series on $\mathrm{U}(3,3)$. Recall that

$$
\mathrm{U}(3,3)=\left\{g \in \mathrm{GL}_{6}(K) \mid g\left(\begin{array}{cc}
I_{3} \\
-I_{3}
\end{array}\right) g^{*}=\left(\begin{array}{c}
I_{3} \\
-I_{3}
\end{array}\right)\right\} .
$$

In Section 2B, we define a Siegel-Eisenstein series as follows: Take $\mathbb{f} \in I_{\mathscr{P}}^{U(3,3)}(\eta, s)$ and define

$$
\mathbb{E}(g, \mathbb{f}, s)=\sum_{\gamma \in \mathscr{P}(F) \backslash U(3,3)(F)} \mathbb{E}_{s}(\gamma g) .
$$

In this section, we will define the Fourier-Jacobi coefficients of this Eisenstein series, and show that if the holomorphic section $\mathbb{E}$ is chosen properly, the FourierJacobi coefficient is a product of a theta function and a Siegel-Eisenstein series on $\mathrm{U}(2,2)$.

Let $H=\left\{(x, y, t) \mid x, y \in K^{2}, t \in F\right\} \subset U(3,3)$, where

$$
(x, y, t)=\left(\begin{array}{cccc}
1 & x & t+\frac{1}{2}\left(x y^{*}-y x^{*}\right) & y \\
I_{2} & y^{*} & 0_{2} \\
& 1 & \\
& -x^{*} & I_{2}
\end{array}\right) .
$$

Notice that

$\left(x_{1}, y_{1}, t_{1}\right) \cdot\left(x_{2}, y_{2}, t_{2}\right)=\left(x_{1}+x_{2}, y_{1}+y_{2}, t_{1}+t_{2}+\frac{1}{2}\left(x_{1} y_{2}^{*}+y_{2} x_{1}^{*}\right)-\frac{1}{2}\left(x_{2} y_{1}^{*}-y_{1} x_{2}^{*}\right)\right)$. 
So if we take $\mathbb{W}_{d}=\{(x, 0,0)\}$ and $\mathbb{W}^{d}=\{(0, y, 0)\}$, then $\mathbb{W}=\mathbb{W}_{d} \oplus \mathbb{W}^{d}$ is a symplectic space with the alternating pair

$$
\left\langle\left(x_{1}, y_{1}\right),\left(x_{2}, y_{2}\right)\right\rangle=x_{1} y_{2}^{*}+y_{2} x_{1}^{*}-x_{2} y_{1}^{*}-y_{1} x_{2}^{*},
$$

and $\mathbb{W}_{d}$ and $\mathbb{W}^{d}$ are two isotropic subspaces. By the definition of Heisenberg groups in Section $3 \mathrm{~A}, H$ is a Heisenberg group associated to $\mathbb{W}$, and

$$
T=\{(0,0, t), t \in F\}
$$

is the center of $H$. There is another subgroup of $\mathrm{U}(3,3)$ :

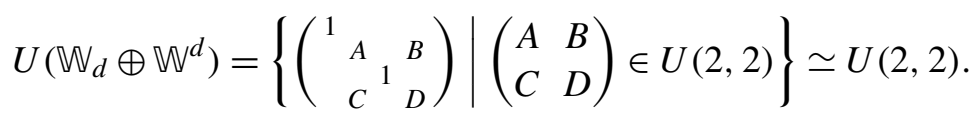

The action of $\mathrm{U}\left(W_{d} \oplus \mathbb{W}^{d}\right)$ on $H$ is

$$
\left(\begin{array}{ll}
A & B \\
C & D
\end{array}\right) \cdot(x, y, t)=\left(\begin{array}{ll}
A & B \\
C & D
\end{array}\right)^{-1}(x, y, t)\left(\begin{array}{ll}
A & B \\
C & D
\end{array}\right)=(x A+y C, x B+y D, t) .
$$

Denote by $\psi_{\mathbb{Q}}$ the additive character of $\mathbb{Q} \backslash \mathbb{A}_{\mathbb{Q}}$ with

$$
\psi_{\mathbb{Q}}\left(x_{\infty}\right)=\exp \left(2 \pi \sqrt{-1} x_{\infty}\right) \text { for } x_{\infty} \in \mathbb{R} .
$$

For $m \in F$, define an additive character $\psi_{m}$ (later in this paper, we may simply denote it by $\psi$ ) of $F \backslash \mathbb{A}$ by

$$
\psi_{m}(x)=\psi_{\mathbb{A}}\left(\operatorname{Tr}_{F / \mathbb{Q}}(m x)\right) \text { for } x \in \mathbb{A} .
$$

Define the Fourier-Jacobi coefficient of the Eisenstein series $\mathbb{E}(\cdot, \mathbb{F}, s)$ associated to $\psi$ as follows:

$$
\mathbb{E}_{\psi}(g, \mathbb{f}, s)=\int_{[T]} \mathbb{E}(t g, \mathbb{F}, s) \psi(-t) d t \quad \text { for } g \in U(3,3)(\mathbb{A}) .
$$

So $\mathbb{E}$ admits the Fourier-Jacobi expansion

$$
\mathbb{E}(g, \mathbb{E}, s)=\sum_{m \in F} \mathbb{E}_{\psi_{m}}(g, \mathbb{E}, s) .
$$

We can regard $\mathbb{E}_{\psi}$ as a function on the semidirect product of $H$ and $\mathrm{U}(2,2)$, denoted by $J_{H}$. Thus $\mathbb{E}_{\psi}(h g, \mathbb{f}, s) \in C_{\psi}^{\infty}\left(\left[J_{H}\right]\right)$, where the subindex $\psi$ means that the left action of $T$ on the functions is given by $\psi$.

Lemma 4.1. (1) Let

$$
\xi_{0}=\left(\begin{array}{ccc}
0 & 1 & \\
& I_{2} & \\
-1 & 0 & \\
& & I_{2}
\end{array}\right) \text {. }
$$

Then $\mathrm{U}(3,3)=\mathscr{P} J_{H} \sqcup \mathscr{P} \xi_{0} J_{H}$, where $\mathscr{P}$ is the Siegel parabolic subgroup of $\mathrm{U}(3,3)$. 
(2) $\mathscr{P} \backslash \mathscr{P} \xi_{0} J_{H}=\xi_{0} \cdot \mathbb{W}^{d} \backslash H \cdot \mathscr{P}^{\prime} \backslash U(2,2)=w_{3} \cdot \mathbb{W}_{d} \backslash H \cdot w_{2} \cdot \mathscr{P}^{\prime} \backslash U(2,2)$, where $\mathscr{P}^{\prime}$ is the Siegel parabolic subgroup of $\mathrm{U}(2,2)$ and $w_{n}=\left({ }_{-I_{n}} I_{n}\right) \in U(n, n)$.

Applying this lemma to $\mathbb{E}_{\psi}(h g, \mathbb{f}, s)$, we have

$$
\begin{aligned}
\mathbb{E}_{\psi}(h g, \mathbb{E}, s) & =\int_{[T]} \psi(-t) \sum_{\gamma \in \mathscr{P}(F) \backslash U(3,3)(F)} \mathbb{\mathbb { T }}_{s}(\gamma t h g) d t \\
& =\int_{[T]} \psi(-t)\left(\sum_{\gamma \in \mathscr{P}(F) \backslash \mathscr{P}(F) J_{H}(F)}+\sum_{\gamma \in \mathscr{P} \backslash \mathscr{P}(F) \xi_{0} J_{H}(F)} \mathbb{E}_{s}(\gamma t h g)\right) d t \\
& =\int_{[T]} \psi(-t) \sum_{\gamma \in w_{3} \cdot \mathbb{W}_{d}(F) \backslash H(F) \cdot w_{2} \cdot \mathscr{P}^{\prime}(F) \backslash U(2,2)(F)} \mathbb{\mathbb { t }}_{S}(\gamma t h g) d t,
\end{aligned}
$$

because $\int_{[T]} \psi(-t) \sum_{\gamma \in \mathscr{P}(F) \backslash \mathscr{P}(F) J_{H}(F)} \mathbb{E}_{S}(\gamma t h g) d t=0$.

Pick a character $\chi$ of $K^{\times}$such that $\left.\chi\right|_{A^{\times}}=\epsilon_{K / F}$ and $\chi_{\infty}(z)=|z| / z$ for $z \in K_{\infty}^{\times}$. We defined a Weil representation $\omega_{\chi}$ of $\mathrm{U}(2,2)$ on $S\left(\mathbb{W}_{d}\right)$ by $(12)$. Here what we actually use is a bit different from the conventional one. The difference is that instead of taking $\omega_{\chi}\left(w_{2}\right) \phi(x)=\hat{\phi}(x)$ we take

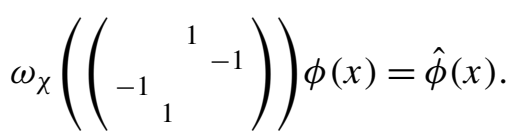

Fix such an $\omega_{\chi}$; we are going to prove that if $\mathbb{t}_{s}$ is chosen properly, then

$$
\int_{T(\mathbb{A})} \mathbb{G}_{s}\left(w_{3} t(0, y, 0) w_{2} g^{\prime}\right) \psi(-t) d t=c_{\mathbb{E}, \psi} \omega_{\chi}\left(g^{\prime}\right) \phi_{\mathbb{E}}(y) R_{s}\left(g^{\prime}\right),
$$

where $R_{S}\left(g^{\prime}\right) \in I_{\mathscr{P}^{\prime}}^{U(2,2)}\left(\eta \chi^{-1}, s\right), \quad c_{\mathbb{Q}, \psi}$ is a nonzero constant and $\phi_{\mathbb{氏}} \in S\left(\mathbb{W}_{d}(\mathbb{A})\right)$. Since the integral (23) can be decomposed into the product of local integrals, we can do the calculations place by place. The results are stated in Theorem 4.9 at the end of this section. Take a subgroup $\mathscr{K}_{(\mathbb{A})}=\prod \mathcal{K}_{v}$ of $\mathrm{U}(3,3)(\mathbb{A})$, such that $\mathscr{K}_{\infty}$ is a maximal compact subgroup of $\mathrm{U}(3,3)_{\infty}$, and $\mathscr{K}_{v}$ is a maximal open compact subgroup of $\mathrm{U}(3,3)_{v}$. Let $\mathbb{f}=\otimes^{\prime} \mathbb{E}_{v}$, with $\mathbb{E}_{\infty}$ being right $\mathscr{K}_{\infty}$-finite and $\mathbb{E}_{v}$ being spherical for almost all finite places $v$. By spherical, I mean that $\mathbb{F}_{v}$ is $\mathscr{K}_{v}$-invariant. In the following, the computations are purely local, so we omit the subindex $v$.

Case: $\mathbb{G}_{s}$ is spherical and $\chi$ is unramified. In this case, we make these assumptions:

Assumption 4.2. (1). Assume $\mathbb{E}$ is normalized, so $\mathbb{E}(1)=1$.

(2). Assume $\mathbb{W}_{d} \cap \mathscr{K}=\mathcal{O}_{K}^{2}$. The dual of $\mathbb{W}_{d} \cap \mathscr{K}$ with respect to $\psi\left(x y^{*}+y x^{*}\right)$ is defined as

$$
\left(\mathbb{W}_{d} \cap \mathscr{K}\right)^{\vee}=\left\{(0, y, 0) \in \mathbb{W}^{d} \mid \psi\left(x y^{*}+y x^{*}\right)=1 \text { for all }(x, 0,0) \in \mathbb{W}_{d} \cap \mathscr{K}\right\} .
$$

We assume $\left(\mathbb{W}_{d} \cap \mathscr{K}\right)^{\vee}$ is also $\mathscr{O}_{K}^{2}$ and $\left(\mathbb{W}_{d} \cap \mathscr{K}\right)^{\vee}=\mathbb{W}^{d} \cap \mathscr{K}$. 
Lemma 4.3. $\int_{T} \mathbb{}_{s}\left(w_{3}(0, y, t) w_{2}\right) \psi(-t) d t=\Phi_{\mathscr{O}_{K}^{2}}(y)$, where $\Phi_{\mathscr{O}_{K}^{2}}$ is the characteristic function supported on $\mathrm{O}_{K}^{2}$.

Proof. Take

$$
\boldsymbol{x}=\left(\begin{array}{ccc}
1 & & \\
-x^{*} & I_{2} & \\
& 1 & x \\
& & I_{2}
\end{array}\right) \in \mathscr{P}
$$

and notice that $\boldsymbol{x} w_{3}=w_{3}(x, 0,0)$. So,

$$
\boldsymbol{x} w_{3} t(0, y, 0) w_{2}=w_{3}\left(0, y, t+x y^{*}+y x^{*}\right)(x, 0,0) w_{2} .
$$

Then

$$
\begin{aligned}
\int_{T} \mathbb{E}_{S}\left(w_{3}(0, y, t) w_{2}\right) \psi(-t) & d t \\
& =\int_{T} \mathbb{E}_{s}\left(\boldsymbol{x} w_{3}(0, y, t) w_{2}\right) \psi(-t) d t \\
& =\int_{T} \mathbb{E}_{S}\left(w_{3}\left(0, y, t+x y^{*}+y x^{*}\right)(x, 0,0) w_{2}\right) \psi(-t) d t \\
& =\psi\left(x y^{*}+y x^{*}\right) \int_{T} \mathbb{E}_{s}\left(w_{3}(0, y, t)(x, 0,0) w_{2}\right) \psi(-t) d t .
\end{aligned}
$$

For $(0, y, 0) \in \mathbb{W}^{d} \cap \mathscr{K}$,

$$
\int_{T} \mathbb{E}_{S}\left(w_{3}(0, y, t) w_{2}\right) \psi(-t) d t=\int_{T} \mathbb{E}_{s}\left(w_{3} t\right) \psi(-t) d t=1 .
$$

If $(0, y, 0) \notin \mathbb{W}^{d} \cap \mathscr{K}$, there must exist $(x, 0,0) \in \mathbb{W}_{d} \cap \mathscr{K}$, such that $\psi\left(x y^{*}+y x^{*}\right) \neq 1$. So,

$$
\begin{aligned}
\int_{T} \mathbb{E}_{S}\left(w_{3}(0, y, t) w_{2}\right) \psi(-t) & d t \\
& =\int_{T} \mathbb{E}_{S}\left(\left(\begin{array}{ccc}
1 & & \\
-x^{*} & I_{2} & \\
& & 1 \\
& 1 & x \\
I_{2}
\end{array}\right) w_{3}(0, y, t) w_{2}\right) \psi(-t) d t \\
& =\psi\left(x y^{*}+y x^{*}\right) \int_{T} \mathbb{E}_{S}\left(w_{3}(0, y, t)(x, 0,0) w_{2}\right) \psi(-t) d t \\
& =\psi\left(x y^{*}+y x^{*}\right) \int_{T} \mathbb{E}_{S}\left(w_{3}(0, y, t)\right) \psi(-t) d t .
\end{aligned}
$$

Then $\int_{T} \mathbb{G}_{s}\left(w_{3}(0, y, t) w_{2}\right) \psi(-t) d t=0$, if $(0, y, 0) \notin \mathbb{W}^{d} \cap \mathscr{K}$.

We have the Iwasawa decomposition $\mathrm{U}(2,2)=\mathscr{P}^{\prime} \mathscr{K}^{\prime}$, where $\mathscr{P}^{\prime}$ is the Siegel parabolic subgroup of $\mathrm{U}(2,2), \mathscr{K}^{\prime}$ is the maximal open compact subgroup and 
$\mathscr{K ^ { \prime }}=\mathscr{K} \cap U(2,2)$. Take $g^{\prime}=p^{\prime} k^{\prime}=\left(\begin{array}{cc}a & b \\ \left(a^{*}\right)^{-1}\end{array}\right) k^{\prime} \in U(2,2)$, where $k^{\prime} \in \mathscr{K}^{\prime}$. Then

$$
\begin{aligned}
\int_{T} \mathbb{E}_{s}\left(w_{3}(0, y, t) w_{2}\right. & \left.\left(\begin{array}{cc}
a & b \\
\left(a^{*}\right)^{-1}
\end{array}\right) k^{\prime}\right) \psi(-t) d t \\
& =\int_{T} \mathbb{E}_{s}\left(w_{3}(0, y, t)\left(\begin{array}{cc}
\left(a^{*}\right)^{-1} \\
-b & a
\end{array}\right)\right) \psi(-t) d t \\
& =\int_{T} \mathbb{E}_{s}\left(\left(\begin{array}{cc}
a & b \\
& \left(a^{*}\right)^{-1}
\end{array}\right) w_{3}(-y b, y a, t)\right) \psi(-t) d t \\
& =\eta(\operatorname{det} a)|\operatorname{det} a|^{s+\frac{3}{2}} \int_{T} \mathbb{E}_{s}\left(w_{3}\left(0, y a, t+y b a^{*} y^{*}\right)\right) \psi(-t) d t \\
& =\eta(\operatorname{det} a)|\operatorname{det} a|^{s+\frac{3}{2}} \psi\left(y b a^{*} y^{*}\right) \Phi_{\mathscr{O}_{K}^{2}}(y a) \\
& =\chi(\operatorname{det} a)|\operatorname{det} a|^{\frac{1}{2}} \Phi_{\mathscr{O}_{K}^{2}}(y a) \psi\left(y b a^{*} y^{*}\right) \eta \chi^{-1}(\operatorname{det} a)|\operatorname{det} a|^{s+1} \\
& =\omega_{\chi}\left(p^{\prime}\right) \Phi_{\mathscr{O}_{K}^{2}}(y) R_{s}\left(g^{\prime}\right) .
\end{aligned}
$$

Notice that $\omega_{\chi}\left(k^{\prime}\right) \Phi_{\mathscr{O}_{K}^{2}}=\Phi_{\mathscr{O}_{K}^{2}}$. So

$$
\int_{T} \mathbb{G}_{S}\left(w_{3}(0, y, t) w_{2} g^{\prime}\right) \psi(-t) d t=\omega_{\chi}\left(g^{\prime}\right) \Phi_{\mathscr{O}_{K}^{2}}(y) R_{S}\left(g^{\prime}\right)
$$

with normalized spherical $R_{s} \in I_{\mathscr{P}^{\prime}}^{U(2,2)}\left(\eta \chi^{-1}, s\right)$.

Archimedean places. Let $\mathrm{U}(n)=\left\{u \in \mathrm{GL}_{n}(\mathbb{C}) \mid u u^{*}=I_{n}\right\}$ be the unitary group of degree $\mathrm{n}$ at an archimedean place. Take such an embedding:

$$
\begin{aligned}
\mathfrak{e}: U(3) \times U(3) & \mapsto U(3,3), \\
(u, v) & \mapsto \mathfrak{e}(u, v)=\left(\begin{array}{cc}
-\frac{i}{2} I_{3} & \frac{i}{2} I_{3} \\
\frac{1}{2} I_{3} & \frac{1}{2} I_{3}
\end{array}\right)\left(\begin{array}{cc}
u & \\
v
\end{array}\right)\left(\begin{array}{cc}
i I_{3} & I_{3} \\
-i I_{3} & I_{3}
\end{array}\right) .
\end{aligned}
$$

The Hermitian domain of a unitary group is defined in (2). Choose an initial point in the Hermitian domain of $\mathrm{U}(3,3)$ to be $i=i I_{3}$, where we fix $i=\sqrt{-1}$ once and for all. From Proposition 2.1, one choice of a maximal compact subgroup at an archimedean place for $\mathrm{U}(3,3)$ is $\mathscr{K}=\{g \in U(3,3) \mid g \boldsymbol{i}=\boldsymbol{i}\}$. Notice that the image of the embedding $\mathfrak{e}$ defined above is exactly $\mathscr{K}$. So $\mathscr{K} \stackrel{\mathfrak{e}}{\simeq} U(3) \times U(3)$. Recall that $\mathrm{U}(2,2)$, which is isomorphic to $\mathrm{U}\left(\mathbb{W}_{d} \oplus \mathbb{W}^{d}\right)$, is naturally embedded into $\mathrm{U}(3,3)$ (refer to (19)). So we can choose compatibly the maximal compact subgroup $\mathscr{K}^{\prime}$ of $\mathrm{U}(2,2)$ by $\mathscr{K}^{\prime}=\mathscr{K} \cap U(2,2)$, and obviously $\mathscr{K}^{\prime} \simeq U(2) \times U(2)$. The initial point $\boldsymbol{i}^{\prime}=i I_{2}$ of the Hermitian domain of $\mathrm{U}(2,2)$ is invariant under the action of elements in $\mathscr{K}^{\prime}$.

At archimedean places, take a weight $(0, k)$ section $\mathbb{V}_{k}$ such that

$$
\mathbb{F}_{k}(g)=j(g, \boldsymbol{i})^{-k},
$$


where $j$ is the automorphy factor defined in (3). Assume $\eta$ satisfies $\eta(z)=\bar{z}^{l^{\prime}} / z^{l}$;

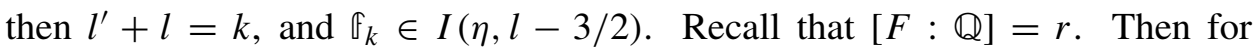
$m \in F$, let $m_{\infty}=\left(m_{1}, m_{2}, \ldots, m_{r}\right) \in F_{\infty} \simeq \mathbb{R}^{r}$. Given $z=\left(z_{1}, z_{2}, \ldots, z_{r}\right) \in F_{\infty}$, $\psi(z)=\exp \left(2 \pi i m_{1} z_{1}\right) \exp \left(2 \pi i m_{2} z_{2}\right) \cdots \exp \left(2 \pi i m_{r} z_{r}\right)$.

Proposition 4.4. Take $\mathbb{\llbracket}_{k}$ and $\psi$ as above. We have

$$
\int_{T} \psi(-t) \mathbb{f}_{k}\left(w_{3}(0, y, t) w_{2} g^{\prime}\right) d t=c_{\psi} \omega_{\chi}\left(g^{\prime}\right) \phi_{\mathbb{E}_{k}}(y) R_{k-1}\left(g^{\prime}\right),
$$

where $g^{\prime} \in U(2,2), \phi_{\mathbb{G}_{k}}(y)=\psi\left(\right.$ iyy $\left.y^{*}\right), \chi$ satisfies $\chi(z)=|z| / z$ for $z \in K_{\infty}^{\times}$, and

$$
c_{\psi}=\frac{(-2 \pi i)^{k r}\left(m_{1} m_{2} \cdots m_{r}\right)^{k-1} e^{-2 \pi\left(m_{1}+m_{2}+\cdots+m_{r}\right)}}{[(k-1) !]^{r}} .
$$

The function $R_{k-1}$ satisfies $R_{k-1}\left(g^{\prime}\right)=j\left(g^{\prime}, i^{\prime}\right)^{-k+1}$. As an induced representation on $\mathrm{U}(2,2)$, it is in $I\left(\eta \chi^{-1}, l-\frac{3}{2}\right)$ and is a weight $(0, k-1)$ section.

Proof. For simplicity, we prove this proposition under the assumption that $r=1$, from which the general result should be easily derived. So regard $m$ as a real number first.

Step 1. Let $g^{\prime}=I_{4}$. We have

$$
\begin{aligned}
\int_{\mathbb{R}} \psi(-t) \mathbb{\mathbb { F }}_{k}\left(w_{3}(0, y, t) w_{2}\right) d t & =(-1)^{k} \int_{\mathbb{R}} \psi(-t)\left(t+i+i y y^{*}\right)^{-k} d t \\
& =(-1)^{k} \psi\left(i y y^{*}+i\right) \int_{\mathbb{R}+i+i y y^{*}} \psi(-t) t^{-k} d t \\
& =\frac{(-2 \pi i)^{k} m^{k-1} e^{-2 \pi m}}{(k-1) !} \psi\left(i y y^{*}\right) .
\end{aligned}
$$

This calculation hints that we should take $\phi_{\mathbb{E}_{k}}$ to be $\psi\left(i y y^{*}\right)$.

Step 2. Replace $g^{\prime}$ by

$$
p^{\prime} g^{\prime}=\left(\begin{array}{ll}
a & \\
& \left(a^{*}\right)^{-1}
\end{array}\right)\left(\begin{array}{ll}
I_{2} & b \\
& I_{2}
\end{array}\right) g^{\prime} .
$$

If we assume that

$$
\int_{\mathbb{R}} \psi(-t) \mathbb{f}_{k}\left(w_{3}(0, y, t) w_{2} g^{\prime}\right) d t=\frac{(-2 \pi i)^{k} m^{k-1} e^{-2 \pi m}}{(k-1) !} \psi\left(i y y^{*}\right) R_{k-1}\left(g^{\prime}\right),
$$


then

$$
\begin{aligned}
& \int_{\mathbb{R}} \psi(-t) \mathbb{f}_{k}\left(w_{3}(0, y, t) w_{2} p^{\prime} g^{\prime}\right) d t \\
& =(\overline{\operatorname{det} a})^{k} \psi\left(y a b a^{*} y^{*}\right) \int_{\mathbb{R}} \psi(-t) \mathbb{\llbracket}_{k}\left(w_{3}(0, y a, t) w_{2} g^{\prime}\right) d t \\
& =\frac{(-2 \pi i)^{k} m^{k-1} e^{-2 \pi m}}{(k-1) !}(\overline{\operatorname{det} a})^{k} \psi\left(y a b a^{*} y^{*}\right) \psi\left(i y a a^{*} y^{*}\right) R_{k-1}\left(g^{\prime}\right) \\
& =\frac{(-2 \pi i)^{k} m^{k-1} e^{-2 \pi m}}{(k-1) !} \omega_{\chi}\left(p^{\prime} g^{\prime}\right) \phi_{\mathbb{V}_{k}}(y) R_{k-1}\left(p^{\prime} g^{\prime}\right) .
\end{aligned}
$$

Step 3. Take $g^{\prime}=w_{2} n^{\prime}=w_{2}\left(\begin{array}{cc}I_{2} & b \\ & I_{2}\end{array}\right)$. Note that we take

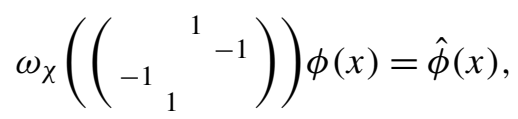

so one easily checks that at archimedean places, $\omega_{\chi}\left(w_{2}\right) \phi(x)=-\hat{\phi}(x)$. Then,

$$
\begin{aligned}
& \int_{\mathbb{R}} \psi(-t) \mathbb{\llbracket}_{k}\left(w_{3}(0, y, t) w_{2} w_{2} n^{\prime} d t\right. \\
& =(-1)^{k} \int_{\mathbb{R}} \psi(-t) \operatorname{det}\left(\begin{array}{cc}
t+i & -y \\
-y^{*} & b+i
\end{array}\right)^{-k} d t \\
& =(-1)^{k} \int_{\mathbb{R}} \psi(-t) \operatorname{det}(b+i)^{-k}\left(t+i-y(b+i)^{-1} y^{*}\right)^{-k} d t \\
& =\frac{(-2 \pi i)^{k} m^{k-1} e^{-2 \pi m}}{(k-1) !} \operatorname{det}(b+i)^{-k} \psi\left(-y(b+i)^{-1} y^{*}\right) \\
& =\frac{(-2 \pi i)^{k} m^{k-1} e^{-2 \pi m}}{(k-1) !} \omega_{\chi}\left(w_{2} n^{\prime}\right) \phi_{\mathbb{\varpi}_{k}}(y) R_{k-1}\left(w_{2} n^{\prime}\right) .
\end{aligned}
$$

Here we use that the Fourier transform of $\psi\left(y(i+b) y^{*}\right)$ is $\operatorname{det}(1-i b)^{-1} \psi(y(-i-$ b) $\left.)^{-1} y^{*}\right)$.

Step 4. Let $g^{\prime}=w_{2} n_{1}^{\prime} w_{2} n_{2}^{\prime}$, where $n_{1}^{\prime}=\left(\begin{array}{cc}I_{2} & b_{1} \\ & I_{2}\end{array}\right)$ and $n_{2}^{\prime}=\left(\begin{array}{cc}I_{2} & b_{2} \\ & I_{2}\end{array}\right)$. Both $b_{1}$ and $b_{2}$ are $2 \times 2$ Hermitian matrices. We have a decomposition

$$
\begin{aligned}
& w_{2} n_{1}^{\prime} w_{2} n_{2}^{\prime}= w_{2}\left(\begin{array}{ll}
I_{2} & b_{1} \\
& I_{2}
\end{array}\right)\left(\begin{array}{cc}
\frac{1}{\sqrt{b_{2}^{2}+1}} & \frac{-b_{2}}{\sqrt{b_{2}^{2}+1}} \\
\sqrt{b_{2}^{2}+1}
\end{array}\right) \mathfrak{e}\left(\frac{-b_{2}+i}{\sqrt{b_{2}^{2}+1}}, \frac{-b_{2}-i}{\sqrt{b_{2}^{2}+1}}\right) \\
&=\left.\begin{array}{cc}
\sqrt{b_{2}^{2}+1} & \\
1 / \sqrt{b_{2}^{2}+1}
\end{array}\right) w_{2}\left(\begin{array}{c}
I_{2}-b_{2}+\sqrt{b_{2}^{2}+1} b_{1} \sqrt{b_{2}^{2}+1} \\
I_{2}
\end{array}\right) \\
& \cdot \mathfrak{e}\left(\frac{-b_{2}+i}{\sqrt{b_{2}^{2}+1}}, \frac{-b_{2}-i}{\sqrt{b_{2}^{2}+1}}\right) .
\end{aligned}
$$


From Steps 2 and 3, one can see that when $g^{\prime} \in \mathscr{P}^{\prime} w_{2} \mathcal{N}^{\prime} \subset U(2,2)$, where $\mathcal{N}^{\prime}$ is the unipotent radical of $\mathscr{P}^{\prime}$, the equality (24) holds. Then let us prove that when taking $g^{\prime}=w_{2} n_{1}^{\prime} w_{2} n_{2}^{\prime}$, the equality still holds. We have

$$
\mathbb{G}_{k}\left(w_{3}(0, y, t) w_{2} w_{2} n_{1} w_{2} n_{2}\right)=\mathbb{E}_{k}\left(w_{3}(0, y, t) w_{2} p^{\prime} w_{2} n^{\prime}\right) \operatorname{det}\left(\frac{b_{2}+i}{\sqrt{b_{2}^{2}+1}}\right)^{-k},
$$

where

$$
p^{\prime}=\left(\begin{array}{cc}
\sqrt{b_{2}^{2}+1} & \\
& 1 / \sqrt{b_{2}^{2}+1}
\end{array}\right) \quad \text { and } \quad n^{\prime}=\left(\begin{array}{c}
I_{2}-b_{2}+\sqrt{b_{2}^{2}+1} b_{1} \sqrt{b_{2}^{2}+1} \\
I_{2}
\end{array}\right) .
$$

So

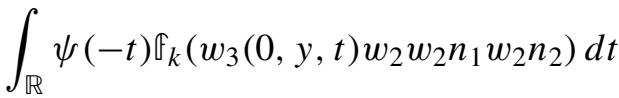

$$
\begin{aligned}
& =\operatorname{det}\left(\frac{b_{2}+i}{\sqrt{b_{2}^{2}+1}}\right)^{-k} \int_{\mathbb{R}} \psi(-t) \mathbb{F}_{k}\left(w_{3}(0, y, t) w_{2} p^{\prime} w_{2} n^{\prime}\right) d t \\
& =\frac{(-2 \pi i)^{k} m^{k-1} e^{-2 \pi m}}{(k-1) !} \operatorname{det}\left(\frac{b_{2}+i}{\sqrt{b_{2}^{2}+1}}\right)^{-k} \omega_{\chi}\left(p^{\prime} w_{2} n^{\prime}\right) \phi_{\bigoplus_{k}}(y) R_{k-1}\left(p^{\prime} w_{2} n^{\prime}\right) \\
& =\frac{(-2 \pi i)^{k} m^{k-1} e^{-2 \pi m}}{(k-1) !} \operatorname{det}\left(\frac{b_{2}+i}{\sqrt{b_{2}^{2}+1}}\right)^{-1} \omega_{\chi}\left(p^{\prime} w_{2} n^{\prime}\right) \phi_{\mathbb{V}_{k}}(y) R_{k-1}\left(w_{2} n_{1} w_{2} n_{2}\right) .
\end{aligned}
$$

Note that

$$
\omega_{\chi}\left(p^{\prime} w_{2} n^{\prime}\right) \phi_{\mathbb{F}_{k}}(y)=-\frac{1}{\operatorname{det}\left(\sqrt{b_{2}^{2}+1}\right)} \frac{1}{\operatorname{det}\left(\frac{b_{2} i+1}{b_{2}^{2}+1}-b_{1} i\right)} \psi\left(y\left(\frac{b_{2}-i}{b_{2}^{2}+1}-b_{1}\right)^{-1} y^{*}\right) .
$$

Also,

$\omega_{\chi}\left(w_{2} n_{1}^{\prime} w_{2} n_{2}^{\prime}\right) \phi_{\mathbb{\varpi}_{k}}(y)$

$$
=\frac{1}{\operatorname{det}\left(1-b_{2} i\right)} \frac{1}{\operatorname{det}\left(\left(1-b_{2} i\right)^{-1}-b_{1} i\right)} \psi\left(y\left(\left(b_{2}+i\right)^{-1}-b_{1}\right)^{-1} y^{*}\right) .
$$

By comparison,

$$
\int_{\mathbb{R}} \psi(-t) \mathbb{\mathfrak { F }}_{k}\left(w_{3}(0, y, t) w_{2} g^{\prime}\right) d t=\frac{(-2 \pi i)^{k} m^{k-1} e^{-2 \pi m}}{(k-1) !} \omega_{\chi}\left(g^{\prime}\right) \phi_{\mathbb{F}_{k}}(y) R_{k-1}\left(g^{\prime}\right)
$$

for $g^{\prime}=w_{2} n_{1}^{\prime} w_{2} n_{2}^{\prime}$.

Step 5. It is known that elements in $\mathrm{U}(2,2)$ can be generated by $w_{2}$ and $p^{\prime} \in \mathscr{P}^{\prime}$, where $\mathscr{P}^{\prime}$ is the Siegel parabolic subgroup of $\mathrm{U}(2,2)$. The following lemma shows how these elements generate $\mathrm{U}(2,2)$. 
The proof is straightforward, so let me skip it. In Steps 1 to 4, we have verified that

$$
\int_{\mathbb{R}} \psi(-t) \mathbb{f}_{k}\left(w_{3}(0, y, t) w_{2} g^{\prime}\right) d t=\frac{(-2 \pi i)^{k} m^{k-1} e^{-2 \pi m}}{(k-1) !} \omega_{\chi}\left(g^{\prime}\right) \phi_{\mathbb{G}_{k}}(y) R_{k-1}\left(g^{\prime}\right)
$$

for $g^{\prime}$ in each of the subsets of $\mathrm{U}(2,2)$.

For each archimedean place, the computations are exactly as above as long as we change $m$ to the corresponding $m_{i}$. Putting them together, we can prove the proposition.

$\mathbb{F}_{s}$ is not spherical. Denote the set of places where $\mathbb{E}_{s}$ is not the spherical element in the induced representation space by $S$. When $\mathbb{\varpi}_{s}$ is not spherical, we make such a choice: $\mathbb{E}_{s} \in I(\eta, s)$ and $\mathbb{E}_{s}$ is supported in the big cell $\mathscr{P} w_{3} \mathscr{P}$ such that $\mathbb{I}_{s}\left(w_{3} n(b)\right)=$ $\Phi(b)$, where $n(b)=\left(\begin{array}{cc}I_{3} & b \\ & I_{3}\end{array}\right)$, and $b$ is a $3 \times 3$ Hermitian matrix. Let $b=\left(\begin{array}{cc}t & y \\ y^{*} & b^{\prime}\end{array}\right)$, where $t \in F$ and $b^{\prime}$ is a $2 \times 2$ Hermitian matrix. Assume $\Phi(b)=\phi^{\prime}(t) \phi(y) \Phi^{\prime}\left(b^{\prime}\right)$ such that $\phi, \phi^{\prime}, \Phi^{\prime}$ are all Bruhat-Schwartz functions. Further we make these assumptions concerning $\mathbb{E}_{s}$ and the additive character $\psi$ :

Assumption 4.6. (1) The set $S$ includes all the places where $\eta$ or $\chi$ is ramified. In another words, when $\chi$ or $\eta$ is ramified, we should take $\mathbb{F}_{s}$ supported on the big cell, and as long as such an $\mathbb{E}_{s}$ satisfies the following two assumptions, Theorem 4.9 can be derived. However, for the sake of later computations in Remark 4.14, we need to further assume (28).

(2) For $b^{\prime} \in \operatorname{supp} \Phi^{\prime}$ and $y \in \operatorname{supp} \phi$, we have $\psi\left(y b^{\prime} y^{*}\right)=1$.

(3) When $t \in \operatorname{supp} \phi^{\prime}$, we have $\psi(t)=1$.

Remark 4.7. The assumptions above are in general quite weak. In practice, we may first determine $\Phi^{\prime}$ and $\phi$. Because they are both compactly supported, as a set of $F,\left\{y b^{\prime} y^{*} \mid y \in \operatorname{supp} \Phi^{\prime}, b^{\prime} \in \operatorname{supp} \phi\right\}$ must be compact. So there exists an additive character $\psi$ (in fact, infinitely many such characters) that is constant of value 1 on this set. Moreover, we can even choose a universal $\psi$ that is independent of the conductors of $\eta$ and $\chi$. Then based on Assumption 4.6(3), one can determine the function $\phi^{\prime}$. Then in this way, we can determine $\Phi$, and in turn $\mathbb{E}_{s}$.

Lemma 4.8. Suppose $R_{S}$ is in $I_{\mathscr{P}^{\prime}}^{U(2,2)}\left(\eta \chi^{-1}, s\right)$ and is supported on the big cell $\mathscr{P}^{\prime} w_{2} \mathscr{P}^{\prime}$, such that

$$
R_{S}\left(w_{2}\left(\begin{array}{cc}
I_{2} & b^{\prime} \\
& I_{2}
\end{array}\right)\right)=\Phi^{\prime}\left(b^{\prime}\right)
$$

Let $\phi_{\mathbb{T}_{s}}(y)=\hat{\phi}(-y)$. Then

$$
\int_{T} \psi(-t) \mathbb{E}_{s}\left(w_{3}(0, y, t) w_{2} g^{\prime}\right) d t=\left(\int_{T} \psi(-t) \phi^{\prime}(t) d t\right) \omega_{\chi}\left(g^{\prime}\right) \phi_{\mathbb{Q}_{s}}(y) R_{S}\left(g^{\prime}\right) .
$$


Proof. In order that $w_{3}(0, y, t) w_{2} g^{\prime} \in \mathscr{P} w_{3} \mathscr{P}$, it must be that $g^{\prime}$ is in $\mathscr{P}^{\prime} w_{2} \mathscr{P}^{\prime}$, which implies that $R_{s}$ is supported on $\mathscr{P}^{\prime} w_{2} \mathscr{P}^{\prime}$. Let $g^{\prime}=w_{2} n^{\prime}\left(b^{\prime}\right)=w_{2}\left(\begin{array}{cc}I_{2} & b^{\prime} \\ I_{2}\end{array}\right)$ for $n^{\prime}\left(b^{\prime}\right) \in \mathcal{N}^{\prime}$; then

$$
\begin{aligned}
\int_{T} \psi(-t) \mathbb{E}_{k}\left(w_{3}(0, y, t) w_{2} w_{2} n^{\prime}\left(b^{\prime}\right)\right) d t \\
=\int_{T} \psi(-t) \Phi\left(\left(\begin{array}{cc}
t & y \\
y^{*} & b^{\prime}
\end{array}\right)\right) d t \\
=\left(\int_{T} \psi(-t) \phi^{\prime}(t) d t\right) \phi(y) \Phi^{\prime}\left(b^{\prime}\right) \\
=\left(\int_{T} \psi(-t) \phi^{\prime}(t) d t\right) \omega_{\chi}\left(w_{2} n^{\prime}\left(b^{\prime}\right)\right) \phi_{\mathbb{T}_{s}}(y) R_{S}\left(w_{2} n^{\prime}\left(b^{\prime}\right)\right) .
\end{aligned}
$$

Let me summarize the local computations of the three cases above in the following

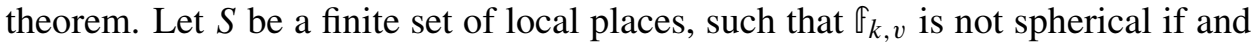
only if $v \in S$.

Theorem 4.9. For a Hecke character $\eta$ of $K$ and $\eta_{\infty}(z)=\bar{z}^{k-l} / z^{l}$, take

$$
\mathbb{E}_{k} \in I_{\mathscr{P}(\mathrm{A})}^{U(3,3)(\mathbb{A})}\left(\eta, l-\frac{3}{2}\right) .
$$

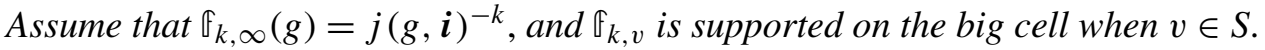
Choose a Hecke character $\chi$ of $K$ so that $\chi_{\infty}(z)=|z| / z$. Fix an additive character $\psi$ of $F$ as in (20). When $\llbracket_{k}, \chi$ and $\psi$ satisfy Assumptions 4.2 and 4.6, we have

$$
\mathbb{E}_{\psi}\left(h g^{\prime}\right)=c_{\mathbb{F}_{k}, \psi} \theta_{\Phi_{\mathbb{F}_{k}}}\left(h w_{2}^{-1} g^{\prime}\right) \mathscr{E}\left(g^{\prime}, R_{k-1}\right) \quad \text { for } h \in H(\mathbb{A}), g^{\prime} \in U(2,2)(\mathbb{A}),
$$

where

$$
c_{\varpi_{k}, \psi}=c_{\psi} \prod_{v \in S} \operatorname{vol}\left(\operatorname{supp} \phi_{v}^{\prime}\right),
$$

$c_{\psi}$ is given in Proposition 4.4, $\phi_{\mathbb{F}_{k}} \in S\left(\mathbb{W}^{d}(\mathbb{A})\right)$, and $R_{k-1} \in I_{\mathscr{P}^{\prime}}^{U(2,2)(\mathbb{A})}\left(\eta \chi^{-1}, l-\frac{3}{2}\right)$. Specifically, $R_{k-1, \infty}\left(g^{\prime}\right)=j\left(g^{\prime}, \boldsymbol{i}^{\prime}\right)^{-k+1}$ and $R_{k-1, v}$ is normalized and spherical when $v \notin S$. Otherwise, $R_{k-1, v}$ is supported on the big cell. The Siegel-Eisenstein series $\mathscr{E}$ is associated to $R_{k-1}$.

Proof. Recall (22):

$$
\mathbb{E}_{\psi}\left(h g^{\prime}, \mathbb{F}_{k}\right)=\int_{[T]} \psi\left(-t^{\prime}\right) \sum_{\substack{\gamma \in w_{3} \cdot \mathbb{W}_{d}(F) \backslash H(F) \cdot w_{2} \\ \mathscr{P}^{\prime}(F) \backslash U(2,2)(F)}} \mathbb{E}_{k}\left(\gamma t^{\prime} h g^{\prime}\right) d t^{\prime} .
$$

Let $h=(x, y, t) \in H(\mathbb{A})$; then

$$
\begin{aligned}
\left.\mathbb{\Vdash}_{k}\left(w_{3} h w_{2} g^{\prime}\right)\right) & =\mathbb{\Vdash}_{k}\left(w_{3}(x, 0,0)\left(0, y, t-\frac{1}{2}\left(x y^{*}+y x^{*}\right)\right) w_{2} g^{\prime}\right) \\
& =\mathbb{\Vdash}_{k}\left(w_{3}\left(0, y, t-\frac{1}{2}\left(x y^{*}+y x^{*}\right)\right) w_{2} g^{\prime}\right) .
\end{aligned}
$$


So we get

$$
\begin{aligned}
& \mathbb{E}_{\psi}\left(h g^{\prime}, \mathbb{f}_{k}\right) \\
& =\int_{T(\mathbb{A})} \psi\left(-t^{\prime}\right) \sum_{\substack{y_{0} \in \mathbb{W}^{d}(F) \\
g_{0} \in \mathscr{P}^{\prime}(F) \backslash U(2,2)(F)}} \mathbb{F}_{k}\left(w_{3}\left(0, y_{0}, t^{\prime}\right) w_{2} g_{0} h g^{\prime}\right) d t^{\prime} \\
& =\int_{T(\mathbb{A})} \psi\left(-t^{\prime}\right) \sum_{\substack{y_{0} \in \mathbb{W}^{d}(F) \\
g_{0} \in \mathscr{P}^{\prime}(F) \backslash U(2,2)(F)}} \mathbb{F}_{k}\left(w_{3}\left(0, y_{0}, t^{\prime}\right)\left((x, y) g_{0}^{-1} w_{2}^{-1}, t\right) w_{2} g_{0} g^{\prime}\right) d t^{\prime} \\
& =c_{\psi} \prod_{v \in S} \int_{T_{v}} \psi_{v}\left(-t^{\prime}\right) \mathbb{F}_{k, v}\left(w_{3}\left(0,0, t^{\prime}\right)\right) d t^{\prime} \\
& \sum_{\substack{y_{0} \in \mathbb{W}^{d}(F) \\
g_{0} \in \mathscr{P}^{\prime}(F) \backslash U(2,2)(F)}} \omega_{\chi}\left(\left((x, y) g_{0}^{-1} w_{2}^{-1}, t\right) g_{0} g\right) \phi_{\mathbb{F}_{k}}\left(y_{0}\right) R_{k-1}\left(g_{0} g^{\prime}\right) .
\end{aligned}
$$

Notice that $\int_{T_{v}} \psi_{v}\left(-t^{\prime}\right) \mathbb{E}_{k, v}\left(w_{3}\left(0,0, t^{\prime}\right)\right) d t^{\prime}=\operatorname{vol}\left(\operatorname{supp} \phi_{v}^{\prime}\right)$ for $v \in S$. So

$$
\begin{aligned}
& \mathbb{E}_{\psi}\left(h g^{\prime}, \mathbb{F}_{k}\right)=c_{\mathbb{G}_{k}, \psi} \theta_{{\mathbb{F}_{k}}_{k}}\left(\left((x, y) w_{2}^{-1}, t\right) g^{\prime}\right) \mathscr{E}\left(g^{\prime}, R_{k-1}\right) \\
& =c_{\mathbb{f}_{k}, \psi} \theta_{\phi_{\mathbb{F}_{k}}}\left(h w_{2}^{-1} g^{\prime}\right) \mathscr{E}\left(g^{\prime}, R_{k-1}\right) \text {. }
\end{aligned}
$$

4B. Fourier-Jacobi coefficients of Eisenstein series on $\mathrm{U}(3,1)$. In this section, I will define the Fourier-Jacobi coefficients of the Eisenstein series $E(g, f, s)$ on $\mathrm{U}\left(I_{\zeta}\right)$. If this $E(g, f, s)$ is from the pullback of a Siegel-Eisenstein series $\mathbb{E}(\cdot, \mathbb{E}, s)$, then by applying the pullback formula (Theorem 2.6 ) and results about the Fourier-Jacobi coefficients of $\mathbb{E}(\cdot, \mathbb{E}, s)$ (Theorem 4.9), we will get formulas for the Fourier-Jacobi coefficients of $E(g, f, s)$.

Definitions. Let $P$ be the only nontrivial parabolic subgroup $P$ of $\mathrm{U}\left(I_{\zeta}\right)$; then the unipotent radical of $P$ is

$$
N=\left\{\left(\begin{array}{ccc}
1 & x & t+x \zeta x^{*} / 2 \\
& I_{2} & \zeta x^{*} \\
& 1
\end{array}\right) \mid t \in F\right\}
$$

From another point of view, $N$ can be regarded as a Heisenberg group attached to a 4-dimensional symplectic space $W$ of $F$. Using conventional notation, denote

$$
\left(\begin{array}{ccc}
1 & x & t+x \zeta x^{*} / 2 \\
& I_{2} & \zeta x^{*} \\
& 1
\end{array}\right) \quad \text { by }(x, t)
$$

then

$$
\left(x_{1}, t_{1}\right)\left(x_{2}, t_{2}\right)=\left(x_{1}+x_{2}, t_{1}+t_{2}+\left\langle x_{1}, x_{2}\right\rangle / 2\right),
$$


where $\langle\cdot, \cdot\rangle$ represents the alternating pair on $W$, and

$$
\left\langle x_{1}, x_{2}\right\rangle=x_{1} \zeta x_{2}^{*}-x_{2} \zeta x_{1}^{*} .
$$

The degree two unitary group $\mathrm{U}(\zeta)=\left\{u \mid u \zeta u^{*}=\zeta\right\}$ is a subgroup of $\mathrm{U}\left(I_{\zeta}\right)$, and has an action on $N$ by $u \cdot(x, t)=u^{-1}(x, t) u=(x u, t)$.

Given the additive character $\psi$ as in (20), define the Fourier-Jacobi coefficient of $E(g, f, s)$ as

$$
E_{\psi}(n u, f, s)=\int_{[T]} \psi(-t) E(t n u, f, s) d t
$$

for $u \in U(\zeta)(\mathbb{A})$ and $n \in N(\mathbb{A})$, where $T$ is the center of $N$. So $E_{\psi} \in C_{\psi}^{\infty}([N U(\zeta)])$. Pullback of $\mathbb{E}_{\psi}$. Recall in (8), we define an embedding $\mathfrak{e}: U\left(I_{\zeta}\right) \times U(\zeta) \hookrightarrow U(3,3)$. See that

$$
\left.\mathfrak{e}\right|_{U(\zeta) \times U(\zeta)}: U(\zeta) \times U(\zeta) \hookrightarrow U(2,2)=U\left(\mathbb{W}_{d} \oplus \mathbb{W}^{d}\right) \subset U(3,3) .
$$

Also for $(x, t) \in N$, we have $\mathfrak{e}\left((x, t), I_{2}\right)=(x \zeta / 2, x, t)$. This implies $\mathfrak{e}\left(N, I_{2}\right) \subset H$. Specifically, the center of $N$ is mapped to the center of $H$ under $\mathfrak{e}$, which explains why I use $T$ to denote both centers of $H$ and $N$.

From Theorem 2.6, we know that

$$
E(g, f, s)=\int_{[U(\zeta)]} \mathbb{E}(\mathfrak{e}(g, u), \mathbb{E}, s) \beta(u) \eta^{-1}(\operatorname{det} u) d u
$$

for $f_{s}(g)=\int_{U(\zeta)(\mathbb{A})} \mathbb{f}_{s}(\mathfrak{e}(g, u)) \beta(u) \eta^{-1}(\operatorname{det} u) d u \in I_{P}^{U\left(I_{\zeta}\right)}(\Pi \otimes \eta, s)$, where $\beta \in V_{\Pi}$. So it is reasonable to infer that $E_{\psi}$ is also the pullback of $\mathbb{E}_{\psi}$. The following proposition is easy to verify.

Proposition 4.10. If $E(g, f, s)$ is defined by (26), then

$$
E_{\psi}(n u, f, s)=\int_{[U(\zeta)]} \mathbb{E}_{\psi}\left(\mathfrak{e}\left(n u, u^{\prime}\right), \mathbb{E}, s\right) \beta\left(u^{\prime}\right) \eta^{-1}\left(\operatorname{det} u^{\prime}\right) d u^{\prime} .
$$

Let us study the relation between $N \cdot U(\zeta)$ and $H \cdot U(2,2)$ induced by the embedding e. $N$ is the Heisenberg group associated to the symplectic group $W$, and $\mathrm{U}(W)=U(\zeta)$, while $H \subset U(3,3)$ is the Heisenberg group associated to $\mathbb{W}=\mathbb{W}_{d}+\mathbb{W}^{d}$, and $\mathrm{U}(\mathbb{W})=U(2,2)$. Notice that

$$
W \stackrel{\mathfrak{e}}{\hookrightarrow} \mathbb{W}, \quad x \mapsto\left(\frac{x \zeta}{z}, x\right) .
$$

At the same time, we can define another embedding of $W^{-}$:

$$
W^{-} \hookrightarrow \mathbb{W}, \quad x \mapsto\left(-\frac{x \zeta}{z}, x\right) .
$$


So, the two embeddings combine to give

$$
\begin{aligned}
W+W^{-} & \sim \\
(x,-x) & \mapsto(x \zeta, 0), \\
(x, x) & \mapsto(0, x) .
\end{aligned}
$$

Since $W$ has the polarization $W=X+Y$, the Weil representation of $\mathrm{U}(2,2)$ can be realized on $S(X+X)$ or on $S\left(\mathbb{W}_{d}\right)$. Recall that we define an intertwining isometry operator $\delta_{\psi}$ between the two representations by (13). It can be applied here with a little revision. Take $\phi \in S(X+X)$, and let $\delta_{\psi}{ }^{\prime}(\phi)(w)=\delta_{\psi}(\phi)\left(w \zeta^{-1}\right)$ be the corresponding function in $S\left(\mathbb{W}_{d}\right)$. If we take $\phi=\phi_{1} \otimes \bar{\phi}_{2}$ for $\phi_{1}, \phi_{2} \in S(X)$, then

$$
\omega_{\chi}\left(\mathfrak{e}\left(u_{1}, u_{2}\right)\right) \delta_{\psi}^{\prime}\left(\phi_{1} \otimes \bar{\phi}_{2}\right)=\delta_{\psi}^{\prime}\left(\omega_{\chi}\left(u_{1}\right) \phi_{1} \otimes \chi\left(\operatorname{det} u_{2}\right) \check{\omega}_{\chi}\left(u_{2}\right) \bar{\phi}_{2}\right) .
$$

It is straightforward to verify that

$$
\theta_{\delta_{\psi}^{\prime}\left(\phi_{1} \otimes \bar{\phi}_{2}\right)}\left(\mathfrak{e}\left(n u_{1}, u_{2}\right)\right)=\chi\left(\operatorname{det} u_{2}\right) \theta_{\phi_{1}}\left(n u_{1}\right) \bar{\theta}_{\phi_{2}}\left(u_{2}\right),
$$

where $\bar{\theta}_{\phi_{2}}\left(u_{2}\right)=\sum_{x \in X(F)} \check{\omega}_{\chi}\left(u_{2}\right) \overline{\phi_{2}}(x)$. Then Proposition 4.10 and Theorem 4.9 give this:

Corollary 4.11. Assume that $\phi_{\mathbb{V}_{k}}=\delta_{\psi}^{\prime}\left(\phi_{1} \otimes \bar{\phi}_{2}\right)$, and $E\left(g, f_{k}\right)$ is defined as the pullback of $\mathbb{E}\left(\cdot, \mathbb{E}_{k}\right)$, where $\mathbb{I}_{k}$ satisfies the conditions of Theorem 4.9. Then

$$
E_{\psi}\left(n u, f_{k}\right)=c_{\mathbb{E}_{k}, \psi} \theta_{\phi_{1}}(n u) \int_{[U(\zeta)]} \beta\left(u^{\prime}\right) \chi \eta^{-1}\left(\operatorname{det} u^{\prime}\right) \mathscr{E}\left(\mathfrak{e}\left(u, u^{\prime}\right), R_{k-1}\right) \bar{\theta}_{\phi_{2}}\left(u^{\prime}\right) d u^{\prime} .
$$

Inner product of $E_{\psi}$ with $\theta_{\varphi}$. As we mentioned, both $E_{\psi}$ and $\theta_{\varphi}$ for $\varphi \in S(X)$ are functions in $C_{\psi}^{\infty}([N U(\zeta)])$. Let $L_{\psi}^{2}([N U(\zeta)])$ be the completion of $C_{\psi}^{\infty}([N U(\zeta)])$ with respect to the inner product

$$
\left\langle\theta, \theta^{\prime}\right\rangle=\int_{[N U(\zeta)]} \theta(r) \overline{\theta^{\prime}}(r) d r .
$$

Treat $E_{\psi}$ and $\theta_{\varphi}$ as elements in $L_{\psi}^{2}([N U(\zeta)])$. Let us calculate $\left\langle E_{\psi}, \theta_{\varphi}\right\rangle$.

Theorem 4.12. In the setting above, we have $\left\langle E_{\psi}\left(n u, f_{k}\right), \theta_{\varphi}(n u)\right\rangle$

$$
=c_{\mathbb{E}_{k}, \psi}\left(\phi_{1}, \varphi\right) \int_{U(\zeta)(\mathbb{A})} R_{k-1}(\mathfrak{e}(u, 1)) d u \int_{[U(\zeta)]} \beta(u) \bar{\theta}_{\phi_{2}}(u) d u,
$$

$\prod_{v \notin S} \int_{U(\zeta)_{v}} R_{k-1, v}(\mathfrak{e}(u, 1)) d u=\frac{L^{S}\left(\eta \chi^{-1}, l-\frac{1}{2}\right) L^{S}\left(\eta \chi^{-1}, l-\frac{3}{2}\right)}{L^{S}\left(\eta_{F} \epsilon_{K / F}, 2 l-1\right) L^{S}\left(\eta_{F}, 2 l-2\right)}$,

where $S$ is the set of places introduced in Theorem 4.9, and $L^{S}$ is the partial $L$-function skipping the factors at $v \in S$. 
(I will discuss the case $v \in S$ in Remark 4.14.)

Proof. We prove the theorem in three steps:

Step 1. $\left\langle E_{\psi}\left(n u, f_{k}\right), \theta_{\varphi}(n u)\right\rangle=c_{\mathbb{t}_{k}, \psi}\left(\phi_{1}, \varphi\right) I\left(E_{\psi}\right)$, where

$$
I\left(E_{\psi}\right)=\int_{[U(\zeta) \times U(\zeta)]} \beta\left(u^{\prime}\right) \chi \eta^{-1}\left(\operatorname{det} u^{\prime}\right) \mathscr{E}\left(\mathfrak{e}\left(u, u^{\prime}\right), R_{k-1}\right) \bar{\theta}_{\phi_{2}}\left(u^{\prime}\right) d u d u^{\prime} .
$$

Step 2. $I\left(E_{\psi}\right)=\int_{U(\zeta)(\mathbb{A})} R_{k-1}(\mathfrak{e}(u, 1)) d u \cdot \int_{[U(\zeta)]} \beta(u) \bar{\theta}_{\phi_{2}}(u) d u$.

Step 3. We show

$$
\int_{U(\zeta)_{v}} R_{k-1, v}(\mathfrak{e}(u, 1)) d u=\frac{L_{v}\left(\eta \chi^{-1}, l-\frac{1}{2}\right) L_{v}\left(\eta \chi^{-1}, l-\frac{3}{2}\right)}{L_{v}\left(\eta_{F} \epsilon_{K / F}, 2 l-1\right) L_{v}\left(\eta_{F}, 2 l-2\right)}
$$

for a finite place $v \notin S$.

The equation in Step 1 is straightforward from Corollary 4.11, because first for $\theta_{\phi_{1}}, \theta_{\varphi} \in L_{\psi}^{2}([N U(\zeta)])$, we have $\left\langle\theta_{\phi_{1}}, \theta_{\varphi}\right\rangle=\left(\phi_{1}, \varphi\right)$; then

$$
\begin{aligned}
& \left\langle E_{\psi}\left(n u, f_{k}\right), \theta_{\varphi}(n u)\right\rangle \\
& \quad=\int_{[N U(\zeta)]} c_{\mathbb{k}_{k}, \psi}\left(\int_{[U(\zeta)]} \beta\left(u^{\prime}\right) \chi \eta^{-1}\left(\operatorname{det} u^{\prime}\right)\right. \\
& \left.\quad \mathscr{E}\left(\mathfrak{e}\left(u, u^{\prime}\right)\right) \bar{\theta}_{\phi_{2}}\left(u^{\prime}\right) d u^{\prime}\right) \theta_{\phi_{1}}(n u) \bar{\theta}_{\varphi}(n u) d n d u \\
& \quad=c_{\mathbb{E}_{k}, \psi}\left(\phi_{1}, \varphi\right) \int_{[U(\zeta) \times U(\zeta)]} \beta\left(u^{\prime}\right) \chi \eta^{-1}\left(\operatorname{det} u^{\prime}\right) \mathscr{E}\left(\mathfrak{e}\left(u, u^{\prime}\right), R_{k-1}\right) \bar{\theta}_{\phi_{2}}\left(u^{\prime}\right) d u d u^{\prime} \\
& \quad=c_{\mathbb{E}_{k}, \psi}\left(\phi_{1}, \varphi\right) I\left(E_{\psi}\right) .
\end{aligned}
$$

To get the expression of $I\left(E_{\psi}\right)$ in Step 2, let us unfold $\mathscr{E}\left(\cdot, R_{k-1}\right)$ :

$$
\begin{aligned}
\mathscr{E}\left(\mathfrak{e}\left(u, u^{\prime}\right), R_{k-1}\right) & =\sum_{\gamma \in \mathscr{P}^{\prime}(F) \backslash U(2,2)(F)} R_{k-1}\left(\gamma \mathfrak{e}\left(u, u^{\prime}\right)\right) \\
& =\sum_{\gamma=\mathfrak{e}\left(u_{1}, u_{2}\right) \in \Delta(U(\zeta))(F) \backslash U(\zeta)(F) \times U(\zeta)(F)} R_{k-1}\left(\gamma \mathfrak{e}\left(u, u^{\prime}\right)\right),
\end{aligned}
$$

where $\Delta(U(\zeta))$ is the image of the diagonal embedding of $\mathrm{U}(\zeta)$ to $\mathrm{U}(\zeta) \times U(\zeta)$. Then,

$$
\begin{aligned}
I\left(E_{\psi}\right) & \\
& =\int_{\Delta(U(\zeta))(F) \backslash U(\zeta)(\mathbb{A}) \times U(\zeta)(\mathbb{A})} \beta\left(u^{\prime}\right) \chi \eta^{-1}\left(\operatorname{det} u^{\prime}\right) \bar{\theta}_{\phi_{2}}\left(u^{\prime}\right) R_{k-1}\left(\mathfrak{e}\left(u, u^{\prime}\right)\right) d u d u^{\prime} \\
& \stackrel{u=u^{\prime} v}{=} \int_{U(\zeta)(\mathbb{A}) \times[U(\zeta)]} \beta\left(u^{\prime}\right) \chi \eta^{-1}\left(\operatorname{det} u^{\prime}\right) \bar{\theta}_{\phi_{2}}\left(u^{\prime}\right) R_{k-1}\left(\mathfrak{e}\left(u^{\prime} v, u^{\prime}\right)\right) d v d u^{\prime} \\
& =\int_{U(\zeta)(\mathbb{A})} R_{k-1}(\mathfrak{e}(u, 1)) d u \cdot \int_{[U(\zeta)]} \beta(u) \bar{\theta}_{\phi_{2}}(u) d u .
\end{aligned}
$$


Now we are left with the calculation of $\int_{U(\zeta)(\mathbb{A})} R_{k-1}(\mathfrak{e}(u, 1)) d u$. It can be written as the product of local integrals:

$$
\int_{U(\zeta)(\mathbb{A})} R_{k-1}(\mathfrak{e}(u, 1)) d u=\prod \int_{v} \int_{U(\zeta)\left(F_{v}\right)} R_{k-1, v}(\mathfrak{e}(u, 1)) d u .
$$

In the following computations, we drop the subscript $v$ if this does not cause confusion.

First, $v=\infty$. Now $R_{k-1, \infty}$ is defined by the automorphy factor, namely $R_{k-1, \infty}\left(g^{\prime}\right)=j\left(g^{\prime}, i^{\prime}\right)^{-k+1}$, and $\mathrm{U}(\zeta)\left(F_{\infty}\right)$ is isomorphic to copies of the compact group $\mathrm{U}(2)(\mathbb{R})$. By Proposition $4.4, R_{k-1}(\mathfrak{e}(u, 1))=1$. So,

$$
\int_{U(\zeta)\left(F_{\infty}\right)} R_{k-1, \infty}(\mathfrak{e}(u, 1)) d u=\operatorname{vol}\left(U(\zeta)\left(F_{\infty}\right)\right)
$$

Second, $v$ splits in $F$. Then $\mathrm{U}(\zeta)\left(F_{v}\right) \simeq \mathrm{GL}_{2}\left(F_{v}\right)$. Assume $R_{k-1, v}$ is normalized and spherical. Now, $K=F+F, \eta(a, b)=\eta_{1}(a) \eta_{2}(b)$ for $(a, b) \in K$, and similarly for $\chi$. Since $\left.\chi\right|_{\mathbb{A}^{\times}}=\epsilon_{K / F}$, we have $\chi_{1} \chi_{2}=1$. Let us take the Godement section representation of $R_{k-1}$ :

$$
\begin{aligned}
& L\left(\eta_{F}, 2 l-1\right) L\left(\eta_{F}, 2 l-2\right) R_{k-1}(g) \\
& \quad=\eta_{1} \chi_{1}^{-1}(\operatorname{det} g)|\operatorname{det} g|^{l-\frac{1}{2}} \int_{\mathrm{GL}_{2}(F)} \Phi_{M_{2 \times 4}\left(\mathcal{O}_{F}\right)}((0, X) g) \eta_{1} \eta_{2}(\operatorname{det} X)|\operatorname{det} X|^{2 l-1} d X,
\end{aligned}
$$

where $\Phi_{M_{2 \times 4}\left(\mathscr{O}_{F}\right)}$ is the characteristic function of $M_{2 \times 4}\left(\mathscr{O}_{F}\right)$. Then

$$
\begin{aligned}
& L\left(\eta_{F}, 2 l-1\right) L\left(\eta_{F}, 2 l-2\right) R_{k-1}(\mathfrak{e}(u, 1)) \\
& \quad=\eta_{1} \chi_{1}^{-1}(\operatorname{det} u)|\operatorname{det} u|^{l-\frac{1}{2}} \int_{\mathrm{GL}_{2}(F)} \Phi_{M_{2 \times 4}\left(\mathbb{O}_{F}\right)}(X, X u) \eta_{1} \eta_{2}(\operatorname{det} X)|\operatorname{det} X|^{2 l-1} d X .
\end{aligned}
$$

So,

$$
\begin{aligned}
& L\left(\eta_{F}, 2 l-1\right) L\left(\eta_{F}, 2 l-2\right) \int_{U(\zeta)} R_{k-1}(\mathfrak{e}(u, 1)) d u \\
& \quad=L\left(\eta_{F}, 2 l-1\right) L\left(\eta_{F}, 2 l-2\right) \int_{\mathrm{GL}_{2}(F)} R_{k-1}(\mathfrak{e}(u, 1)) d u \\
& \quad=\int_{\mathrm{GL}_{2}(F) \times \mathrm{GL}_{2}(F)} \eta_{1} \chi_{1}^{-1}(\operatorname{det} u)|\operatorname{det} u|^{l-\frac{1}{2}} \\
& \stackrel{Y=X u}{=} \int_{\mathrm{GL}_{2}} \eta_{1} \chi_{1}^{-1}(\operatorname{det} Y)|\operatorname{det} Y|^{l-\frac{1}{2}} \Phi_{\mathbb{O}_{F}^{2}}(Y) d Y \\
& \quad \int_{\mathrm{GL}_{2}} \eta_{2} \chi_{1}(\operatorname{det} X)|\operatorname{det} X|^{l-\frac{1}{2}} \Phi_{\mathbb{O}_{F}^{2}}(X) d X
\end{aligned}
$$


which is equal to

$$
L\left(\eta \chi^{-1}, l-\frac{1}{2}\right) L\left(\eta \chi^{-1}, l-\frac{3}{2}\right) .
$$

Then we have

$$
\int_{\mathrm{GL}_{2}(F)} R_{k-1}(\mathfrak{e}(u, 1)) d u=\frac{L\left(\eta \chi^{-1}, l-\frac{1}{2}\right) L\left(\eta \chi^{-1}, l-\frac{3}{2}\right)}{L\left(\eta_{F}, 2 l-1\right) L\left(\eta_{F}, 2 l-2\right)} .
$$

First, $R_{k-1, v}$ is normalized and spherical, and $\mathrm{U}(\zeta)\left(F_{v}\right)$ is quasisplit over $F_{v}$. So $\mathrm{U}(\zeta) \simeq U(1,1)$. The embedding $\mathfrak{e}$ of $\mathrm{U}(1,1) \times U(1,1)$ to $\mathrm{U}(2,2)$ is inherited from the global definition of $\left.\mathfrak{e}\right|_{U(\zeta) \times U(\zeta)}$.

Take the local Iwasawa decomposition $\mathrm{U}(1,1)=P K=N M K$, where $N, M$ and $K$ are the unipotent radical, Levi part and maximal open compact subgroup, respectively. Then $K=U(1,1)\left(\mathscr{O}_{F}\right)$, and $\mathfrak{e}(K, K) \subset \mathscr{K}^{\prime}$, which is the maximal compact subgroup of $\mathrm{U}(2,2)$. Let $u=n m k$; then $d u=\delta_{P}^{-1}(m) d n d m d k$, where $\delta_{P}$ is the modular character on $P$. If $m=\left(\bar{\alpha}_{\alpha}^{-1}\right)$, then $\delta_{P}(m)^{-1}=|\alpha|_{K}$. Let

$$
w_{13}=\left(\begin{array}{ccc} 
& 1 & \\
& & \\
& & \\
& & 1
\end{array}\right)
$$

then

$$
\begin{aligned}
\int_{U(\zeta)} R_{k-1}(\mathfrak{e}(u, 1)) d u & =\int_{N M} R_{k-1}(\mathfrak{e}(n m, 1)) \delta_{P}^{-1}(m) d n d m \\
& =\int_{M} \int_{F} R_{k-1}\left(w_{13}\left(\begin{array}{ccc}
1 & & x \\
& 1 & \\
& & 1
\end{array}\right) w_{13}^{-1} \mathfrak{e}(m, 1)\right) \delta_{P}^{-1}(m) d x d m .
\end{aligned}
$$

Consider such a function on $\mathrm{U}(2,2)$ :

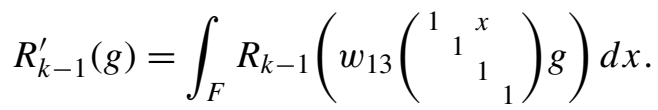

We have

$$
\int_{U(\zeta)} R_{k-1}(\mathfrak{e}(u, 1)) d u=\int_{M} R_{k-1}^{\prime}\left(w_{13}^{-1} \mathfrak{e}(m, 1)\right) \delta_{P}^{-1}(m) d m .
$$

Recall that $\mathrm{U}(2,2)=U(\mathbb{W})=U\left(\mathbb{W}_{d}+\mathbb{W}^{d}\right)$. If we have

$$
\mathbb{W}_{d}+\mathbb{W}^{d}=K e_{1}+K e_{2}+K f_{1}+K f_{2},
$$

then a parabolic subgroup $P^{\prime}$ fixing both $\mathbb{W}_{d}$ and $K e_{2}$ can be defined. An element $p^{\prime} \in P^{\prime}$ looks like

$$
\left(\begin{array}{cccc}
a & & & d \\
b & c & e & f \\
& & a^{\prime} & b^{\prime} \\
& & & c^{\prime}
\end{array}\right) .
$$


Lemma 4.13. $R_{k-1}^{\prime}$ is in the space of the induced representations from $P^{\prime}$, so that

$$
R_{k-1}^{\prime}\left(p^{\prime} g k^{\prime}\right)=\eta \chi^{-1}\left(a^{\prime} c\right)\left|a^{\prime}\right|_{K}^{l-\frac{3}{2}}|c|_{K}^{l-\frac{1}{2}} R_{k-1}^{\prime}(g)
$$

for $p^{\prime} \in P^{\prime}, k^{\prime} \in \mathscr{K}^{\prime}$ and $g \in U(2,2)$.

This lemma can be proved by direct calculations, which I will skip here. Applying the lemma, we see $R_{k-1}^{\prime}$ has a Godement section representation as follows:

$$
\begin{aligned}
& \frac{L\left(\eta_{F} \epsilon_{K / F}, 2 l-1\right)}{L\left(\eta_{F} \epsilon_{K / F}, 2 l-2\right)} R_{k-1}^{\prime}(g) \\
& =c \int_{\mathrm{GL}_{2}(K)} \Phi_{M_{2 \times 4}\left(\mathbb{O}_{K}\right)}((0, X) g) \eta^{-1} \chi(\operatorname{det} X)|\operatorname{det} X|_{K}^{-l+\frac{3}{2}} d X . \\
& \quad \cdot \int_{K^{\times}} \Phi_{\bigcirc_{K}^{4}}((0,0,0, Z) g) \eta \chi^{-1}\left(|Z|_{K}\right)|Z|_{K}^{2 l-2} d Z,
\end{aligned}
$$

where the normalizing constant $c$ satisfies

$$
\begin{aligned}
& 1=c \int_{\mathrm{GL}_{2}(K)} \Phi_{M_{2 \times 2}\left(\mathbb{O}_{K}\right)}(X) \eta^{-1} \chi(\operatorname{det} X)|\operatorname{det} X|_{K}^{-l+\frac{3}{2}} d X \\
& \cdot \int_{K^{\times}} \Phi_{\bigcirc_{K}}(Z) \eta \chi^{-1}\left(|Z|_{K}\right)|Z|_{K}^{2 l-2} d Z
\end{aligned}
$$

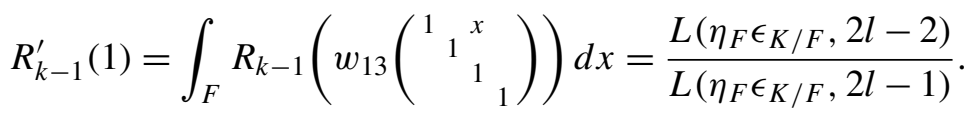

Let $g=w_{13}^{-1} \mathfrak{e}(m, 1)=w_{13}^{-1} \mathfrak{e}\left(\left(\bar{\alpha}_{\alpha}^{-1}\right), 1\right)$. It can be verified that $R_{k-1}^{\prime}\left(w_{13}^{-1} \mathfrak{e}(m, 1)\right)$ $=\frac{\eta \chi^{-1}(\alpha)|\alpha|_{K}^{l-\frac{3}{2}}}{L\left(\eta_{F} \epsilon_{K / F}, 2 l-1\right) L\left(\eta_{F}, 2 l-2\right)} \int_{K^{\times}} \Phi_{\bigcirc_{K}}(Z) \Phi_{\mathscr{O}_{K}}(Z \alpha) \eta \chi^{-1}\left(|Z|_{K}\right)|Z|_{K}^{2 l-2} d Z$.

Substituting the expression of $R_{k-1}^{\prime}\left(w_{13} \mathfrak{e}(m, 1)\right)$ in (27), we have

$$
\begin{aligned}
& \int_{U(\zeta)} R_{k-1}(\mathfrak{e}(u, 1)) d u \\
&=\int_{M} R_{k-1}^{\prime}\left(w_{13}^{-1} \mathfrak{e}(m, 1)\right) \delta_{P}^{-1}(m) d m \\
&=\frac{1}{L\left(\eta_{F} \epsilon_{K / F}, 2 l-1\right) L\left(\eta_{F}, 2 l-2\right)} \\
& \cdot \int_{K^{\times} \times K^{\times}} \eta \chi^{-1}(\alpha)|\alpha|_{K}^{l-\frac{1}{2}} \Phi_{\bigcirc_{K}}(Z) \Phi_{\bigcirc_{K}}(Z \alpha) \eta \chi^{-1}\left(|Z|_{K}\right)|Z|_{K}^{2 l-2} d Z d \alpha \\
&= \frac{L\left(\eta \chi^{-1}, l-\frac{1}{2}\right) L\left(\eta \chi^{-1}, l-\frac{3}{2}\right)}{L\left(\eta_{F} \epsilon_{K / F}, 2 l-1\right) L\left(\eta_{F}, 2 l-2\right)} .
\end{aligned}
$$


Remark 4.14. In Theorem 4.12, $\int_{U(\zeta)\left(F_{v}\right)} R_{k-1, v}(\mathfrak{e}(u, 1)) d u$ was not explicitly calculated when $v \in S$, because we need more assumptions and things become more technical. Let me put it here. When $v \in S, R_{k-1, v}$ is supported in the big cell associated to a characteristic function $\Phi^{\prime}$ on $\operatorname{Her}_{2}\left(F_{v}\right)$. (One can refer to the section about nonspherical $\mathbb{\llbracket}_{k}$, especially Lemma 4.8.)

Skip the subindex $v$ now. Fix an integral ideal $\mathfrak{c}$ of $\mathscr{O}_{K}$ so that $\eta \chi^{-1}(1+\mathfrak{c})=1$. Then pick a totally imaginary element $\delta \in \mathcal{O}_{K}$ satisfying $1+\delta \in \mathcal{O}_{K}^{\times}$. Assume that

$$
\operatorname{supp} \Phi^{\prime}=\operatorname{Her}_{2}(F) \cap \delta \zeta^{-1}\left(2 I_{2}+2 \mathfrak{c} \operatorname{GL}_{2}\left(\mathscr{O}_{K}\right)\right) .
$$

Define $D_{1}(\mathfrak{c})$, a subset of $\mathrm{U}(\zeta)$, by

$$
D_{1}(\mathfrak{c})=\left\{u \in U(\zeta) \mid u=\left(\begin{array}{ll}
a & b \\
c & d
\end{array}\right), a, d \in-\frac{1+\delta}{1-\delta}+\delta \mathfrak{c}, b, c \in \delta \mathfrak{c}\right\} .
$$

Lemma 4.15. Let $u \in U(\zeta)$. Then

$$
\mathfrak{e}(u, 1) \in \operatorname{supp} R_{k-1} \Longleftrightarrow u \in D_{1}(\mathfrak{c}) .
$$

Proof. First notice that for a matrix in $\mathrm{U}(2,2)$,

$$
\left(\begin{array}{ll}
a & b \\
c & d
\end{array}\right) \in \operatorname{supp} R_{k-1} \Longleftrightarrow c^{-1} d \in \operatorname{supp} \Phi^{\prime} .
$$

By the definition of the embedding $\mathfrak{e}$ in (8),

$$
\mathfrak{e}(u, 1)=\left(\begin{array}{cc}
\left(\zeta^{-1}\left(u+I_{2}\right) \zeta\right) / 2 & \zeta^{-1}\left(u-I_{2}\right) \\
\left(\left(u-I_{2}\right) \zeta\right) / 4 & \left(u+I_{2}\right) / 2
\end{array}\right) .
$$

So

$$
\begin{aligned}
\mathfrak{e}(u, 1) \in \operatorname{supp} R_{k-1} & \Longleftrightarrow 2 \zeta^{-1}\left(u-I_{2}\right)^{-1}\left(u+I_{2}\right) \in \operatorname{supp} \Phi^{\prime} \\
& \Longleftrightarrow u \in D_{1}(\mathfrak{c}) .
\end{aligned}
$$

Then we get

$$
R_{k-1}(\mathfrak{e}(u, 1))= \begin{cases}\eta \chi^{-1}(\delta) & \text { if } u \in D_{1}(\mathfrak{c}) \\ 0 & \text { otherwise }\end{cases}
$$

The integral

$$
\int_{U(\zeta)} R_{k-1}(\mathfrak{e}(u, 1)) d u=\eta \chi^{-1}(\delta) \operatorname{vol}\left(D_{1}(\mathfrak{c})\right)
$$

As we see from Theorem 4.12, $\left\langle E_{\psi}, \theta_{\varphi}\right\rangle$ equals the product of an explicit constant and the integral $\int_{[U(\zeta)]} \beta(u) \bar{\theta}_{\phi_{2}}(u) d u$. Using the discussion of Section $3 \mathrm{C}$, I will show that this integral can be interpreted by the theta lifting from $\mathrm{U}(\zeta)$ to $\mathrm{U}(1)$.

Recall that $N$ is the unipotent radical of the parabolic subgroup $P$ of $\mathrm{U}\left(I_{\zeta}\right)$. We take $N$ as the Heisenberg group associated to the 4-dimensional symplectic space $W$ over $F$. On $W$, the alternating pairing is defined as $\left\langle x_{1}, x_{2}\right\rangle=x_{1} \zeta x_{2}^{*}-x_{2} \zeta x_{1}^{*}$, 
while, as a $K$-vector space, $W=V_{1} \otimes V_{2}$, where $V_{1}$ and $V_{2}$ are Hermitian vector spaces with dimensions 2 and 1 over $K$, respectively. The skew-Hermitian form on $V_{1}$ is $(x, y)_{1}=x \zeta y^{*}$. The Hermitian form on $V_{2}$ is defined as $(x, y)_{2}=x \bar{y}$. So, we immediately have the reductive pair $\left(U\left(V_{1}\right), U\left(V_{2}\right)\right)$ in $\operatorname{Sp}(W)$ with $\mathrm{U}\left(V_{1}\right) \simeq U(\zeta)$, and $\mathrm{U}\left(V_{2}\right) \simeq U(1)$.

Consider the theta lifting

$$
\theta_{\phi_{2}}^{\beta}(u)=\int_{U(\zeta)} \theta_{\phi_{2}}(g, u) \beta(g) d g
$$

from $\mathrm{U}(\zeta)$ to $\mathrm{U}(1)$. In Section $3 \mathrm{C}$, I give the definition of a theta lifting and explain the way to compute $\left|\theta_{\phi_{2}}^{\beta}(1)\right|^{2}$ using the Siegel-Weil formula. If we assume that the representation $\Pi$ on $\mathrm{U}(\zeta)$ is self-dual, then $V_{\Pi} \simeq V_{\check{\Pi}}$, and

$$
\left|\int_{[U(\zeta)]} \beta(u) \bar{\theta}_{\phi_{2}}(u) d u\right|^{2}=\left|\theta_{\phi_{2}}^{\beta}(1)\right|^{2}
$$

Proposition 3.7 and (18) together imply:

Corollary 4.16. $\left|\theta_{\phi_{2}}^{\beta}(1)\right|^{2}=\left.\frac{1}{2} \int_{U(\zeta)(\mathbb{A})} f_{\delta_{\psi}\left(\phi_{2} \otimes \bar{\phi}_{2}\right), s}(\mathfrak{e}(g, 1))\langle\Pi(g) \beta, \beta\rangle d g\right|_{s=-\frac{1}{2}}$.

Remark 4.17. The integral in the corollary above should be nonzero if $\phi_{2}$ is chosen properly, because the theta lifting of $\Pi$ should define a nonzero representation space of $\mathrm{U}(1)$. This is a special case in [Li 1992]. The nonzero result is crucial for our application.

Remark 4.18. If $\phi_{2, v}$ is a standard characteristic function, then $\delta_{\psi}\left(\phi_{2} \otimes \bar{\phi}_{2}\right)$ is also a standard characteristic function, and $f_{\delta_{\psi}\left(\phi_{2} \otimes \bar{\phi}_{2}\right), s, v}$ is normalized spherical.

Proposition 4.19. If $\Pi_{v}$ is an unramified representation with a spherical vector $\beta$, $\mathfrak{f}_{s, v}$ is the unique $\mathrm{U}(2,2)\left(\mathcal{O}_{F_{v}}\right)$-invariant section in $I_{\mathscr{P}^{\prime}}^{U(2,2)}(\chi, s)_{v}$ and $\mathfrak{f}_{s, v}(1)=1$, then

$$
\int_{U(\zeta)_{v}} \mathfrak{f}_{s, v}(\mathfrak{e}(u, 1))\langle\Pi(u) \beta, \beta\rangle_{v} d u=\frac{L\left(\Pi_{v}, \chi_{v}, s+\frac{1}{2}\right)}{L_{v}\left(\epsilon_{K / F}, 2 s+2\right) L_{v}(1,2 s+1),}
$$

where $L_{v}(1, \cdot)$ is the Zeta function of the local field $F_{v}$.

Remark 4.20. This type of integral was considered by Piatetski-Shapiro and Rallis in many cases. Similar calculations have been done in [Li 1992]. In the $(U(1), U(1))$ case, Yang [1997] had explicit formulas.

Proof. Let me calculate the integral above in the case when $\mathrm{U}(\zeta)_{v} \simeq \mathrm{GL}_{2}\left(F_{v}\right)$. The computations at other types of unramified places are skipped here. Now we omit the subindex $v$. 
Denote the matrix coefficient of $\Pi$ by $w_{\Pi}$. Let $\left.\chi\right|_{F \times F}=\left(\chi_{1}, \chi_{2}\right)$. Using the Godement section of $\mathfrak{f}_{s}$, we have

$$
\begin{aligned}
& \frac{1}{L\left(\epsilon_{K / F}, 2 s+2\right) L\left(\epsilon_{K / F}, 2 s+1\right)} \int_{U(\zeta)} \mathfrak{f}_{s}(\mathfrak{e}(u, 1))\langle\Pi(u) \beta, \beta\rangle d u \\
& =\int_{\mathrm{GL}_{2}(F)} \chi_{1}(\operatorname{det} u)|\operatorname{det} u|^{s+1} \\
& \cdot \int_{\mathrm{GL}_{2}(F)} \Phi_{M_{2 \times 4}\left(\mathbb{O}_{F}\right)}((0, Z) \mathfrak{e}(u, 1)) \epsilon_{K / F}(\operatorname{det} Z)|\operatorname{det} Z|^{2 s+2} w_{\Pi}(u) d u d Z \\
& =\int_{\mathrm{GL}_{2}(F) \times \mathrm{GL}_{2}(F)} \Phi_{M_{2 \times 4}\left(\mathrm{O}_{F}\right)}(Z, Z u) \chi_{1}(\operatorname{det} u)|\operatorname{det} u|^{s+1} \\
& \cdot \epsilon_{K / F}(\operatorname{det} Z)|\operatorname{det} Z|^{2 s+2} w_{\Pi}(u) d u d Z \\
& \stackrel{Y=Z u}{=} \int_{\mathrm{GL}_{2}(F) \times \mathrm{GL}_{2}(F)} \Phi_{M_{2 \times 4}\left(\mathrm{O}_{F}\right)}(Y, Z) \chi_{1}(\operatorname{det} Y)|\operatorname{det} Y|^{s+1} \\
& \cdot \chi_{2}(\operatorname{det} Z)|\operatorname{det} Z|^{s+1} w_{\Pi}\left(Z^{-1} Y\right) d Y d Z \text {. }
\end{aligned}
$$

Let $\mathrm{GL}_{2}(F)=B K$, where $B$ consists of upper triangular matrices and $K=\mathrm{GL}_{2}\left(\mathscr{O}_{F}\right)$. The matrix coefficient $w_{\Pi}$ is a zonal spherical function and satisfies

$$
\int_{K} w_{\Pi}(X k Y) d k=w_{\Pi}(X) w_{\Pi}(Y) \quad \text { for } X, Y \in \mathrm{GL}_{2}(F)
$$

Lemma 4.21. The expression in (29) equals the product of

$$
\int_{\mathrm{GL}_{2}(F)} \Phi_{M_{2}\left(\mathbb{O}_{F}\right)}(Y) \chi_{1}(Y)|\operatorname{det} Y|^{s+1} w_{\Pi}(Y) d Y
$$

and

$$
\int_{\mathrm{GL}_{2}(F)} \Phi_{M_{2}\left(\mathbb{O}_{F}\right)}(X) \chi_{2}(X)|\operatorname{det} X|^{s+1} w_{\Pi}(X) d X .
$$

Proof. First, it is obvious that

$$
\text { (29) }=\int_{\mathrm{GL}_{2}(F) \times \mathrm{GL}_{2}(F) \times K} \Phi_{M_{2 \times 4}\left(\mathbb{O}_{F}\right)}(k Y, Z) \chi_{1}(\operatorname{det} k Y)|\operatorname{det} k Y|^{s+1}
$$

$\cdot \chi_{2}(\operatorname{det} Z)|\operatorname{det} Z|^{s+1} w_{\Pi}\left(Z^{-1} Y\right) d Y d Z d k$. 
Then, substitute $k Y$ by $Y^{\prime}$ :

$$
\begin{aligned}
&(30)= \int_{\mathrm{GL}_{2}(F) \times \mathrm{GL}_{2}(F)} \Phi_{M_{2 \times 4}\left(\mathrm{O}_{F}\right)}\left(Y^{\prime}, Z\right) \chi_{1}\left(\operatorname{det} Y^{\prime}\right)\left|\operatorname{det} Y^{\prime}\right|^{s+1} \chi_{2}(\operatorname{det} Z)|\operatorname{det} Z|^{s+1} \\
& \cdot \int_{K} w_{\Pi}\left(Z^{-1} k^{-1} Y^{\prime}\right) d k d Y^{\prime} d Z \\
&=\int_{\mathrm{GL}_{2}(F)} \Phi_{M_{2}\left(\mathbb{O}_{F}\right)}(Y) \chi_{1}(Y)|\operatorname{det} Y|^{s+1} w_{\Pi}(Y) d Y \\
& \cdot \int_{\mathrm{GL}_{2}(F)} \Phi_{M_{2}\left(\mathbb{O}_{F}\right)}(Z) \chi_{2}(Z)|\operatorname{det} Z|^{s+1} w_{\Pi}\left(Z^{-1}\right) d Z .
\end{aligned}
$$

So this proves Lemma 4.21.

Let

$$
Z\left(\Phi_{M_{2}\left(\mathbb{O}_{F}\right)}, \chi_{1} \otimes w_{\Pi}, s+1\right)=\int_{\mathrm{GL}_{2}(F)} \Phi_{M_{2}\left(\mathscr{C}_{F}\right)}(Y) \chi_{1}(Y)|\operatorname{det} Y|^{s+1} w_{\Pi}(Y) d Y .
$$

Zeta integrals such as this are discussed in [Godement and Jacquet 1972].

Lemma 4.22. If $\Pi=\pi\left(\mu_{1}, \mu_{2}\right)$, then

$$
Z\left(\Phi_{M_{2}\left(\Theta_{F}\right)}, \chi_{1} \otimes w_{\Pi}, s+1\right)=L\left(\chi_{1} \mu_{1}, s+\frac{1}{2}\right) L\left(\chi_{2} \mu_{2}, s+\frac{1}{2}\right) .
$$

Proof. Let $Y=p k$, for $p=\left(\begin{array}{cc}a_{1} & b \\ a_{2}\end{array}\right) \in B$ and $k \in K$. Then $d Y=\left(1 /\left|a_{1}\right|\right) d b d a_{1} d a_{2}$. $w_{\Pi}(Y)=w_{\Pi}(p)=\mu_{1}\left(a_{1}\right) \mu_{2}\left(a_{2}\right)\left(\left|a_{1}\right| /\left|a_{2}\right|\right)^{\frac{1}{2}}$. So,

$$
\begin{aligned}
Z\left(\Phi_{M_{2}\left(\mathscr{O}_{F}\right)}, \chi_{1} \otimes w_{\Pi}, s+1\right) & =\int_{\mathscr{O}_{F} \times \mathbb{O}_{F}} \chi_{1} \mu_{1}\left(a_{1}\right)\left|a_{1}\right|^{s+\frac{1}{2}} \chi_{1} \mu_{2}\left(a_{2}\right)\left|a_{2}\right|^{s+\frac{1}{2}} d a_{1} d a_{2} \\
& =L\left(\chi_{1} \mu_{1}, s+\frac{1}{2}\right) L\left(\chi_{2} \mu_{2}, s+\frac{1}{2}\right) .
\end{aligned}
$$

By Lemmas 4.21 and 4.22, we have

$$
\int_{U(\zeta)} \mathfrak{f}_{s}(\mathfrak{e}(u, 1))\langle\Pi(u) \beta, \beta\rangle d u=\frac{L\left(\Pi, \chi, s+\frac{1}{2}\right)}{L\left(\epsilon_{K / F}, 2 s+2\right) L\left(\epsilon_{K / F}, 2 s+1\right)} .
$$

And notice that in this case, $\epsilon_{K / F}(\varpi)=1$ for the prime element $\varpi$ in $F$. This proves Proposition 4.19 when $\mathrm{U}(\zeta) \simeq \mathrm{GL}_{2}(F)$.

Next, let me explain the local integrals of $\left|\theta_{\phi_{2}}^{\beta}(1)\right|^{2}$ at archimedean places and ramified finite places. At archimedean places, $f_{\delta_{\psi}\left(\phi_{2} \otimes \bar{\phi}_{2}\right), s, \infty}$ is $\mathrm{U}(\zeta)_{\infty} \times U(\zeta)_{\infty}$ invariant, and $\Pi_{\infty}$ is one-dimensional. Apparently,

$$
\int_{U(\zeta)_{\infty}} f_{\delta_{\psi}\left(\phi_{2} \otimes \bar{\phi}_{2}\right), s, \infty}(\mathfrak{e}(g, 1))\langle\Pi(g) \beta, \beta\rangle_{\infty} d g=\operatorname{vol}\left(U(\zeta)_{\infty}\right) \neq 0 .
$$


As for finite places, first we are always allowed to take the standard characteristic function $\phi_{2, v}$ when $\Pi_{v}$ is unramified. This means if $\Pi_{v}$ is unramified, we can guarantee $f_{\delta_{\psi}\left(\phi_{2} \otimes \bar{\phi}_{2}\right), s, v}$ is spherical.

If $\Pi_{v}$ is ramified at a finite place $v$, let $D_{v} \subset U(\zeta)_{v}$ be a compact open subgroup fixing $\Pi_{v}$. Especially, when $\Pi_{v}=I\left(\mu_{1}, \mu_{2}\right)$ is a ramified principal series associated to the local characters $\mu_{1}$ and $\mu_{2}$, we assume that $\mu_{1}$ is ramified and $\mu_{2}$ is unramified. Then $D_{v}=\left\{g \in U(\zeta)_{v} \mid g=\left(\begin{array}{ll}a & b \\ c & d\end{array}\right), a \in 1+\mathfrak{m}, b, d \in \mathcal{O}_{K_{v}}, c \in \mathfrak{m}\right\}$, where $\mathfrak{m}$ is the conductor of $\mu_{1}$. We can choose $\phi_{2, v}$ so that $f_{\delta_{\psi}\left(\phi_{2} \otimes \bar{\phi}_{2}\right), s, v}(\mathfrak{e}(g, 1))=\Phi_{D_{v}}(g)$, where $\Phi_{D_{v}}$ is the characteristic function with the support in $D_{v}$. For instance, if $v$ splits, $\mathrm{U}(\zeta)_{v} \simeq \mathrm{GL}_{2}\left(F_{v}\right)$. Then $\mu_{1}$ and $\mu_{2}$ are both characters of $F_{v}$. We can take $\phi_{2}$ such that

$$
\phi_{2}(x)= \begin{cases}1 & \text { if } x \in\left(\begin{array}{l}
1 \\
0
\end{array}\right)+\mathfrak{m} \mathbb{O}_{F_{v}}^{2} \\
0 & \text { otherwise }\end{cases}
$$

In this way,

$$
\int_{U(\zeta)_{v}} f_{\delta_{\psi}\left(\phi_{2} \otimes \bar{\phi}_{2}\right), s, v}(\mathfrak{e}(g, 1))\langle\Pi(g) \beta, \beta\rangle_{v} d g=\operatorname{vol}\left(D_{v}\right) \neq 0
$$

\section{Applications to Eisenstein series nonvanishing modulo $p$}

Section 4 calculated the Fourier-Jacobi coefficients of two Eisenstein series. One is the holomorphic Siegel-Eisenstein series $\mathbb{E}_{k}(\cdot, \eta)$ on $\mathrm{U}(3,3)$. The other is the holomorphic Eisenstein series $E_{k}(\cdot, \Pi, \eta)$ on $\mathrm{U}\left(I_{\zeta}\right)$ that is from the pullback of $\mathbb{E}_{k}(\cdot, \eta)$. From now on, we fix a prime $p$, so that every prime in $F$ above $p$ is unramified for both Eisenstein series. In this section, we will briefly discuss how to apply the computations of Section 4 to look for an Eisenstein series on $U(3,1)$ that does not vanish modulo $p$.

Remark 5.1. (1) Recall that $\mathbb{E}_{k}(\cdot, \eta)$ is defined from the section $\mathbb{E}_{k}$ that is spherical outside $S$ (for the definition of $S$, one may refer to Theorem 4.9). In the application, $S$ is usually taken to be the set of places where the given data (for instance the number fields $F$ and $K$, the characters $\eta$ and $\chi$, the representation $\Pi$ and so on) are ramified. Notice that we can assume that all the data at $p$ are unramified. So $p \notin S$. In other words, $\mathbb{f}_{k}$ is spherical at $p$.

(2) $\mathbb{E}_{k}(\cdot, \eta)$ can be normalized to become a $p$-integral Eisenstein series $\mathbb{E}^{\text {int }}$. About this claim, one can refer to (3.3.5.3) and Remark (3.3.5.5) in [Harris et al. 2006]. The normalizing factor is given in (3.3.5.1). In fact, the situation here is simpler than [ibid.], because $\mathbb{E}_{k}(\cdot, \eta)$ is unramified at $p$. 
(3) The pullback of $\mathbb{E}^{\mathrm{int}}$ denoted by $\mathbb{E}_{\mathrm{pb}}^{\mathrm{int}}$ is an Eisenstein series on $\mathrm{U}\left(I_{\zeta}\right)$. It only differs by a constant with $E(\cdot, \Pi, \eta)$. Define

$$
E^{\mathrm{int}}=\frac{\mathbb{E}_{\mathrm{pb}}^{\mathrm{int}}}{\Omega_{K}^{k}},
$$

where $\Omega_{K}$ is the CM-period of $K$ and it is well-defined up to $\overline{\mathbb{Z}}_{p}^{\times}$.

Lemma 5.2. $E^{\text {int }}$ is a p-integral holomorphic Eisenstein series.

About $\Omega_{K}$ and this lemma, one can refer to [Hsieh 2011b, Section 7.2].

As mentioned in the introduction, one of the motivations of this paper is to provide a possible way to argue nonvanishing modulo $p$ of the Eisenstein series on the unitary group $\mathrm{U}(3,1)$ used in the Iwasawa theory through the calculation of its Fourier-Jacobi coefficients. For the discussion on this topic, let me assume that $F=\mathbb{Q}$ and that the imaginary quadratic extension $K / \mathbb{Q}$ splits at $p$.

Following the idea of Skinner and Urban [Skinner and Urban 2012; Urban 2006] to show one divisibility of the main conjecture for $\mathrm{GL}_{2} \times K^{\times}$by the method of Eisenstein congruence on $\mathrm{U}(3,1)$, a Hida family of holomorphic Eisenstein series $\mathscr{E}$ ord on $\mathrm{U}(3,1)$ is constructed so that its constant terms at all cusps are divisible by the $p$-adic $L$-function of $\mathrm{GL}_{2} \times K^{\times}$. Suppose $\mathscr{E}$ ord is defined over a two-variable Iwasawa algebra $\Lambda$ (refer to Conjecture 1.1), and denote by $\mathfrak{m}_{\Lambda}$ the maximal ideal of $\Lambda$. It is required that $\mathscr{E}^{\circ}$ ord $\not \equiv 0\left(\bmod \mathfrak{m}_{\Lambda}\right)$. Since $\mathscr{E}$ ord is obtained by interpolating a $p$-ordinary holomorphic Eisenstein series $E^{\text {ord }}$, we have:

Lemma 5.3. If $E^{\text {ord }} \not \equiv 0\left(\bmod \mathfrak{m}_{p}\right)$, then $\mathscr{E}$ ord $\not \equiv 0\left(\bmod \mathfrak{m}_{\Lambda}\right)$, where $\mathfrak{m}_{p}$ is the maximal ideal of $\overline{\mathbb{Z}}_{(p)}$ induced by $i: \mathbb{C} \rightarrow \mathbb{C}_{p}$.

So it is enough to show $E^{\text {ord }}$ does not vanish modulo $\mathfrak{m}_{p}$. For the strict definition and construction of $E^{\text {ord }}$, see [Hsieh 2011b]. Although $E^{\text {ord }}$ and $E^{\text {int }}$ are both $p$-integral holomorphic Eisenstein series, they are not the same, because we assume $E^{\text {int }}$ is unramified at $p$, but $E^{\text {ord }}$ is ramified at $p$. So in order to apply the computation of Fourier-Jacobi coefficients of $E^{\text {int }}$ in Section 4, two points will be addressed in this section. The first is to relate $E^{\text {ord }}$ used in the proof of the main conjecture to $E^{\text {int }}$. The second is to give a strategy of showing nonvanishing modulo $p$ of $E^{\text {int }}$.

At $p, \mathrm{U}(\zeta)\left(\mathbb{Q}_{p}\right) \simeq \mathrm{GL}_{2}\left(\mathbb{Q}_{p}\right)$. In [Hsieh 2011b], the representation $\Pi$ of $\mathrm{U}(\zeta)$ is chosen so that the local representation $\Pi_{p}$ of $\mathrm{GL}_{2}\left(\mathbb{Q}_{p}\right)$ is ordinary; then $\Pi_{p}$ must be of the type $\pi\left(\mu_{1}, \mu_{2}\right)$. Assume that the characters $\mu_{i}$ of $\mathbb{Q}_{p}$ are unramified. Then an Eisenstein series $E_{0}$ is defined with the data of $\Pi$ and $\eta$, so that $E^{\text {ord }}$ is exactly the ordinary projection of $E_{0}$ ( $E^{\text {ord }}=e E_{0}$, where $e$ is the map of ordinary projection). The only difference between $E_{0}$ considered in [ibid.] and $E^{\text {int }}$ in (31) is that local sections at $p$ are different. A special section at $p$ is taken to make 
sure the corresponding $E_{0}$ leads to the ordinary $p$-adic Eisenstein series with the optimal constant terms at cusps. But, for $E^{\text {int }}$, the local section at $p$ is spherical.

Define normalized actions of $U_{p}$ and $T_{p}$ operators ( $\| U_{p}$ and $\| T_{p}$ ) to a modular form on $\mathrm{U}\left(I_{\zeta}\right)$; refer to [Hsieh 2011a; 2011b].

Lemma 5.4. Let $E$ be a p-adic modular form of weight $(0, k), k>2$. Suppose that $E$ is unramified at $p$. Then we have

$E\left\|U_{p}\left(\alpha_{i}\right) \equiv E\right\| T_{p}\left(\alpha_{i}\right)(\bmod p)$,

$$
\text { for } \alpha_{i}=\left(\begin{array}{cc}
I_{6-i} & 0 \\
0 & p I_{i}
\end{array}\right) \in \mathrm{GL}_{6}\left(\mathbb{Q}_{p}\right) \simeq U\left(I_{\zeta}\right)\left(\mathbb{Q}_{p}\right), i=1,2, \ldots, 6 .
$$

The analogous result for modular forms on $\mathrm{U}(2,1)$ was due to Hida's observation. Hsieh summarized it in [2011a, Lemma 7.3], whose proof is essentially the same as that of Lemma 5.4. I want to emphasize that the formulas of the normalized Hecke operators at $p$ used in the proof are especially for holomorphic forms. So, we can safely apply this lemma to $E^{\text {int }}$ and $E^{\text {ord }}$.

Lemma 5.5. $E^{\text {ord }}=e E_{0}=C \cdot e E^{\text {int }}$, where $C$ is a $p$-adic unit.

Roughly speaking, the proof is as follows. Suppose the local sections at $p$ for the Eisenstein series $E^{\text {ord }}$ and $E^{\text {int }}$ are, respectively, $f_{p}^{\text {ord }}$ and $f_{p}^{\text {int }}$. There is a unique normalized ordinary local section at $p$, denoted by $f_{p}^{\text {ord }, N}$ (for the uniqueness, see [Hida 2004b; Hsieh 2011b, Remark 6.3]). Then

$$
f_{p}^{\text {ord }}=C_{1} f_{p}^{\text {ord }, N} \quad \text { and } \quad e f_{p}^{\text {int }}=C_{2} f_{p}^{\text {ord }, N} .
$$

Moreover it can be shown that $C=C_{1} C_{2}^{-1}$ is a $p$-adic unit. Combining the two lemmas above, we get

$$
E^{\text {ord }} \not \equiv 0\left(\bmod \mathfrak{m}_{p}\right) \Longleftrightarrow E^{\text {int }} \not \equiv 0\left(\bmod \mathfrak{m}_{p}\right) .
$$

We are left to show that $E^{\text {int }} \not \equiv 0\left(\bmod \mathfrak{m}_{p}\right)$.

From the calculation of Section 4, we can construct a linear functional on the space of holomorphic modular forms on $\mathrm{U}(3,1)$, such that

$$
l_{\theta}\left(E^{\mathrm{int}}\right)=\frac{\left\langle E_{\psi}^{\mathrm{int}}, \theta\right\rangle}{\langle\theta, \theta\rangle} \cdot \theta^{\beta}(1)
$$

And notice that $E_{\psi}^{\mathrm{int}}$ and $\theta$ are both in the space of holomorphic theta functions, and $\langle\cdot, \cdot\rangle$ is defined to be the inner product in this space. When $\theta$ is $\theta_{\varphi}$, we have $l_{\theta_{\varphi}}\left(E^{\text {int }}\right) \sim L^{\text {alg }}\left(\eta \chi^{-1}, l-\frac{1}{2}\right) L^{\text {alg }}\left(\eta \chi^{-1}, l-\frac{3}{2}\right) L^{\text {alg }}(\Pi, \chi, 1)$. Notice that $\chi$ is from the Weil representation and we can vary $\chi$ with only the restrictions that $\chi_{\infty}(z)=|z| / z$ and $\left.\chi\right|_{\mathbb{A}^{\times}}=\epsilon_{K / \mathbb{Q}} \cdot L^{\mathrm{alg}}\left(\eta \chi^{-1}, l-\frac{1}{2}\right)$ and $L^{\mathrm{alg}}\left(\eta \chi^{-1}, l-\frac{3}{2}\right)$ are both $p$-units for almost all $\chi$. These facts are due to Hida [2004a]. The question of whether $L^{\mathrm{alg}}(\Pi, \chi, 1)$ is a $p$-unit or not for infinitely many $\chi$ remains an open 
problem. Many results are known about nonvanishing modulo $p$ of special values of $L$-functions (refer to [Hida 2004a; Vatsal 2003] for results on $\mathrm{GL}_{1}$ and $\mathrm{GL}_{2} L$ functions). These facts suggest $L^{\mathrm{alg}}(\Pi, \chi, 1)$ may share the same property. Recent work of M. Brakočević on anticyclotomic $p$-adic $L$-functions of central critical Rankin-Selberg $L$-value may help prove the conjecture. I will address this question in my next paper.

From Appendix B, we see by choosing proper $\varphi$ that $\theta_{\varphi}$ is a $p$-integral theta function, and can serve as one element of the basis that spans the space of $p$-integral holomorphic theta functions $T\left(m, L, U_{f}\right)$. So $l_{\theta_{\varphi}}$ maps a $p$-integral modular form in $\mathrm{U}(3,1)$ to a $p$-adic integer.

From the discussion above, we have this:

Conjecture 5.6. $E^{\text {int }} \not \equiv 0\left(\bmod \mathfrak{m}_{\Lambda}\right)$.

Remark 5.7. As mentioned in the introduction, the computations explained in this paper can be generalized to an arbitrary nonquasisplit unitary group $\mathrm{U}(m, n)$ for $m>n$. Let $P=M N$ be the minimal parabolic subgroup of $\mathrm{U}(m, n)$, and $M=\mathrm{GL}_{n}(K) \times U(m-n)$. Given $\Phi \in I_{P}^{U(m, n)}(\Pi \otimes \eta($ det $\cdot), s)$, define the Eisenstein series $E(\Phi)$ and consider the Fourier-Jacobi coefficients $E_{\psi}(\Phi)$ as a theta function on the Jacobi group $\mathrm{U}(m-n) \cdot N$. Because $E(\Phi)$ can be written as the pullback of a Siegel-Eisenstein series $\mathbb{E}(\eta)$ on $\mathrm{U}(m, m)$, we can study $E_{\psi}(\Phi)$ using certain Fourier-Jacobi coefficients of $\mathbb{E}(\eta)$. So $E_{\psi}(\Phi)$ will have similar expressions as in Corollary 4.11. Again using the inner product of the space of theta functions on $\mathrm{U}(m-n) \cdot N$, we can define a linear functional on $E(\Phi)$ that leads to special $L$-values.

\section{Appendix A: Proof of the Siegel-Weil formula for $(U(2,2), U(1))$}

The Siegel-Weil formula for the dual reductive pair $(U(2,2), U(1))$ is formulated in Theorem 3.6. Let us first fix necessary notation and then give the proof.

Consider the dual reductive pair $(U(2,2), U(V))$, where $V$ is a Hermitian vector space of dimension 1 over $K$. Now $\mathrm{U}(2,2) \times U(V)$ acts on $S\left(V^{2}(\mathbb{A})\right)$ via the Weil representation $\omega_{\chi}$ determined by an additive character $\psi$ of $\mathbb{A}$ and a character $\chi$ of $\mathbb{A}_{K}^{\times} / K^{\times}$such that $\left.\chi\right|_{\mathbb{A}^{\times}}=\epsilon_{K / F}$. For $\phi \in S\left(V^{2}(\mathbb{A})\right)$, we have $f_{\phi, s} \in I_{\mathscr{P}^{\prime}}^{U(2,2)}(\chi, s)$, where $\mathscr{P}^{\prime}$ is the Siegel parabolic subgroup of $U(2,2)$ (refer to (17)). Theorem 3.6 states that the Eisenstein series $E\left(g, f_{\phi, s}\right)$ on $\mathrm{U}(2,2)$ is holomorphic at $s=-\frac{1}{2}$ and

$$
\left.E\left(g, f_{\phi, s}\right)\right|_{s=-\frac{1}{2}}=2 \int_{[U(V)]} \theta_{\phi}(g, u) d u .
$$

I give the proof of this theorem following the idea of Kudla and Rallis [1988], who proved the Siegel-Weil formula for $\left(\mathrm{Sp}_{n}, \mathrm{O}(V)\right)$ when $V$ is anisotropic. First, we prove that $E\left(g, f_{\phi, s}\right)$ is holomorphic at $s=-\frac{1}{2}$ by studying the analytic properties 
of the constant term. Denote $\int_{[U(V)]} \theta_{\phi}(g, u) d u$ by $I(g, \phi)$. Notice that $I(g, \phi)$ is orthogonal to all cusp forms on $\mathrm{U}(2,2)(\mathbb{A})$ and is moreover concentrated on the Borel subgroup $B$ in the sense that the constant term of $I(g, \phi)$ along any parabolic subgroup $P$ strictly containing $B$ is orthogonal to all cusp forms on the Levi factor of $P$. Since the same is true for $E\left(g, f_{\phi, s}\right)$, it suffices to show that the constant term of $E\left(g, f_{\phi, s}\right)$ along the Siegel parabolic subgroup at $s=-\frac{1}{2}$ is equal to that of $I(g, \phi)$.

Denote the constant term of $E\left(g, f_{\phi, s}\right)$ with respect to $\mathscr{P}^{\prime}$ by $E_{\mathscr{P}}\left(g, f_{\phi, s}\right)$. Then we have this:

Lemma A.1. For any $\mathscr{K}^{\prime}$-finite section $f, E\left(g, f_{s}\right)$ and $E_{\mathscr{P}^{\prime}}\left(g, f_{s}\right)$ have the same set of poles.

If $\phi$ is $\mathscr{K}^{\prime}$-finite, so is $f_{\phi}$. We can apply the lemma above to find the poles of $E\left(g, f_{\phi, s}\right)$. There are three terms in $E_{\mathscr{P}^{\prime}}\left(g, f_{\phi, s}\right)$, that is

$$
E_{\mathscr{P}^{\prime}}\left(g, f_{\phi, s}\right)=E_{\mathscr{P}^{\prime}}^{0}\left(g, f_{\phi, s}\right)+E_{\mathscr{P}^{\prime}}^{1}\left(g, f_{\phi, s}\right)+E_{\mathscr{P}^{\prime}}^{2}\left(g, f_{\phi, s}\right),
$$

corresponding to the Bruhat decomposition

$$
\mathrm{U}(2,2)(F)=\mathscr{P}^{\prime} \sqcup \mathscr{P}^{\prime} w_{13} \mathscr{P}^{\prime} \sqcup \mathscr{P}^{\prime} w_{2} \mathscr{P}^{\prime},
$$

where

$$
w_{13}=\left(\begin{array}{cc}
{ }^{1} & 1 \\
-1 &
\end{array}\right) \text { and } w_{2}=\left(\begin{array}{cc}
I_{2} \\
-I_{2}
\end{array}\right)
$$

We have $E_{\mathscr{P}^{\prime}}^{0}\left(g, f_{\phi, s}\right)=f_{\phi, s}(g)$. So $\left.E_{\mathscr{P}^{\prime}}^{0}\left(g, f_{\phi, s}\right)\right|_{s=-\frac{1}{2}}=\omega_{\chi}(g) \phi(0)$. The third term has

$$
E_{\mathscr{P}^{\prime}}^{2}\left(g, f_{\phi, s}\right)=\int_{\mathcal{N}^{\prime}(\mathbb{A})} f_{\phi, s}\left(w_{2} n g\right) d n=M(s) f_{\phi, s}(g),
$$

where $M(s)$ is the intertwining operator. The second term has

$$
E_{\mathscr{P}^{\prime}}^{1}\left(g, f_{\phi, s}\right)=\sum_{\gamma \in B_{1} \backslash \mathrm{GL}_{2}(K)} f_{\phi, s}^{1}(\gamma g),
$$

where $B_{1}$ is the Borel subgroup of $\mathrm{GL}_{2}(K)$ and

$$
f_{\phi, s}^{1}(\gamma g)=\int_{\mathcal{N}^{\prime \prime}(\mathbb{A})} f_{\phi, s}\left(w_{13} n g\right) d n,
$$

where $\mathcal{N}^{\prime \prime}$ is a subgroup of $\mathcal{N}^{\prime}$ such that for $n \in \mathcal{N}^{\prime \prime}$,

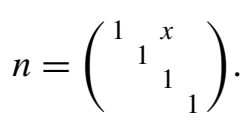


Since the Levi part of $\mathscr{P}^{\prime}(F)$ is isomorphic to $\mathrm{GL}_{2}(K)$, we see $\left.E_{\mathscr{P}^{\prime}}^{1}\left(g, f_{\phi, s}\right)\right|_{\mathrm{GL}_{2}\left(\mathrm{~A}_{K}\right)}$ is an Eisenstein series on $\mathrm{GL}_{2}\left(\mathbb{A}_{K}\right)$ associated to the section

$$
f_{\phi, s}^{1} \in I_{B^{1}}^{\mathrm{GL}_{2}}\left(\chi|\cdot|_{K}^{-s-\frac{1}{2}}, \chi|\cdot|_{K}^{s+\frac{3}{2}}\right),
$$

that is,

$$
f_{\phi, s}^{1}\left(\left(\begin{array}{rr}
a & b \\
& d
\end{array}\right) g\right)=\chi(a)|a|_{K}^{-s-\frac{1}{2}} \chi(b)|b|_{K}^{s+\frac{3}{2}}\left|\frac{a}{b}\right|_{K}^{\frac{1}{2}} f_{\phi, s}^{1}(g) .
$$

Let us consider the analytic property of $M(s) f_{\phi, s}(g)$. It is well known that $M(s)$ is well-defined for $s$ if $\operatorname{Re} s$ is big enough and it has the meromorphic continuation to the complex plane. Let $S$ be a finite set of places of $F$ such that $f_{\phi, s, v}=f_{0, s, v}$ is spherical for all $v \notin S$. Then we have

$$
M(s) f_{\phi, s}=\frac{a(s)}{b(s)} \prod_{v \in S} \frac{b(s)_{v}}{a(s)_{v}} M(s)_{v} f_{\phi, s, v} \prod_{v \notin S} f_{0,-s, v},
$$

where

$$
\frac{a(s)}{b(s)}=\frac{\xi(2 s-1,1) \xi\left(2 s, \epsilon_{K / F}\right)}{\xi(2 s+2,1) \xi\left(2 s+1, \epsilon_{K / F}\right)}
$$

specifically,

$$
\frac{a(s)_{v}}{b(s)_{v}}=\frac{L_{v}(2 s-1,1) L_{v}\left(2 s, \epsilon_{K / F}\right)}{L_{v}(2 s+2,1) L_{v}\left(2 s+1, \epsilon_{K / F}\right)}
$$

for $v \neq \infty$, and

$$
\frac{a(s)_{\infty}}{b(s)_{\infty}}=c(s) \frac{\Gamma(2 s-1) \Gamma(2 s+1)}{\Gamma(2 s+2) \Gamma(2 s+2)},
$$

where $c(s)$ is some exponential, which will not affect the analytic property of $M(s) f_{\phi, s}$.

Theorem A.2. $\left.M(s) f_{\phi, s}\right|_{s=-\frac{1}{2}}=0$

Proof. Notice that

$$
\left.\frac{a(s)}{b(s)}\right|_{s=-\frac{1}{2}}=0
$$

So we only need to show that

$$
\frac{b(s)_{v}}{a(s)_{v}} M(s)_{v} f_{\phi, s, v}
$$

for $v \in S$ at $s=-\frac{1}{2}$ is holomorphic.

When $v \in S$ and $v$ is a finite place, we always have this:

Lemma A.3. $\frac{1}{a(s)_{v}} M(s)_{v} f_{s, v}$ is holomorphic at $s=-\frac{1}{2}$ for any $f_{s}$. 
The general form of the lemma is stated in [Kudla and Rallis 1988]. If $v \in S$ is an inert place, $b(s)_{v}$ is obviously holomorphic at $s=-\frac{1}{2}$. So

$$
\frac{b(s)_{v}}{a(s)_{v}} M(s)_{v} f_{\phi, s, v}
$$

is holomorphic at $s=-\frac{1}{2}$.

If $v \in S$ is a splitting place, pay attention that now $b(s)_{v}$ has a simple pole. We need a refinement of Lemma A.3 as follows:

Lemma A.4. If $v$ is a splitting place,

$$
\frac{1}{a(s)_{v}} M(s)_{v} f_{\phi, s, v}
$$

vanishes at $s=-\frac{1}{2}$.

I have to emphasize that this lemma is only right for the Siegel-Weil section. Then in this case, we still have

$$
\frac{b(s)_{v}}{a(s)_{v}} M(s)_{v} f_{\phi, s, v}
$$

is holomorphic at $s=-\frac{1}{2}$. I omit the proof of Lemma A.4. It is a direct corollary of [Kudla and Sweet 1997, Theorem 1.3].

For $v=\infty$, for convenience, we take $\phi_{\infty}^{0}(x)=\psi\left(i x x^{*}\right)$ as it is what we use in the paper. For general functions in $S\left(V^{2}\right)_{\infty}$ in the space of the Fock representation, the holomorphic result still holds. We have that $\phi_{\infty}^{0}$ is an eigenfunction under the action of an element $k$ in the maximal open compact subgroup of $\mathrm{U}(2,2)_{\infty}$. Then, Lemma A.5. $M(s)_{\infty} f_{\phi_{\infty}^{0}, s}=c \frac{\Gamma(2 s) \Gamma(2 s-1)}{\Gamma\left(s+\frac{3}{2}\right) \Gamma\left(s+\frac{1}{2}\right)^{2} \Gamma\left(s-\frac{1}{2}\right)} f_{\phi_{\infty}^{0},-s}$.

From (35) and Lemma A.5, we can see that

$$
\frac{b(s)_{\infty}}{a(s)_{\infty}} M(s)_{\infty} f_{\phi_{\infty}^{0}, s}
$$

is holomorphic at $s=-\frac{1}{2}$. Combining the discussions above at each place, we prove Theorem A.2.

Now let us consider (33), which is the second term of the constant term of $E\left(g, f_{\phi, s}\right)$. As we see, restricted to the Levi part of $\mathrm{U}(2,2)$, it is an Eisenstein series associated to the (34). In [Kudla and Rallis 1988, Proposition 6.4], the Eisenstein series like this was discussed and the holomorphic property was confirmed. The idea is to obtain a relation of the form

$$
E_{\mathscr{P}^{\prime}}^{1}\left(g, f_{\phi, s}\right)=\sum_{j} \alpha_{j}(s) E^{1}\left(g, F_{j}(s)\right),
$$


where $\alpha_{j}(s)$ is a meromorphic function holomorphic at $s=-\frac{1}{2}$, and $E^{1}\left(g, F_{j}(s)\right)$ is an Eisenstein series on $\mathrm{GL}_{2}\left(\mathbb{A}_{K}\right)$ and

$$
F_{j}(g, s)=\mu_{1}(\operatorname{det} g)|\operatorname{det} g|_{K}^{\frac{1}{2}} \int_{\mathbb{A}_{K}^{\times}} \phi_{j}((0, x) g) \mu_{1} \mu_{2}^{-1}(\operatorname{det} g)|\operatorname{det} g|_{K} d x
$$

for $\phi_{j} \in S\left(M_{12}\left(\mathbb{A}_{K}\right)\right)$, and $\mu_{1}=\chi|\cdot|_{K}^{-s-\frac{1}{2}}, \mu_{2}=\chi|\cdot|_{K}^{s+\frac{3}{2}}$. The analytic property of this type of Eisenstein series is explicitly worked out. In our case, each $E^{1}\left(\cdot, F_{j}(s)\right)$ is holomorphic at $s=-\frac{1}{2}$. Furthermore, because the sum in (36) is a finite sum, $E_{\mathscr{P}^{\prime}}^{1}\left(g, f_{\phi, s}\right)$ is also holomorphic at $s=-\frac{1}{2}$.

Theorem A.6. $E\left(g, f_{\phi, s}\right)$ is holomorphic at $s=-\frac{1}{2}$.

It is not hard to verify that $I(g, \phi)$ as an automorphic form on $\mathrm{U}(2,2)$ is orthogonal to all cusp forms on $\mathrm{U}(2,2)$ and is concentrated on the Borel subgroup $B$. Since the constant term of $I(g, \phi)$ with respect to the Siegel parabolic subgroup $\mathscr{P}^{\prime}$ is $\omega_{\chi}(g) \phi(0)$, the only thing remaining to prove the Siegel-Weil formula is to confirm the constant term of $E\left(g, f_{\phi, s}\right)$ at $s=-\frac{1}{2}$ is $2 \omega_{\chi}(g) \phi(0)$.

Let us first calculate the constant term $E_{\mathscr{P}_{1}^{\prime}}\left(g, f_{\phi, s}\right)$ of $E\left(g, f_{\phi, s}\right)$ with respect to the parabolic subgroup $\mathscr{P}_{1}^{\prime}$ whose Levi factor is isomorphic to $K^{\times} \times U(1,1)$. Let

$$
i: U(1,1) \rightarrow U(2,2), \quad\left(\left(\begin{array}{ll}
a & b \\
c & d
\end{array}\right)\right) \mapsto\left(\begin{array}{lll}
1 & & \\
& a & b \\
& 1 & b
\end{array}\right) .
$$

Then:

Proposition A.7. For $g \in U(1,1)$,

$$
E_{\mathscr{P}_{1}^{\prime}}\left(g, f_{\phi, s}\right)=E_{\mathscr{P}_{1}^{\prime}}^{0}\left(g, s+\frac{1}{2}, i^{*} f_{\phi, s}\right)+E_{\mathscr{P}_{1}^{\prime}}^{1}\left(g, \frac{1}{2}-s, i^{*} M(s) f_{\phi, s}\right),
$$

where $E_{\mathscr{P}_{1}^{\prime}}^{0}$ and $E_{\mathscr{P}_{1}^{\prime}}^{1}$ are both Eisenstein series on $\mathrm{U}(1,1)$.

Notice that $E_{\mathscr{P}_{1}^{\prime}}^{0}$ is an Eisenstein series associated to the Siegel-Weil section $i^{*} f_{\phi, s}$. Applying the result about the Siegel-Weil formula for the dual reductive pair $(U(1,1), U(1))$ [Ichino 2004], we see that the constant term of

$$
E_{\mathscr{P}_{1}^{\prime}}^{0}\left(g, s+\frac{1}{2}, i^{*} f_{\phi, s}\right)
$$

with respect to the Siegel parabolic subgroup of $U(1,1)$ has two terms and they are both equal to $\omega_{\chi}(i(g)) \phi(0)$ for $g \in U(1,1)$ when $s=-\frac{1}{2}$.

First, $E_{\mathscr{O}_{1}^{\prime}}^{1}\left(g, \frac{1}{2}-s, i^{*} M(s) f_{\phi, s}\right)$ is holomorphic at $s=-\frac{1}{2}$ by arguments similar to those in [Kudla and Rallis 1988, Section 8]. Then applying Theorem A.2, we have this Eisenstein series is zero at $s=-\frac{1}{2}$. 
It seems that too many constant terms are involved. The relation can be described by the following diamond graph used in [ibid.]:

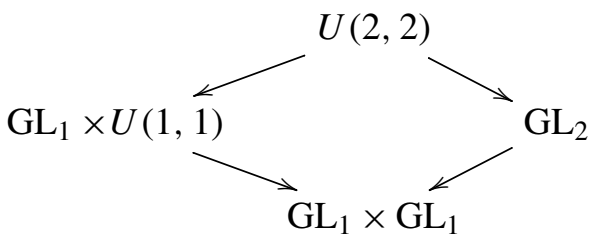

From the line

$$
\mathrm{U}(2,2) \rightarrow \mathrm{GL}_{1} \times U(1,1) \rightarrow \mathrm{GL}_{1} \times \mathrm{GL}_{1},
$$

we get $E_{\mathscr{P}_{1}^{\prime}}^{00}, E_{\mathscr{P}_{1}^{\prime}}^{01}, E_{\mathscr{P}_{1}^{\prime}}^{10}$, and $E_{\mathscr{P}_{1}^{\prime}}^{11}$. They are constant terms of $E_{\mathscr{P}_{1}^{\prime}}^{0}$ and $E_{\mathscr{P}_{1}^{\prime}}^{1}$. From the line

$$
\mathrm{U}(2,2) \rightarrow \mathrm{GL}_{2} \rightarrow \mathrm{GL}_{1} \times \mathrm{GL}_{1},
$$

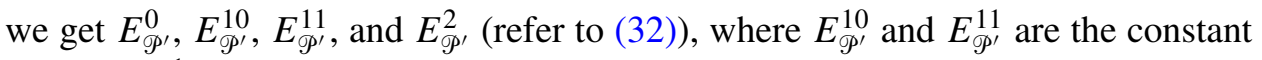
terms of $E_{\mathscr{P}^{\prime}}^{1}$. Restricting all these to $\mathrm{GL}_{1} \times \mathrm{GL}_{1}$, we have the following match up relation:

$$
\begin{array}{llll}
E_{\mathscr{P}_{1}^{\prime}}^{00} & E_{\mathscr{P}_{1}^{\prime}}^{01} & E_{\mathscr{P}^{\prime}}^{10} & E_{\mathscr{P}_{1}^{\prime}}^{11} \\
E_{\mathscr{P}^{\prime}}^{0} & E_{\mathscr{P}^{\prime}}^{10} & E_{\mathscr{P}^{\prime}}^{11} & E_{\mathscr{P}^{\prime}}^{2}
\end{array}
$$

The top terms match up with the bottom terms. Because $E_{\mathscr{P}_{1}^{\prime}}^{1}$ is zero at $s=-\frac{1}{2}$, then we have that $E_{\mathscr{P}^{\prime}}^{11}$ is zero at $s=-\frac{1}{2}$.

Proposition A.8. $\left.\quad E_{\mathscr{P}^{\prime}}^{1}\left(g, f_{\phi, s}\right)\right|_{s=-\frac{1}{2}}=\omega_{\chi}(g) \phi(0)$.

Thus, we obtain that $\left.E_{\mathscr{P}^{\prime}}\left(g, f_{\phi, s}\right)\right|_{s=-\frac{1}{2}}=2 \omega_{\chi}(g) \phi(0)$. Then Theorem 3.6 is proved.

\section{Appendix B: Integral theta functions}

First recall classical definitions of theta functions. For applications, let us restrict to the 2-dimensional case here. Most of the results can be generalized to arbitrary dimensions without difficulty. Fix the embeddings

$$
\overline{\mathbb{Q}} \stackrel{i_{\infty}}{\rightarrow} \mathbb{C} \quad \text { and } \quad \overline{\mathbb{Q}} \stackrel{i_{p}}{\rightarrow} \mathbb{C}_{p}
$$

Let $V$ be a 2-dimensional Hermitian vector space over $K$. Choose an $\mathscr{O}_{K}$-lattice $L$ so that it fixes an abelian variety $\mathscr{A}_{L}$ with complex multiplication defined over a number field $M$, where $K \subset M \subset \overline{\mathbb{Q}}$. Under the embedding $i_{\infty}, L$ can be regarded as a $\mathbb{Z}$-module of rank 4 , and so a lattice of $\mathbb{C}^{2}$. Then there exists an analytic parametrization over $\mathbb{C}$ such that

$$
\mathscr{A}_{L} \otimes_{i_{\infty}} \mathbb{C} \simeq \mathbb{C}^{2} / L
$$


For a Riemann form $H$ on $\mathbb{C}^{2} / L$, and a map $\epsilon: L \rightarrow U$, where $U$ is the unit circle of $\mathbb{C}$, define an analytic line bundle $\mathfrak{L}_{H, \epsilon}^{\mathrm{an}} \simeq \mathbb{C} \times \mathbb{C}^{2} / L$ with the action of $L$ given by

$$
l \cdot(w, x)=\left(w+l, \epsilon(l) e\left(\frac{1}{2 i} H\left(l, w+\frac{l}{2}\right)\right) x\right) \text { for } l \in L,(w, x) \in \mathbb{C}^{2} \times \mathbb{C},
$$

where $e(x)=e^{2 \pi i x}$. Then the space of global sections $\Gamma\left(\mathbb{C}^{2} / L, \mathfrak{L}_{H, \epsilon}^{\text {an }}\right)$ can be identified with the space of holomorphic theta functions $T(H, \epsilon, L)$ such that for $f \in T(H, \epsilon, L)$,

$$
f(w+l)=f(w) \epsilon(l) e\left(\frac{1}{2 i} H\left(l, w+\frac{l}{2}\right)\right) \text { for } w \in \mathbb{C}^{2}, l \in L .
$$

To study arithmetic theta functions and furthermore integral theta functions inside $T(H, \epsilon, L)$, let us consider the line bundle $\mathfrak{L}_{H, \epsilon}$ on $\mathscr{A}_{L}$ defined over $M$, and give $\mathfrak{L}_{H, \epsilon}$ a rigidification at the origin. At $\infty$, fix an isomorphism

$$
\mathfrak{L}_{H, \epsilon} \otimes_{i_{\infty}} \mathbb{C} \simeq \mathfrak{L}_{H, \epsilon}^{\mathrm{an}},
$$

such that it is consistent with the analytic parametrization of $\mathscr{A}_{L}$, and carries the rigidification of $\mathfrak{L}_{H, \epsilon}$ into the canonical one of $\mathfrak{L}_{H, \epsilon}^{a n}$. For a prime $p$, when $\mathscr{A}_{L} \otimes_{i_{p}} \mathbb{C}_{p}$ has a good reduction, we can assume it is defined over the ring of integers $\mathbb{O}=\mathbb{O}\left(\mathbb{C}_{p}\right)$. Then we require that the rigidification of $\mathfrak{L}_{H, \epsilon}$ satisfies that the $p$-integral elements of the stalk of $\mathfrak{L}_{H, \epsilon}$ over the origin correspond to the $p$-integral points on the affine line.

Within the context above, we see that $i_{\infty}\left(\Gamma\left(\mathscr{A}_{L}, \mathfrak{L}_{H, \epsilon}\right)\right)$ is an $i_{\infty}(M)$-vector space of theta functions inside $\Gamma\left(\mathbb{C}^{2} / L, \mathfrak{L}_{H, \epsilon}^{\mathrm{an}}\right)=T(H, \epsilon, L)$. Let us denote this space by $T^{\mathrm{ar}}(H, \epsilon, L)$; each element inside is an arithmetic theta function. Inside the $\mathbb{C}_{p}$-vector space $\Gamma\left(\mathscr{A}_{L} \otimes_{i_{p}} \mathbb{C}_{p}, \mathfrak{L}_{H, \epsilon} \otimes_{i_{p}} \mathbb{C}_{p}\right)$, we have the $\mathbb{O}$-module of integral sections. It is in turn the $i_{\infty}\left(i_{p}^{-1}(\mathcal{O}) \cap M\right)$-module of $p$-integral theta functions in $i_{\infty}\left(\Gamma\left(\mathscr{A}_{L}, \mathfrak{L}_{H, \epsilon}\right)\right)=T^{\mathrm{ar}}(H, \epsilon, L)$. We give it a notation $T^{\text {int }}(H, \epsilon, L)$. Thus we have

$$
T^{\text {int }}(H, \epsilon, L) \subset T^{\mathrm{ar}}(H, \epsilon, L) \subset T(H, \epsilon, L) .
$$

For $f \in T(H, \epsilon, L)$, define

$$
f_{*}(w)=e\left(\frac{i}{4} H(w, w)\right) f(w) .
$$

The following lemma gives simple characterizations of arithmetic theta functions and integral theta functions.

Lemma B.1. $T^{\mathrm{ar}}(H, \epsilon, L)$ consists of all functions $f \in T(H, \epsilon, L)$ such that $f_{*}(w)$ is an algebraic number in $\mathbb{C}$ for any $w \in K L$. The module $T^{\text {int }}(H, \epsilon, L)$ of p-integral theta functions consists of $f \in T(H, \epsilon, L)$ such that $i_{p} \cdot i_{\infty}^{-1}\left(f_{*}(w)\right)$ is integral in $\mathbb{C}_{p}$ for $w \in K L$. 
For the proof, see the proof [Finis 2006, Lemma 3.1]. Even though, there, only the case of one-dimensional Hermitian space was considered, the idea can be easily generalized here.

Shimura [1976] studied the arithmetic properties of theta functions with complex multiplication, and gave a basis for $T^{\mathrm{ar}}(H, \epsilon, L)$. Let me mainly discuss the case when $V / K$ is a 2-dimensional anisotropic Hermitian space. Also, $V$ can be regarded as a symplectic vector space with the alternating form

$$
\left\langle v_{1}, v_{2}\right\rangle=v_{1} \zeta v_{2}^{*}-v_{2} \zeta v_{1}^{*},
$$

with $v_{1} \zeta v_{2}^{*}$ the anti-Hermitian pairing on $V$. There exist $w_{1}, w_{2} \in M_{2}(K)$, such that $z=w_{2}^{-1} w_{1}$ is a point in the Siegel upper half plane $\mathfrak{H}_{2}$, and

$$
L=\left\{\left(\begin{array}{ll}
a & b
\end{array}\right)\left(\begin{array}{l}
w_{1} \\
w_{2}
\end{array}\right) \mid a \in \mathbb{Z}^{2}, b \in \mathbb{Z}^{2}\left(\begin{array}{ll}
1 & 0 \\
0 & \alpha
\end{array}\right) \text { for a fixed } \alpha \in \mathbb{Z}\right\} .
$$

Then the Riemann form on $V$ can be written as

$$
H\left(u w_{2}, v w_{2}\right)=2 i m \cdot u(z-\bar{z})^{-1} v^{*} \text { for } u, v \in K^{2},
$$

and $\epsilon$ is of the form

$$
\epsilon\left(a w_{1}+b w_{2}\right)=e\left(\frac{m}{2} \cdot a b^{t}+b r^{t}+a s^{t}\right) \quad \text { for } a \in \mathbb{Z}^{2}, b \in \mathbb{Z}^{2}\left(\begin{array}{ll}
1 & 0 \\
0 & \alpha
\end{array}\right),
$$

with a choice of $r$ and $s$ in $\mathbb{Q}^{2}$. For $u \in \mathbb{C}^{2}, z \in \mathfrak{H}_{2}, \quad r, s \in \mathbb{R}^{2}$, and a positive integer $\mu$, define

$$
\theta_{r, s}(u, z)=e\left(\frac{1}{2} u(z-\bar{z})^{-1} u^{t}\right) \sum_{x \in \mathbb{Z}^{2}} e\left(\frac{1}{2}(x+r) z(x+r)^{t}+(x+r)(u+s)^{t}\right) .
$$

Put $f_{m, r, s}(u)=\theta_{r, s}\left(m u w_{2}^{-1}, m w_{2}^{-1} w_{1}\right)$.

Theorem B.2. (1) For $r, s \in \mathbb{Q}^{2}$, we have $f_{m, r, s}(u) \in T^{\mathrm{ar}}(H, \epsilon, L)$ for $H$ defined in (38) and $\epsilon$ defined by

$$
\epsilon\left(a w_{1}+b w_{2}\right)=e\left(\frac{1}{2} m \cdot a b^{t}+m b r^{t}-a s^{t}\right) .
$$

Moreover, the functions

$$
f_{m, r+j, s} \quad \text { for } j \in m^{-1}\left(\begin{array}{cc}
1 & 0 \\
0 & \alpha^{-1}
\end{array}\right) \mathbb{Z}^{2} / \mathbb{Z}^{2}
$$

give a basis of $T^{\mathrm{ar}}(H, \epsilon, L)$ over the field of algebraic numbers in $\mathbb{C}$.

(2) Let $p$ be an unramified prime number for $T(H, \epsilon, L)$. When $z$ is diagonal, $f_{m, r, s}$ is a p-integral element in $T^{\mathrm{int}}(H, \epsilon, L)$. Thus $T^{\mathrm{int}}(H, \epsilon, L)$ is spanned by the functions $f_{m, r+j, s}$ over $i_{\infty}\left(i_{p}^{-1}(\mathcal{O})\right)$. 
Proof. See [Shimura 1976, Proposition 2.5] for the proof of (1). For (2), though it is possible to remove the condition that $z$ is diagonal, I will skip the discussion of the more general results because the proof would be more technical. When $z=\left(\begin{array}{cc}z_{1} & 0 \\ 0 & z_{2}\end{array}\right)$ is diagonal,

$$
\theta_{r, s}(u, z)=\prod_{i=1}^{2} \theta_{r_{i}, s_{i}}\left(u_{i}, z_{i}\right)
$$

where

$$
\theta_{r_{i}, s_{i}}\left(u_{i}, z_{i}\right)=e\left(\frac{1}{2} u_{i}\left(z_{i}-\bar{z}_{i}\right)^{-1} u_{i}\right) \sum_{x \in \mathbb{Z}} e\left(\frac{1}{2}\left(x+r_{i}\right) z_{i}\left(x+r_{i}\right)+\left(x+r_{i}\right)\left(u_{i}+s_{i}\right)\right),
$$

for $r=\left(r_{1}, r_{2}\right)$ and so on. Notice that these $\theta_{r_{i}, s_{i}}\left(u_{i}, z_{i}\right)$ are essentially theta functions in the one-dimensional case. The $p$-integrality of them is confirmed by [Finis 2006, Lemma 3.3]. Thus, the $p$-integrality of the $f_{m, r+j, s}$ follows.

Remark B.3. When $L$ corresponds to a diagonal element in $\mathfrak{H}_{2}, \mathscr{A}_{L}$ is isomorphic to the product of two elliptic curves. In this case, the $i_{\infty}\left(i_{p}^{-1}(\mathcal{O})\right)$-basis of $T^{\text {int }}(H, \epsilon, L)$ is given in Theorem B.2(2), and the number of elements inside is exactly equal to the dimension of $T^{\mathrm{ar}}(H, \epsilon, L)$. In general, when $\mathscr{A}_{L}$ is an abelian variety with $\mathrm{CM}$, it is always isogenous to $E_{1} \times E_{2}$, where $E_{1}$ and $E_{2}$ are elliptic curves with $\mathrm{CM}$. Such isogeny induces the isomorphism between modules of integral theta functions of $\mathscr{A}_{L}$ and $E_{1} \times E_{2}$ with appropriate choices of Riemann forms accordingly.

Then let me briefly explain the relation between classical theta functions and adelic theta functions. One can find the discussion of one-dimensional case in [Finis 2006]. Define $T_{\mathbb{A}}\left(m, L, U_{f}\right)$ to be the space of all smooth functions

$$
\Theta: N(\mathbb{Q}) U(\zeta)(\mathbb{Q}) \backslash N(\mathbb{A}) U(\zeta)(\mathbb{A}) / U(\zeta)_{\infty} U_{f} N(L)_{f} \rightarrow \mathbb{C}
$$

where $U_{f}$ is a certain open compact subgroup of $\mathrm{U}(\zeta)$ at finite places such that the level is prime to $p$, and

$$
N(L)_{f}=\left\{(w, t) \mid w \in \hat{L}, t+\frac{1}{2} w \zeta w^{*} \in \mu(L) \hat{\widehat{O}}_{K}\right\},
$$

where $\mu(L)$ is the ideal generated by $w \zeta w^{*}$ for all $w \in L$. The function $\Theta$ satisfies

$$
\Theta((0, t) r)=e(m t) \Theta(r) \quad \text { for } r \in N(\mathbb{A}) U(\zeta)(\mathbb{A}) .
$$

Because $\mathrm{U}(\zeta)$ is anisotropic, $\mathrm{U}(\zeta)(\mathbb{Q}) \backslash U(\zeta)(\mathbb{A}) / U(\zeta)_{\infty} U_{f}$ consists of a finite set of points $\left\{u_{1}, \ldots, u_{s}\right\} \subset U(\zeta)\left(\mathbb{A}_{f}\right)$. So we have

$$
T_{\AA}\left(m, L, U_{f}\right)=\bigoplus_{i=1}^{s} T\left(m, u_{i} L\right), \quad \Theta \mapsto\left(\Theta_{i}\right)
$$


such that $\Theta_{i}(n)=\Theta\left(n u_{i}\right)$ for $n \in N(\mathbb{A})$. Then one may check for each $i$,

$$
\theta_{i}\left(w_{\infty}\right)=e\left(-m \frac{w \zeta w^{*}}{2}\right) \Theta_{i}\left(\left(w_{\infty}, 0\right)\right)
$$

is a classical theta function in $T\left(H, \epsilon, u_{i} L\right)$, where $H$ and $\epsilon$ are defined according to the lattice $u_{i} L$, and

$$
\left\langle\Theta, \Theta^{\prime}\right\rangle=\sum_{i}\left\langle\theta_{i}, \theta_{i}^{\prime}\right\rangle \text { for } \Theta, \Theta^{\prime} \in T_{\AA}\left(m, L, U_{f}\right),
$$

where

$$
\left\langle\Theta, \Theta^{\prime}\right\rangle=\int_{N(\mathbb{Q}) U(\zeta)(\mathbb{Q}) \backslash N(A) U(\zeta)(\mathbb{A})} \Theta(r) \overline{\Theta^{\prime}(r)} d r
$$

and

$$
\left\langle\theta_{i}, \theta_{i}^{\prime}\right\rangle=\frac{1}{\mu(L)} \int_{\mathbb{C} / L} \theta_{i}(w) \overline{\theta_{i}^{\prime}(w)} d w
$$

\section{Acknowledgments}

This paper is revised from my thesis at Columbia University. I am deeply grateful to my advisor Eric Urban for the suggestion of studying this topic. His classes on Iwasawa theory and numerous discussions with him still serve as guidance for my research. I am also very thankful to many mathematicians from Columbia University, IAS, Princeton University, Northwestern University and others who share ideas with me and give me encouragement. Finally, I would like to thank the referee for suggestions that helped improve the paper.

\section{References}

[Finis 2006] T. Finis, "Divisibility of anticyclotomic $L$-functions and theta functions with complex multiplication", Ann. of Math. (2) 163:3 (2006), 767-807. MR 2007m:11151 Zbl 1111.11047

[Gelbart 1975] S. S. Gelbart, Automorphic forms on adèle groups, Annals of Mathematics Studies 83, Princeton University Press, 1975. MR 52 \#280 Zbl 0329.10018

[Godement and Jacquet 1972] R. Godement and H. Jacquet, Zeta functions of simple algebras, Lecture Notes in Mathematics 260, Springer, Berlin, 1972. MR 49 \#7241 Zbl 0244.12011

[Greenberg 1994] R. Greenberg, "Iwasawa theory and p-adic deformations of motives", pp. 193-223 in Motives (Seattle, WA, 1991), edited by U. Jannsen et al., Proc. Sympos. Pure Math. 55, Amer. Math. Soc., Providence, RI, 1994. MR 95i:11053 Zbl 0819.11046

[Harris et al. 1996] M. Harris, S. S. Kudla, and W. J. Sweet, "Theta dichotomy for unitary groups”, J. Amer. Math. Soc. 9:4 (1996), 941-1004. MR 96m:11041 Zbl 0870.11026

[Harris et al. 2006] M. Harris, J.-S. Li, and C. M. Skinner, " $p$-adic $L$-functions for unitary Shimura varieties, I: Construction of the Eisenstein measure", Doc. Math. Extra Vol. (2006), 393-464. MR 2008d:11042 Zbl 1143.11019

[Hida 2004a] H. Hida, "Non-vanishing modulo $p$ of Hecke $L$-values", pp. 735-784 in Geometric aspects of Dwork theory, vol. 2, edited by A. Adolphson et al., de Gruyter, Berlin, 2004. MR 2006b:11055 Zbl 1162.11342 
[Hida 2004b] H. Hida, p-adic automorphic forms on Shimura varieties, Springer, New York, 2004. MR 2005e:11054 Zbl 1055.11032

[Hsieh 2011a] M. Hsieh, "Eisenstein congruence on unitary groups and Iwasawa main conjecture for CM fields", preprint, 2011, available at http://www.math.ntu.edu.tw/ mlhsieh/research.htm.

[Hsieh 2011b] M.-L. Hsieh, "Ordinary $p$-adic Eisenstein series and $p$-adic $L$-functions for unitary groups”, Ann. Inst. Fourier (Grenoble) 61:3 (2011), 987-1059. MR 2918724 Zbl 06002990

[Ichino 2004] A. Ichino, "A regularized Siegel-Weil formula for unitary groups", Math. Z. 247:2 (2004), 241-277. MR 2005g:11082 Zbl 1071.11022

[Kudla and Rallis 1988] S. S. Kudla and S. Rallis, "On the Weil-Siegel formula", J. Reine Angew. Math. 387 (1988), 1-68. MR 90e:11059 Zbl 0644.10021

[Kudla and Sweet 1997] S. S. Kudla and W. J. Sweet, Jr., "Degenerate principal series representations for U(n,n)", Israel J. Math. 98 (1997), 253-306. MR 98h:22021 Zbl 0896.22007

[Li 1992] J.-S. Li, "Nonvanishing theorems for the cohomology of certain arithmetic quotients", $J$. Reine Angew. Math. 428 (1992), 177-217. MR 93e:11067 Zbl 0749.11032

[Mainardi 2004] F. Mainardi, Sur la conjecture principale de Iwasawa pour les corps CM, thesis, Université de Paris-Nord (Villetaneuse, Seine-Saint-Denis / Bobigny), 2004.

[Mazur and Wiles 1984] B. Mazur and A. Wiles, "Class fields of abelian extensions of Q", Invent. Math. 76:2 (1984), 179-330. MR 85m:11069 Zbl 0545.12005

[Murase and Sugano 2002] A. Murase and T. Sugano, "Fourier-Jacobi expansion of Eisenstein series on unitary groups of degree three", J. Mathematical Sciences Univ. Tokyo 9:2 (2002), 347-404. MR 2003f:11065 Zbl 1143.11319

[Ribet 1976] K. A. Ribet, "A modular construction of unramified $p$-extensions of $Q\left(\mu_{p}\right)$ ", Invent. Math. 34:3 (1976), 151-162. MR 54 \#7424 Zbl 0338.12003

[Shimura 1976] G. Shimura, "Theta functions with complex multiplication", Duke Math. J. 43:4 (1976), 673-696. MR 54 \#12664 Zbl 0371.14022

[Shimura 1997] G. Shimura, Euler products and Eisenstein series, CBMS Regional Conference Series in Mathematics 93, American Mathematical Society, Providence, RI, 1997. MR 98h:11057 Zbl 0906.11020

[Skinner and Urban 2012] C. Skinner and E. Urban, "The Iwasawa main conjecture for GL(2)", preprint, 2012, available at http://www.math.columbia.edu/ urban/eurp/MC.pdf.

[Urban 2001] E. Urban, "Selmer groups and the Eisenstein-Klingen ideal", Duke Math. J. 106:3 (2001), 485-525. MR 2002b:11073 Zbl 1061.11027

[Urban 2006] E. Urban, "Groupes de Selmer et fonctions $L p$-adiques pour les representations modulaires adjointes", preprint, 2006, available at http://www.math.columbia.edu/ urban/EURP.html.

[Vatsal 2003] V. Vatsal, "Special values of anticyclotomic L-functions", Duke Math. J. 116:2 (2003), 219-261. MR 2004e:11069b Zbl 0262.46032

[Weil 1965] A. Weil, "Sur la formule de Siegel dans la théorie des groupes classiques", Acta Math. 113 (1965), 1-87. MR 36 \#6421 Zbl 0161.02304

[Wiles 1990] A. Wiles, "The Iwasawa conjecture for totally real fields", Ann. of Math. (2) 131:3 (1990), 493-540. MR 91i:11163 Zbl 0719.11071

[Yang 1997] T. Yang, "Theta liftings and Hecke L-functions", J. Reine Angew. Math. 485 (1997), 25-53. MR 98e:11056 Zbl 0867.11037

Communicated by John $\mathrm{H}$. Coates

Received 2011-01-17 Revised 2012-02-03 Accepted 2012-03-03

zbtaishan@gmail.com Northwestern University, Department of Mathematics, 2033 Sheridan Rd., Evanston, IL 60202, United States 


\section{Algebra \& Number Theory}

msp.org/ant

\section{EDITORS}

MANAGING EDITOR

Bjorn Poonen

Massachusetts Institute of Technology

Cambridge, USA

\author{
EDITORIAL BOARD CHAIR \\ David Eisenbud \\ University of California \\ Berkeley, USA
}

\section{BOARD OF EDITORS}

Georgia Benkart

Dave Benson

Richard E. Borcherds

John H. Coates

J-L. Colliot-Thélène

Brian D. Conrad

Hélène Esnault

Hubert Flenner

Edward Frenkel

Andrew Granville

Joseph Gubeladze

Ehud Hrushovski

Craig Huneke

Mikhail Kapranov

Yujiro Kawamata

János Kollár

Yuri Manin

Barry Mazur

Philippe Michel
University of Wisconsin, Madison, USA

University of Aberdeen, Scotland

University of California, Berkeley, USA

University of Cambridge, UK

CNRS, Université Paris-Sud, France

University of Michigan, USA

Freie Universität Berlin, Germany

Ruhr-Universität, Germany

University of California, Berkeley, USA

Université de Montréal, Canada

San Francisco State University, USA

Hebrew University, Israel

University of Virginia, USA

Yale University, USA

University of Tokyo, Japan

Princeton University, USA

Northwestern University, USA

Harvard University, USA

École Polytechnique Fédérale de Lausanne
Susan Montgomery

Shigefumi Mori

Raman Parimala

Jonathan Pila

Victor Reiner

Karl Rubin

Peter Sarnak

Joseph H. Silverman

Michael Singer

Vasudevan Srinivas

J. Toby Stafford

Bernd Sturmfels

Richard Taylor

Ravi Vakil

Michel van den Bergh

Marie-France Vignéras

Kei-Ichi Watanabe

Efim Zelmanov
University of Southern California, USA

RIMS, Kyoto University, Japan

Emory University, USA

University of Oxford, UK

University of Minnesota, USA

University of California, Irvine, USA

Princeton University, USA

Brown University, USA

North Carolina State University, USA

Tata Inst. of Fund. Research, India

University of Michigan, USA

University of California, Berkeley, USA

Harvard University, USA

Stanford University, USA

Hasselt University, Belgium

Université Paris VII, France

Nihon University, Japan

University of California, San Diego, USA

\section{PRODUCTION}

production@msp.org

Silvio Levy, Scientific Editor

See inside back cover or msp.org/ant for submission instructions.

The subscription price for 2013 is US \$200/year for the electronic version, and \$350/year ( $\$ 40$, if shipping outside the US) for print and electronic. Subscriptions, requests for back issues and changes of subscribers address should be sent to MSP.

Algebra \& Number Theory (ISSN 1944-7833 electronic, 1937-0652 printed) at Mathematical Sciences Publishers, 798 Evans Hall \#3840, c/o University of California, Berkeley, CA 94720-3840 is published continuously online. Periodical rate postage paid at Berkeley, CA 94704, and additional mailing offices.

ANT peer review and production are managed by EditFLOW ${ }^{\circledR}$ from Mathematical Sciences Publishers.

\section{PUBLISHED BY}

mathematical sciences publishers

nonprofit scientific publishing

http://msp.org/

(C) 2013 Mathematical Sciences Publishers 


\section{Algebra \& Number Theory}

$\begin{array}{lll}\text { Volume } 7 & \text { No. } 2 & 2013\end{array}$

The system of representations of the Weil-Deligne group associated to an abelian variety 243 RUTGER NOOT

Fourier-Jacobi coefficients of Eisenstein series on unitary groups

BEI ZHANG

The phase limit set of a variety

MoUnIR NisSE and FRANK SoTTILE

Base change behavior of the relative canonical sheaf related to higher dimensional moduli

Zsolt PATAKFALVI

Two ways to degenerate the Jacobian are the same

JESSE LEO KASS

Arithmetic motivic Poincaré series of toric varieties

Helena Cobo Pablos and Pedro Daniel González Pérez

Maximal ideals and representations of twisted forms of algebras

Michael LAU and ARTURo PiAnZOLA

Higher Chow groups of varieties with group action 